Florida International University FIU Digital Commons

7-9-2008

\title{
Small sample confidence intervals for the mean of a positively skewed distribution
}

Cherylyn Almonte

Florida International University

DOI: $10.25148 /$ etd.FI13101599

Follow this and additional works at: https://digitalcommons.fiu.edu/etd

Part of the Statistics and Probability Commons

\section{Recommended Citation}

Almonte, Cherylyn, "Small sample confidence intervals for the mean of a positively skewed distribution" (2008). FIU Electronic Theses and Dissertations. 1074.

https://digitalcommons.fiu.edu/etd/1074

This work is brought to you for free and open access by the University Graduate School at FIU Digital Commons. It has been accepted for inclusion in FIU Electronic Theses and Dissertations by an authorized administrator of FIU Digital Commons. For more information, please contact dcc@fiu.edu. 


\section{FLORIDA INTERNATIONAL UNIVERSITY}

Miami, Florida

SMALL SAMPLE CONFIDENCE INTERVALS FOR THE MEAN OF A

POSITIVELY SKEWED DISTRIBUTION

A thesis submitted in partial fulfillment of the

requirements for the degree of

MASTER OF SCIENCE

in

STATISTICS

by

Cherylyn Almonte 
To: Dean Kenneth Furton

College of Arts and Sciences

This thesis, written by Cherylyn Almonte, and entitled Small Sample Confidence Intervals for the Mean of a Positively Skewed Distribution, having been approved in respect to style and intellectual content, is referred to you for judgment.

We have read this thesis and recommend that it be approved.

Zhenmin Chen

Sneh Gulati

Dinesh Sharma

Date of Defense: July 9, 2008

The thesis of Cherylyn Almonte is approved.

B. M. Golam Kibria, Major Professor

$\begin{gathered}\text { Dean Kenneth Furton } \\ \text { College of Arts and Sciences }\end{gathered}$
Dean George Walker
University Graduate School

Florida International University, 2008 


\section{DEDICATION}

To my mother, father, brothers and sisters whose faith, love and support made this thesis possible. 


\section{ACKNOWLEDGMENTS}

I have been very blessed to have so much support in writing my thesis. I want to thank everyone who took a part in it from the planning stages to the final draft.

Specifically, I would like to thank the members of my thesis committee, Dr. B. M. Golam Kibria, Dr. Zhenmin Chen, Dr. Sneh Gulati, and Dr. Denish Sharma for taking the time to serve on my thesis committee and for providing feedback after my presentation at the conference. I would also like to thank the Graduate School and the College of Arts and Sciences Dean's Office. Without their knowledge and support in the technical aspects of my thesis, this thesis would have never been completed so quickly. Most of all, I would like to thank my thesis advisor, Dr. B. M. Golam Kibria, who spent countless hours reading my numerous drafts and providing feedback on each one.

I would like to thank my family and friends for their understanding of all the time I spent devoted to my thesis which I know on numerous occasions they wished I could have devoted to them. I would like to thank my parents who provided everything I needed while in school and I would like to thank my brothers and sisters for understanding why I needed so much support. I would like to thank Jordan Smilovic who spent hours helping me revise my proposal. I would also like to give a special thanks to Jorge Jauregui who took the time to teach me how to run scripts and sacrificed his computer so I could run the simulation faster. 


\section{ABSTRACT OF THE THESIS \\ SMALL SAMPLE CONFIDENCE INTERVALS FOR THE MEAN OF A POSITIVELY SKEWED DISTRIBUTION}

by

Cherylyn Almonte

Florida International University, 2008

Miami, Florida

Professor B. M. Golam Kibria, Major Professor

This thesis proposes some confidence intervals for the mean of a positively skewed distribution. The following confidence intervals are considered: Student-t, Johnson-t, median-t, mad-t, bootstrap-t, BCA, $T_{1}, T_{3}$ and six new confidence intervals, the median bootstrap-t, mad bootstrap-t, median $T_{1}, \operatorname{mad} T_{1}$, median $T_{3}$ and the mad $T_{3}$. A simulation study has been conducted and average widths, coefficient of variation of widths, and coverage probabilities were recorded and compared across confidence intervals. To compare confidence intervals, the width and coverage probabilities were compared so that smaller widths indicated a better confidence interval when coverage probabilities were the same. Results showed that the median $T_{1}$ and median $T_{3}$ outperformed other confidence intervals in terms of coverage probability and the mad bootstrap- $t$, mad- $t$, and mad $T_{3}$ outperformed others in terms of width. Some real life data are considered to illustrate the findings of the thesis. 


\section{TABLE OF CONTENTS}

CHAPTER

1

2

2

\section{INTRODUCTION}

CONFIDENCE INTERVALS.

2.1 Introduction

2.2 Classical Approach.

2.2.1 Student- $t$.

2.2.2 Johnson- $t$.

2.2.3 Median- $t$.

2.2.4 Mad- $t$.

2.3 Bootstrap Approach

2.3.1 Bootstrap- $t$

2.3.2 Percentile Bootstrap.

2.3.3 Bias-Corrected Percentile Bootstrap....

2.3.4 Bias-Corrected Accelerated (BCA) Percentile Bootstrap. 1

2.4 Transformation Approach........................................... 1

2.4.1 Box-Cox Power Transformation.................................. 1

2.4.2 $\mathrm{T}_{1}$ and $\mathrm{T}_{2}$ Transformations................................. 1

2.4.3 $\mathrm{T}_{3}$ Transformation.................................................. 1

2.5 Proposed Confidence Intervals................................................... 1

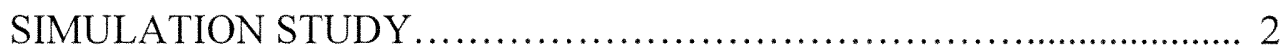

3.1 Introduction......................................................... 2

3.2 Simulation Technique............................................ 2

3.3 Results......................................................... 2

4

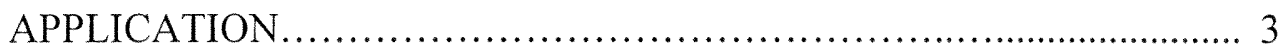

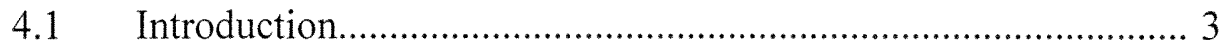

4.2 Age-Related Cataract Mortality ............................... 3

$4.3 \quad$ Psychotropic Drug Exposure........................................ 3

$4.4 \quad$ Mosquito Survival Rates............................................ 3

4.5 HIV-1 Prevalence................................................... 3

5 CONCLUDING REMARKS.......................................... 4

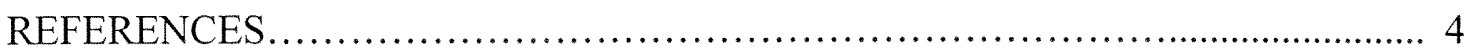

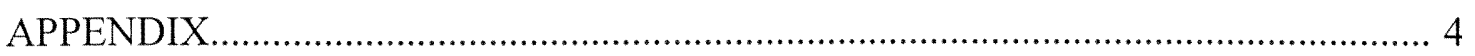




\section{LIST OF TABLES}

TABLE

$195 \%$ Confidence Intervals for Age-Related Cataract Mortality Data ............ 3

$295 \%$ Confidence Intervals for Psychotropic Drug Exposure Data ............... 3

$395 \%$ Confidence Intervals for Mosquito Survival Rates Data................. 3

$4 \quad 95 \%$ Confidence Intervals for HIV-1 Prevalence Data ........................ 4

A1 Estimated Coverage Probabilities using Gamma(16, .625) with

Skewness $=.5$.

A2 Estimated Coverage Probabilities using $\operatorname{Gamma}(4,2.5)$ with Skewness $=1 \ldots . .4$

A3 Estimated Coverage Probabilities using Gamma(1,10) with Skewness $=2 \ldots \ldots .4$

A4 Estimated Coverage Probabilities using $\operatorname{Gamma}(.25,40)$ with Skewness $=4 \ldots \quad 4$

A5 Estimated Coverage Probabilities using $\chi^{2}(32)$ with Skewness $=.5 \ldots \ldots \ldots \ldots .5$

A6 Estimated Coverage Probabilities using $\chi^{2}(8)$ with Skewness $=1 \ldots \ldots \ldots \ldots . \quad 5$

A7 Estimated Coverage Probabilities using $\chi^{2}(2)$ with Skewness $=2 \ldots \ldots \ldots \ldots \ldots . \quad 5$

A8 Estimated Coverage Probabilities using $\chi^{2}(.5)$ with Skewness $=4 \ldots \ldots \ldots \ldots .5$

A9 Estimated Coverage Probabilities using $\operatorname{Gamma}(4,2.5)$ with Skewness $=1$ including Median and Mad Bootstrap- $t$

A10 Estimated Coverage Probabilities using $\operatorname{Gamma}(.25,40)$ with Skewness $=4$

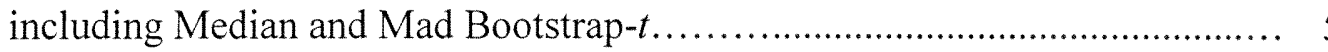

A11 Estimated Coverage Probabilities using $\log$ Normal $(2.25, .314)$ with Skewness $=1$ including Median and Mad Bootstrap- $t$

A12 Estimated Coverage Probabilities using Log Normal $(1.96, .833)$ with Skewness $=4$ including Median and Mad Bootstrap- $t$ 


\section{LIST OF FIGURES}

FIGURE

1 Confidence Coefficients for $\operatorname{Gamma}(4,2.5)$ with Skewness $=1$ including Median and Mad Bootstrap- $t$

2 Average Widths for $\operatorname{Gamma}(4,2.5)$ with Skewness $=1$ including Median and Mad Bootstrap- $t$

3 Confidence Coefficients for $\operatorname{Gamma}(.25,40)$ with Skewness $=4$ including Median and Mad Bootstrap- $t$.

4 Average Widths for $\operatorname{Gamma}(.25,40)$ with Skewness $=4$ including Median and Mad Bootstrap- $t$

$5 \quad$ Confidence Coefficients for Gamma with Various Skewness levels and Fixed Sample Size $(n=10)$.

6 Confidence Coefficients for Gamma with Various Skewness levels and Fixed Sample Size $(\mathrm{n}=50)$.

$7 \quad$ Histogram of Age-Related Cataract Mortality Data. 34

8 Histogram of Psychotropic Drug Exposure Data 36

9 Histogram of Mosquito Survival Rates Data. 38

10 Histogram of HIV-1 Prevalence Data 40 


\section{CHAPTER 1}

\section{INTRODUCTION}

Small samples with positively skewed distributions are common in the Health Sciences where experiments with rare diseases or atypical behaviors are the norm. These experiments typically involve significance tests which require a p-value to determine whether one should accept or reject the null hypothesis. Confidence intervals are an alternative to significance tests because they allow one to determine the significance of the test without finding a p-value. Additionally, they have a distinct advantage over significance tests because they give a measure of effect and a point of comparison between studies that a p-value is incapable of (Oakes, 1990; Rothman and Greenland, 1998; Visintainer and Tejani, 1998).

This thesis focuses on three approaches to construct a confidence interval for the mean of a skewed population: classical, bootstrap, and transformation. The classical approach is still the most widely used approach to construct confidence intervals. This approach includes the broadly used standard normal and Student- $t$ confidence intervals. Each confidence interval for the mean which uses the classical approach follows a similar pattern of estimating parameters from the sample and then adding and subtracting a factor based on a critical value and standard error. The bootstrap and transformation approaches, while not as widely used, are becoming more competitive against the classical approach because they may reduce error and have smaller widths. The bootstrap approach, unlike the classical approach, estimates its parameters from bootstrap samples which are created by resampling from the original sample normally at least 1000 to 2000 
times (Efron and Tibshirani, 1993). The transformation approach, similarly, does not use the original sample to estimate parameters. Instead, it transforms the original data so that the quantiles of this new data set can then be used to construct a confidence interval. These two approaches are not as widely used as the classical approach because they are computer intensive. However, with the increasing reliance on computers these approaches are becoming more competitive against the classical approach.

The classical Student- $t$ is the most widely used confidence interval because it is simple to calculate and it is robust for both small and large sample sizes. However when the population is positively skewed, the Student- $t$ will only have an approximate $1-\alpha$ coverage probability which may be improved by selecting a different confidence interval. This thesis reviews and proposes some confidence intervals using the classical, bootstrap, and transformation approaches which handle both small samples and positively skewed distributions. We consider the following confidence intervals: Student- $t$, Johnson- $t$, median- $t$, mad- $t$, bootstrap- $t$, bias-corrected accelerated (BCA) percentile bootstrap, $T_{1}$, $T_{3}$, and propose four new transformations methods: median $T_{1}$, median $T_{3}$, mad $T_{1}$ and mad $T_{3}$. Moreover, two additional confidence intervals: median bootstrap- $t$ and the mad bootstrap- $t$ have been proposed and briefly discussed. Since a theoretical comparison is not possible, a simulation study has been conducted and average widths, coefficient of variation, and coverage probabilities have been recorded and compared across confidence intervals. To compare confidence intervals, the width and coverage probabilities have been compared so that smaller widths indicate a better confidence interval when coverage 
probabilities are the same or higher coverage probabilities indicate a better confidence interval when widths are the same.

The objective of this research is two fold: first, to compare several interval estimators proposed by various researchers under the same simulation conditions and second, to propose some new methods and compare them with the existing methods. The organization of this thesis is as follows. The proposed confidence intervals have been given in Chapter 2. A Monte Carlo simulation study has been conducted in Chapter 3. As an application, some real life data have been analyzed in Chapter 4. Some concluding remarks are given in Chapter 5. 


\section{CHAPTER 2}

\section{CONFIDENCE INTERVALS}

\subsection{Introduction}

Let $X_{1}, X_{2}, \ldots, X_{n}$ be a random sample which is independently and identically distributed (i.i.d) and comes from a positively skewed distribution with unknown mean $\mu$ and unknown standard deviation $\sigma$. We want to find a $100(1-\alpha) \%$ confidence interval for $\mu$. Several methods for constructing this confidence interval have been discussed below.

\subsection{Classical Approach}

The classical approach is a well understood, simple, and widely used approach to construct confidence intervals. The traditional method for constructing a confidence interval for the mean using the classical approach is to estimate the parameters from the sample and then add and subtract a factor based on a critical value and standard error. The assumptions of each of these confidence intervals vary and though some of the confidence intervals in this approach rely on the normality assumption others do not. Additionally, some of these confidence intervals are intended for small samples and others are not. For example, the standard normal confidence interval is intended for large sample sizes whereas the well known Student- $t$ is intended for small sample sizes. In this section, we consider the following classical confidence intervals: Student- $t$, Johnson- $t$, median- $t$, and mad- $t$. 


\subsubsection{Student- $t$}

The Student- $t$ was developed by William Sealey Gosset (Student, 1908) as a more robust way of testing hypotheses specifically when sample sizes are below 30 and/or when the standard deviation $\sigma$ is unknown (Student, 1908). A 100(1- $\alpha) \%$ confidence interval for $\mu$ based on the Student- $t$ is given by

$$
\left[\bar{x} \pm t_{\alpha / 2, n-1} \frac{s}{\sqrt{n}}\right]
$$

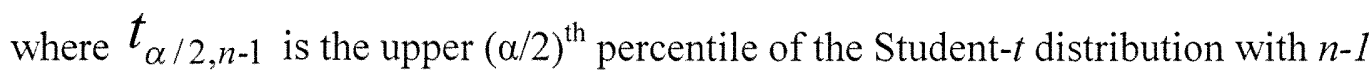
degrees of freedom and

$$
\bar{x}=\frac{\sum_{i=1}^{n} x_{i}}{n} \quad \text { and } \quad s=\sqrt{\frac{\sum_{i=1}^{n}\left(x_{i}-\bar{x}\right)^{2}}{n-1}}
$$

are the sample mean and standard deviation respectively. Since the Student- $t$ depends on the normality assumption, it may not be the best confidence interval for asymmetric distributions.

For this thesis, we assume that the random variable $\mathrm{X}$ follows a positively skewed distribution. Previous researchers have found that the Student- $t$ performs well for small samples sizes and asymmetric distributions in terms of the coverage probability coming close to the nominal confidence coefficient although its average widths and variability were not as small as other confidence intervals (Shi and Kibria, 2007; Wang, 2001; Zhou and Dinh, 2005). 


\subsubsection{Johnson- $t$}

An appropriate alternative to the Student- $t$ is the Johnson- $t$ which is a modification of the Student- $t$ that works well for asymmetric distributions. Using the Cornish-Fisher (1937) expansion, Johnson (1978) modified the Student- $t$ to allow for $\mu$ to be estimated from an asymmetric distribution. The Cornish-Fisher expansion relates the quantiles of the standard normal distribution to the population distribution using the sample moments of the population distribution. By using this expansion, the Johnson- $t$ is able to correct for the difference between the median and the mean which results from an asymmetric distribution (Johnson, 1978). Then following Johnson (1978), a 100(1- $\alpha) \%$ confidence interval for $\mu$ is given by

$$
\left[\bar{x}+\left(\frac{\hat{\mu}_{3}}{6 s^{2} n}\right)\right] \pm t_{\alpha / 2, n-1} \frac{s}{\sqrt{n}},
$$

where $t_{\alpha / 2, n-1}$ is the upper $(\alpha / 2)^{\text {th }}$ percentile of the Student- $t$ distribution with n-1 degrees of freedom, the estimate for the third central moment $\mu_{3}$ is

$$
\hat{\mu}_{3}=\frac{n \sum_{i=1}^{n}\left(x_{i}-\bar{x}\right)^{3}}{(n-1)(n-2)},
$$

and the sample variance is

$$
s^{2}=\frac{\sum_{i=1}^{n}\left(x_{i}-\bar{x}\right)^{2}}{n-1} .
$$

An advantage of the Johnson- $t$ is that it performs better than the Student- $t$ for asymmetric distributions in terms of having higher coverage probabilities and similar 
average widths and variations (Johnson, 1978; Shi and Kibria, 2007). The Johnson- $t$ can be a good replacement for the Student- $t$ however more simulation is needed to compare the Johnson- $t$ to the other confidence interval methods especially the new proposed intervals which may provide higher coverage probabilities and smaller widths.

\subsubsection{Median- $t$}

A less computationally intensive modification of the Student- $t$ was proposed by Shi and Kibria (2007). They proposed a new confidence interval called the median- $t$ which uses a modification of the standard deviation calculated using the deviations from the median of the distribution rather than the mean. A $100(1-\alpha) \%$ confidence interval for $\mu$ is given as follows

$$
\left[\bar{x} \pm t_{\alpha / 2, n-1} \frac{\tilde{s}}{\sqrt{n}}\right]
$$

where $t_{\alpha / 2, n-1}$ is the upper $(\alpha / 2)^{\text {th }}$ percentile of the Student- $t$ distribution with n-1 degrees of freedom, the sample variation is

$$
\tilde{S}=\sqrt{\frac{\sum_{i=1}^{n}\left(x_{i}-\tilde{x}\right)^{2}}{n-1}}
$$

and the sample median is $\tilde{x}$.

The median- $t$ is computationally simpler than the Johnson- $t$ and therefore analytically a more desirable method. For highly skewed distributions, Shi and Kibria (2007) found that the median- $t$ has better coverage probabilities in comparison to the Johnson- $t$ and Student- $t$ but it produces slightly wider widths and therefore more 
variation. Another measure they used was the coverage to width ratio which they defined as the confidence coefficient divided by the average width. For this measure, a higher coverage to width ratio indicates a better confidence interval. The coverage to width ratio of the median- $t$ in comparison to the Student- $t$ is slightly lower and presumably these results would apply to the Johnson- $t$ as well (Shi and Kibria, 2007).

Overall, the median- $t$ improves the Student- $t$ in terms of higher coverage probabilities. However, the median- $t$ is not accurate since the median- $t$ uses the $t$ distribution to calculate its critical value even though the distribution of the statistic may not be $t$. The distribution deviates from $t$ because the median is used to calculate the standard deviation instead of the mean. Following the median method proposed by Shi and Kibria (2007), we propose the following intervals: median bootstrap-t, median $T_{1}$, and median $T_{3}$, which do not depend on the normality assumption.

\subsubsection{Mad- $t$}

Shi and Kibria (2007) also proposed a confidence interval called the mad- $t$ which is calculated using the sample mean absolute deviation (MAD) instead of sample standard deviation. Following this idea, a $100(1-\alpha) \%$ confidence interval for $\mu$ is defined as

$$
\left[\bar{x} \pm t_{\alpha / 2, n-1} \frac{\hat{s}}{\sqrt{n}}\right]
$$

where $t_{\alpha / 2, n-1}$ is the upper $(\alpha / 2)^{\text {th }}$ percentile of the Student- $t$ distribution with n-1 degrees of freedom and the sample mean absolute deviation is 


$$
\hat{s}=\frac{1}{n} \sum_{i=1}^{n}\left|x_{i}-\bar{x}\right|
$$

Like the median- $t$, the mad- $t$ is computationally simpler than the Johnson- $t$ and therefore analytically a more desirable method. Shi and Kibria (2007) showed the mad- $t$ has a smaller average width than the Student- $t$, Johnson- $t$, and median- $t$. Additionally, they showed that the mad- $t$ has a higher coverage to width ratio than the Student-t, Johnson- $t$ and median- $t$. However, coverage probabilities for the mad- $t$ are constant and consistently lower than Student- $t$, Johnson- $t$, and median- $t$ for all sample sizes and from slightly to moderately skewed distributions (Shi and Kibria, 2007). These results follow the typical inverse relationship between width and coverage probability.

Overall, the mad- $t$ improves the Student- $t$ in terms of width. However, both median- $t$ and mad- $t$ used the percentile points from the $t$ distribution when the distribution of the statistic may not be $t$. Therefore, the results using this confidence interval may not be very accurate. We followed the mad method proposed by Shi and Kibria (2007) to propose three additional confidence intervals which do not depend on the normality assumption: mad bootstrap- $t$, mad $T_{1}$, and mad $T_{3}$.

\subsection{Bootstrap Approach}

The bootstrap approach (Efron, 1979) is an alternative to the classical approach. Though it is computer intensive, it is likely that it will have better coverage probabilities and reduce error for confidence intervals that have samples from skewed populations. The bootstrap approach is mostly used when there is no information about the underlying distribution-- although some parametric bootstrap confidence intervals exist. 
We can find a bootstrap sample by randomly sampling the data points, $\mathrm{n}$ times, with replacement from the same sample. We can denote this new sample as $X^{(*)}=X^{(*)}, X^{(*)}, \ldots \ldots \ldots X^{(*)}{ }_{n}$ where the $\mathrm{i}^{\text {th }}$ sample is denoted $\mathrm{X}^{(\mathrm{i})}$ for $\mathrm{i}=1,2, \ldots \mathrm{B}$, and $\mathrm{B}$ is the number of bootstrap samples. We want B to be as small as possible so that the computations are faster. However, Efron (1987) showed reducing B to a value as small as 400 causes the conditional coefficient of variation to become too large so he recommended the bootstrap sample to be at least 1000 . Therefore, the number of bootstrap samples (B) is typically between 1000 and 2000; because, the accuracy of the confidence interval depends on the size of the samples (Efron and Tibshirani, 1993). In this section, we consider the bootstrap- $t$, percentile bootstrap, bias-corrected percentile bootstrap and the bias-corrected accelerated (BCA) percentile bootstrap.

\subsubsection{Bootstrap- $t$}

Efron (1982) introduced the bootstrap- $t$ which used standardized $t$ scores to find the critical values for the confidence interval. To use this method, we first calculate standardized $t$ scores for each of the bootstrap samples using the formula

$$
T^{(i)}=\frac{\bar{x}^{(i)}-\bar{x}}{\sqrt{\frac{1}{(B-1)} \sum_{i=1}^{B}\left(\bar{x}^{(i)}-\bar{x}\right)^{2}}}
$$

where the mean of each $X^{(*)}$ is defined as

$$
\bar{x}^{(i)}=\frac{\sum_{j=1}^{n} x_{j}^{(i)}}{n}, \mathrm{i}=1,2,3, \ldots \mathrm{B} .
$$


We then order the $T^{(\mathrm{i})}$, s from smallest to largest to find $T_{(\alpha / 2)}$ and $T_{(1-\alpha / 2)}$ which are the $(\alpha / 2)^{\text {th }}$ and $(1-\alpha / 2)^{\text {th }}$ sample quantiles of the $T^{(\mathrm{i})}$ 's. Then, a $100(1-\alpha) \%$ confidence interval for $\mu$ is defined as follows:

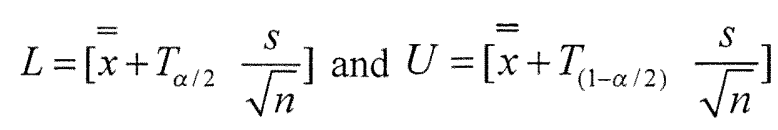

where $\mathrm{L}$ is the lower limit, $\mathrm{U}$ is the upper limit, $\mathrm{s}$ is the sample standard deviation, and the overall mean is

$$
\bar{x}=\frac{\sum_{i=1}^{B} \bar{x}^{(i)}}{B} .
$$

The bootstrap-t performs well in terms of having smaller average widths and better coverage to width ratios than the Student- $t$, median- $t$, and mad- $t$; however, it has consistently lower coverage probabilities than the three (Shi and Kibria, 2007). Again, these results follow the typical inverse relationship between width and coverage probability.

\subsubsection{Percentile Bootstrap}

Another bootstrap method introduced by Efron (1979) is the percentile bootstrap. With this approach, no information is needed about the underlying distribution of the sample. To construct this confidence interval, we calculate the sample mean of each of the $\mathrm{X}^{(*)}$ as before. Then, we order these means from smallest to largest and form a $100(1-\alpha) \%$ confidence interval for $\mu$ as follows,

$$
L=\bar{x}_{\left[(\alpha / 2)^{*} B\right]} \text { and } U=\bar{x}_{\left[(1-\alpha / 2)^{*} B\right]} \text {. }
$$


Shi and Kibria (2007) found the percentile bootstrap performs worse than the mad-t, median- $t$ and Student- $t$ in terms of coverage probabilities and lower coverage to width ratio. Moreover, the percentile bootstrap was later improved by Efron (1982). Therefore, we have not considered the percentile bootstrap in this thesis.

\subsubsection{Bias-Corrected Percentile Bootstrap}

Efron (1982) suggested a bias-corrected percentile bootstrap in order to correct for bias in the percentile bootstrap when the distribution is asymmetric. To use this method, we need to calculate the lower and upper percentile endpoints from the standard normal distribution using the formulas

$$
L=\Phi\left(2 Z_{P}+Z_{\alpha / 2}\right) \text { and } U=\Phi\left(2 Z_{P}+Z_{(1-(\alpha / 2))}\right)
$$

where $Z_{P}$ can be found by first finding an $i$ that would make the estimate of $\mu$ for the original sample data fall between

$$
\bar{x}_{[i] \text { and }} \bar{x}_{[i+1]} \text {, }
$$

then using this $\mathrm{i}$ to determine $\mathrm{P}=\mathrm{i} / \mathrm{B}$, and finally solving for $\mathrm{Z}_{\mathrm{p}}$ using the formula

$$
Z_{P}=\Phi^{-1}(P) \text {. }
$$

Then, a 100(1- $\alpha) \%$ confidence interval for $\mu$ can be found using the following formula

$$
\left(\bar{x}_{[L \times B]} \leq \mu \leq \bar{x}_{[U \times B]}\right) \text {. }
$$

Again, this method was later improved by Efron (1987) and therefore this bootstrap is not considered in this thesis. 


\subsubsection{Bias-Corrected Accelerated (BCA) Percentile Bootstrap}

To improve the bias-corrected percentile bootstrap, Efron (1987) introduced the bias-corrected accelerated (BCA) percentile bootstrap. This confidence interval corrects for bias when the distribution is asymmetric as well as the acceleration for the change in variation. To construct this confidence interval, first calculate the percentile endpoints form the standard normal distribution using the formulas

$$
L_{A}=\Phi\left(Z_{P}+\frac{Z_{P}+Z_{\alpha / 2}}{1-a\left(Z_{P}+Z_{\alpha / 2}\right)}\right) \text { and } U_{A}=\Phi\left(Z_{P}+\frac{Z_{P}+Z_{(1-(\alpha / 2))}}{1-a\left(Z_{P}+Z_{(1-(\alpha / 2))}\right)}\right)
$$

where $Z_{\mathrm{p}}$ is found as before and the acceleration constant is defined as

$$
a=\frac{\sum_{i=1}^{n}\left(\bar{x}^{(\cdot)}-\dot{\bar{x}}^{(i)}\right)^{3}}{6\left[\sum_{i=1}^{n}\left(x^{(\cdot)}-\bar{x}\right)^{2}\right]^{3 / 2}},
$$

where

$$
=\frac{\sum_{i=1}^{n} \dot{-(i)}}{n} \text {, }
$$

and $\dot{\bar{x}}^{(i)}$ is computed from the original sample with the $\mathrm{i}^{\text {th }}$ point deleted. Then, a $100(1-$ $\alpha) \%$ confidence interval for $\mu$ is given by

$$
\left(\bar{x}_{\left[L_{A} \times B\right]} \leq \mu \leq \bar{x}_{\left[U_{A} \times B\right]}\right) .
$$

Finding a good estimate for the acceleration constant can be very difficult and there are numerous methods for finding it; thus, this confidence interval method may not be the best method because of the extensive computations required to find the acceleration constant that gives the most accurate confidence interval (Shao and $\mathrm{Tu}$, 
1995). However, an advantage of the BCA is that it performs substantially better than the

Student- $t$ in terms of estimated width and variability although it was not checked against any of the other previously discussed methods (Wang, 2001). Zhou and Dinh (2005) found that the BCA performed better than the bootstrap- $t$ and the Student- $t$ in terms of widths; however, the coverage probabilities were about the same as the Student- $t$ and much lower than the bootstrap- $t$. It is unclear whether the bootstrap- $t$ performs better than the BCA overall and thus more simulation is needed.

\subsection{Transformation Approach}

An alternative to the bootstrap and classical approaches is the transformation approach. Sometimes, a transformation of the data will help to create a confidence interval which has coverage probabilities close to the nominal values. In these cases, a transformation approach might be more appropriate and exact than approximation using the original asymmetric distribution. To construct these confidence intervals, the original data is transformed so that the quantiles of the transformed data can then be used to construct the confidence interval. This approach is computer intensive, like the bootstrap approach, however with the increasing reliance on computers it is becoming more competitive against the classical approach. Additionally, many transformation confidence intervals can be computed faster than competing bootstrap confidence intervals. This thesis discusses the following transformation confidence intervals: Box-Cox power transformation $T_{1}, T_{2}$, and $T_{3}$. 


\subsubsection{Box-Cox power Transformation}

A popular transformation method named the Box-Cox power transformation was introduced by Box and Cox (1964). This transformation provides a formula for a family of distributions so that when the appropriate exponent is selected the data will be transformed and the skewness will be removed. The formula to transform $\mathrm{X}$ is given by

$$
f(x ; \lambda)=\left\{\begin{array}{lll}
\frac{\left(x^{\lambda}-1\right)}{\lambda} & \text { if } & \lambda \neq 0 \\
\ln (x) & \text { if } & \lambda=0
\end{array}\right.
$$

where the maximum likelihood estimate of $\lambda$ is given by

$$
x_{i(\lambda)}=\left\{\begin{array}{ll}
\frac{x_{i}^{\lambda}-1}{\lambda \bar{x}^{(\lambda-1)}} & \text { if } \lambda \neq 0 \\
\bar{x} \ln \left(x_{i}\right) & \text { if } \lambda=0
\end{array} .\right.
$$

To transform the data correctly we should have all $x_{i}>0$, for $i=1,2,3 \ldots$. n. Box and Cox (1964) suggested using the maximum likelihood estimate of $\lambda$ which makes the transformed data approximately normal. Box and Cox showed that the maximum likelihood estimator of $\lambda$ is the value of $\lambda$ which minimizes the residual sum of squares of the fitted model. Therefore, we want to minimize

$$
S=\frac{\sum_{i=1}^{n}\left(x_{i(\lambda)}-\bar{x}_{(\lambda)}\right)^{2}}{n-1}
$$

where

$$
\bar{x}_{(\lambda)}=\frac{\sum_{i=1}^{n} x_{i(\lambda)}}{n} .
$$


Then, a $100(1-\alpha) \%$ confidence interval for $\mu$ can then be obtained only after some extensive computations. For details about the Box-Cox power transformation, we refer the reader to Wang (2001). In general, his simulation results showed that the bootstrap method is more effective and efficient than the Box-Cox power transformation, standard normal, and Student- $t$ methods. His results also indicated that the Box-Cox power transformation had low coverage probabilities compared to the Student- $t$ and BCA; however, it also had smaller average widths and variability than the Student- $t$ but not the $\mathrm{BCA}$. Thus, the BCA was overall a better approach than transforming the data.

\subsection{2 $T_{1}$ and $T_{2}$ Transformations}

Two alternative transformations, the $T_{1}$ and $T_{2}$ transformations, are introduced by Hall (1992). These transformations are based on the Edgeworth expansion which is similar to the Cornish-Fisher expansion because they both relate one probability distribution function to another. Using the Edgeworth expansion, these transformations correct for both the bias and skewness of asymmetric distributions. Details about the methods to obtain these transformations and the resulting formulas can be found in Hall (1992). Zhou and Dinh (2005) simplified these transformations and defined them by their inverses

$$
\begin{aligned}
& T_{1}^{-1}(t)=\left(\frac{3}{\hat{\gamma}}\right)\left[1+\hat{\gamma}\left(t-\left(\frac{\hat{\gamma}}{6 n}\right)\right)\right]^{\frac{1}{3}}-\left(\frac{3}{\hat{\gamma}}\right) \\
& T_{2}^{-1}(t)=\left[\frac{3 \sqrt{n}}{2 \hat{\gamma}}\right] \log \left[\frac{2 \hat{\gamma}}{3 \sqrt{n}}\left(t-\frac{\hat{\gamma}}{6 n}\right)+1\right]
\end{aligned}
$$


where $\hat{\gamma}=\frac{\sum_{i=1}^{n}\left(x_{i}-\bar{x}\right)^{3}}{s^{3}}$ is the estimated population skewness. Then, a $100(1-\alpha) \%$

confidence interval for $\mu$ is obtained as

$$
\begin{aligned}
& L=\bar{X}-T_{i}^{-1}\left(\frac{\phi(1-(\alpha / 2))}{\sqrt{n}}\right) s \\
& U=\bar{X}-T_{i}^{-1}\left(\frac{\phi(\alpha / 2)}{\sqrt{n}}\right) s
\end{aligned}
$$

where $s$ is the sample standard deviation and $\phi()$ refers to the quantiles of the Standard Normal.

Hall (1992) found that both $T_{1}$ and $T_{2}$ have better coverage probabilities than the Student- $t$, Johnson- $t$, and Cornish-Fisher. There was no clear indication as to whether $T_{1}$ performed better than $T_{2}$ or vice versa. Zhou and Dinh (2005), however, found that $T_{1}$ performed better than $T_{2}$ in terms of coverage probabilities and its average widths were about the same. For this reason, we consider only the $T_{1}$ transformation.

\subsection{3 $T_{3}$ Transformation}

Zhou and Dinh (2005) continued on Hall's transformations and proposed a new transformation. This transformation, the $T_{3}$, is computationally simpler than the previous two. The original formula can be defined by its inverse as follows:

$$
T_{3}^{-1}(t)=\left[1+3\left(t-\left(\frac{\hat{\gamma}}{6 n}\right)\right)\right]^{\frac{1}{3}}-1
$$


Then, one can construct a $100(1-\alpha) \%$ for $\mu$ as follows

$$
\begin{aligned}
& L=\bar{X}-T_{3}^{-1}\left(\frac{\phi(1-(\alpha / 2))}{\sqrt{n}}\right) s \\
& U=\bar{X}-T_{3}^{-1}\left(\frac{\phi(\alpha / 2)}{\sqrt{n}}\right) s
\end{aligned}
$$

where $\mathrm{s}$ is the sample standard deviation and $\phi($.$) refers to the quantiles of the standard$ normal. Zhou and Dinh (2005) found that the $T_{3}$ transformation was performing about the same as the bootstrap- $t$ and $T_{1}$ transformation in terms of both coverage probability and width. For this reason, the bootstrap-t, $T_{1}$ transformation, and $T_{3}$ transformation have all been considered.

\subsection{Proposed Confidence Intervals}

The confidence intervals from previous research selected for simulation are as follows: Student- $t$, Johnson- $t$, median- $t$, mad- $t$, bootstrap- $t$, BCA, $T_{1}$ transformation, and $T_{3}$ transformation. These confidence intervals have shown their merits by various researchers in different times but have not been compared at the same time under the same simulation conditions.

In addition to studying the selected confidence intervals, six new confidence intervals have been proposed. These confidence intervals are based on the median- $t$ and mad- $t$ confidence intervals proposed by Shi and Kibria (2007). To construct the median- $t$ and mad- $t$ confidence intervals, Shi and Kibria (2007) used percentile points from the Student $t$ distribution to calculate critical values (see section 2.2.3 and 2.2.4). However, 
when one replaces the standard deviation s by $\tilde{s}$ (as in section 2.2.3) or by $\bar{s}$ (as in section 2.2.4), in the denominator of the Student's $t$ distribution, there is no guarantee that the test statistic will follow a Student's $t$ distribution. Even though they have shown that their proposed intervals work well compared to others, the new proposed confidence intervals based on the bootstrap sample are more logical than the median- $t$ and mad- $t$ because they do not rely on the $t$ distribution. In this thesis, we proposed the following confidence intervals: the median bootstrap-t, the median $T_{1}$ and the median $T_{3}$, which are modifications of the median- $t, T_{1}$ transformation, and $T_{3}$ transformation respectively. Similarly, we proposed the mad versions of these confidence intervals: mad bootstrap-t, $\operatorname{mad} T_{1}$ transformation, and $\operatorname{mad} T_{3}$ transformation.

Since a theoretical comparison is not feasible, a Monte Carlo simulation study has been conducted to compare the performance of the proposed intervals in the following Chapter. 


\section{CHAPTER 3}

\section{SIMULATION STUDY}

\subsection{Introduction}

Since a theoretical comparison is difficult, following Shi and Kibria (2007) a simulation study has been conducted to compare the performance of the confidence intervals. Based on the results of the simulation study, the best confidence interval will be chosen based on average width, coefficient of variation of the widths, coverage probability, sample size and skewness level. The program for the simulation has been conducted using S-plus 8.0. The procedures have been described in section 3.2. The results of the simulation have been discussed in section 3.3. To generate data, we consider the gamma, chi-square, and log normal distributions with various skewness levels. We want to find some good confidence intervals which will be useful for a small sample coming from a positively skewed distribution.

\subsection{Simulation Technique}

The flowchart for this design is as follows:

i) Select the sample size (n), number of simulation runs (M), number of bootstrap samples (B), and significance level $(\alpha)$.

ii) Generate a sample from the gamma, chi-square, or log normal distribution with the chosen population skewness. 
iii) Construct the confidence intervals at a 1- $\alpha$ confidence level using the formulas defined in Chapter 2 where the bootstrap samples are created by resampling from the original sample B times with replacement.

iv) For each confidence interval constructed, determine if the confidence interval includes the population mean $\mu$ and for those confidence intervals that contain the mean record the width.

(v) Repeat (i)-(iv) M times. Then, compute the coverage probability (CP) (the proportion of intervals that contain the true mean out of $M$ intervals), the average width and the coefficient of variations (CV) of the widths.

The parameters for the gamma distribution have been chosen following Shi and Kibria (2007); the sample $X_{1}, X_{2}, \ldots \ldots . . X_{n}$ was taken from the following gamma distributions with a common mean 10 : $\mathrm{G}(16,625)$ with skewness $.5, \mathrm{G}(4,2.5)$ with skewness $1, \mathrm{G}(1,10)$ with skewness 2 , and $\mathrm{G}(.25,40)$ with skewness 4 . The degrees of freedom for the chi-square distribution was chosen as $\chi^{2}(32)$ with skewness $.5, \chi^{2}(8)$ with skewness $1, \chi^{2}(2)$ with skewness 2 , and $\chi^{2}(.5)$ with skewness 4 . Similarly, the parameters for the log normal distribution were chosen as $\log$ normal $(2.25, .314)$ with skewness 1 and $\log$ normal $(1.96, .833)$ with skewness 4 . Steps ii and iv were repeated $\mathrm{M}$ times with $\mathrm{B}=2000$ and with sample sizes ranging from 5 to 50 . The confidence level for the simulation study is 0.95 . The number of simulation runs $(\mathrm{M})$ was determined following Kleijnen et al (1986). The number of replications needed to estimate the actual $\alpha$ error within $10 \%$ with $90 \%$ probability is 


$$
R=100(1.645)^{2}\left(\frac{1-\alpha}{\alpha}\right)=2435
$$

where $\alpha=0.1$. Therefore, in this study the number of replications $(M)$ was chosen as 2500.

\subsection{Results}

The results for this study have been presented in chart form (see Figures 1-6) in this section and in table form (see Tables A1-A12) in the Appendix. These tables give estimated confidence coefficients, average widths, and coefficient of variations using the gamma, chi square, and log normal distributions with various sample sizes (n) and skewness levels. Confidence coefficients versus sample sizes for gamma $(4,2.5)$ with skewness $=1$ and gamma $(0.25,40)$ with skewness $=4$ are presented in Figure 1 and 3 respectively. Widths versus sample sizes for $\operatorname{Gamma}(4,2.5)$ with skewness $=1$ and gamma $(0.25,40)$ with skewness $=4$ are presented in Figure 2 and 4 respectively. Figures 5 and 6 present confidence coefficients across skewness levels for fixed $n=10$ and $n=50$ using the gamma distribution; however, these two figures do not include the median bootstrap- $t$ and mad bootstrap- $t$ which we are only briefly discussing.

From the simulation study (both Figures 1 to 6 and Tables A1 to A12), we observed that the nominal coverage probability for the Student- $t$ drops below 0.95 for skewness levels as low as 1 . For small samples and for slightly to moderately skewed distributions, many confidence intervals outperform the Student- $t$ in terms of width. We 
also observe that the median- $t$ outperforms the Student $t$ in terms of coverage probability (see Figures 1,2 and 5). In terms of widths for slightly to moderately skewed distributions, six confidence intervals have smaller widths than the Student- $t$. These confidence intervals are the mad bootstrap- $t$, mad- $t$, mad $T_{1}, \mathrm{BCA}$, bootstrap- $t$, and median bootstrap- $t$ in order of increasing width (see Figure 2).

Additionally, we can see that the Student- $t$ and Johnson- $t$ perform about the same in terms of both coverage probability and width for slightly to moderately skewed distributions (see Appendix). Coverage probabilities for the median-t are slightly higher than the Student- $t$ and Johnson- $t$ but widths are also slightly wider for any sample size. For the mad- $t$, coverage probabilities are not as high as the previous three but widths are much smaller. The bootstrap methods have coverage probabilities which are much lower than the classical methods (except for the mad- $t$ ) however widths are smaller and comparable to the mad-t. The transformation methods outperform the bootstrap methods in terms of higher coverage probability but they do not do as well as the classical methods (except for the mad- $t$ ). The average widths for the transformation methods are wider than both the classical and bootstrap methods. Overall, the bootstrap methods perform the best in terms of having a smaller width, especially the mad bootstrap- $t$ which outperforms all other confidence intervals. In terms of coverage probability, the Student$t$, Johnson- $t$, and median- $t$ perform better than the rest.

For small samples from moderately to highly skewed distributions, the Student- $t$ is performing much worse than it is for lower skewness levels in terms of both coverage probability and width (see Figure 5 and Appendix). Many confidence intervals outperform the Student- $t$ in terms of having smaller average widths or having higher 
coverage probabilities for moderately to highly skewed distributions (see Figures 3, 4 and 5). In terms of coverage probability, the median $T_{3}$ has the highest coverage probability for small samples followed by median $T_{1}, T_{1}$, and $T_{3}$. In terms of widths, the following confidence intervals have smaller widths than the Student- $t$ : mad bootstrap-t, mad-t, bootstrap- $t$, BCA, mad $T_{3}$, median bootstrap- $t$, and mad $T_{1}$ in order of increasing width (see Figure 4).

We also observe that the Student- $t$ and Johnson- $t$ perform about the same in terms of both coverage probability and width for moderately to highly skewed distributions (see Appendix). Coverage probabilities for the median- $t$ are slightly higher than the Student- $t$ and Johnson- $t$ but widths are wider. The mad- $t$ has lower coverage probabilities than the previous three but widths are much smaller. The bootstrap methods have coverage probabilities which are slightly lower than the classical methods (except the mad- $t$ ). Among the bootstrap methods, only the mad bootstrap- $t$ has coverage probabilities which are lower then the mad- $t$. In terms of width, the bootstrap methods have smaller widths than the classical methods. The transformation methods outperform the bootstrap and classical methods in terms of higher coverage probability but their average widths are much wider than the other methods. Overall, the bootstrap methods perform the best in terms of smaller width, especially the mad bootstrap- $t$ which outperforms all other confidence intervals for all skewness levels. In terms of coverage probability, the transformation methods perform the best specifically the median $T_{3}$ and median $T_{1}$.

All the confidence intervals, except mad- $t$ (and modified mad confidence intervals), have coverage probabilities which converge to 0.95 as the sample size 
increases (see Figures 1 and 3). The mad- $t$ and modified mad confidence intervals have confidence coefficients which are relatively stable and less than the nominal level 0.95 as sample size increases. The simulation study indicates that the average width drops for all intervals as well as sample sizes as skewness increases (see Figures 2 and 4). Results for the small sample size and moderately to highly skewed distributions are of particular interest in this thesis. For small samples and moderately skewed distributions, median-t, Johnson- $t$ and Student- $t$ have the highest coverage probabilities and the mad bootstrap- $t$, BCA, bootstrap-t, and mad- $t$ have the smallest width. For small sample and highly skewed distributions, $T_{1}, T_{3}$, median $T_{1}$ and median $T_{3}$ have the highest coverage probabilities and the mad bootstrap- $t$, mad- $t$, BCA and mad $T_{3}$ have the smallest widths.

The best confidence intervals for moderately to highly skewed distributions will have the highest coverage probabilities with the least amount of error. However, the confidence intervals with high coverage probabilities have wider widths than the confidence intervals with low coverage probabilities. In terms of high coverage probability, the median $T_{3}$ performs the best followed by median $T_{1}, T_{1}, T_{3}$, median- $t$, Johnson- $t$, Student- $t$, median bootstrap- $t$, bootstrap- $t$, BCA, mad $T_{1}$, mad- $t$, $\operatorname{mad} T_{3}$, and mad bootstrap- $t$. In terms of having a smaller width, mad bootstrap-t, performs the best followed by mad- $t$, mad $T_{3}$, bootstrap- $t$, BCA, median bootstrap- $t$, mad $T_{1}$, Student- $t$, Johnson- $t$, median- $t, T_{3}$, median $T_{3}, T_{1}$ and median $T_{1}$.

The best median confidence interval in terms of coverage probability is the median $T_{3}$ because its coverage probabilities remain stable across skewness levels and 
sample sizes although its widths are very wide (see Figures 5-6 and Appendix).

Additionally, the modified mad confidence intervals outperform their respective confidence intervals in terms of having smaller widths however the sacrifice is a large drop in coverage probability. 


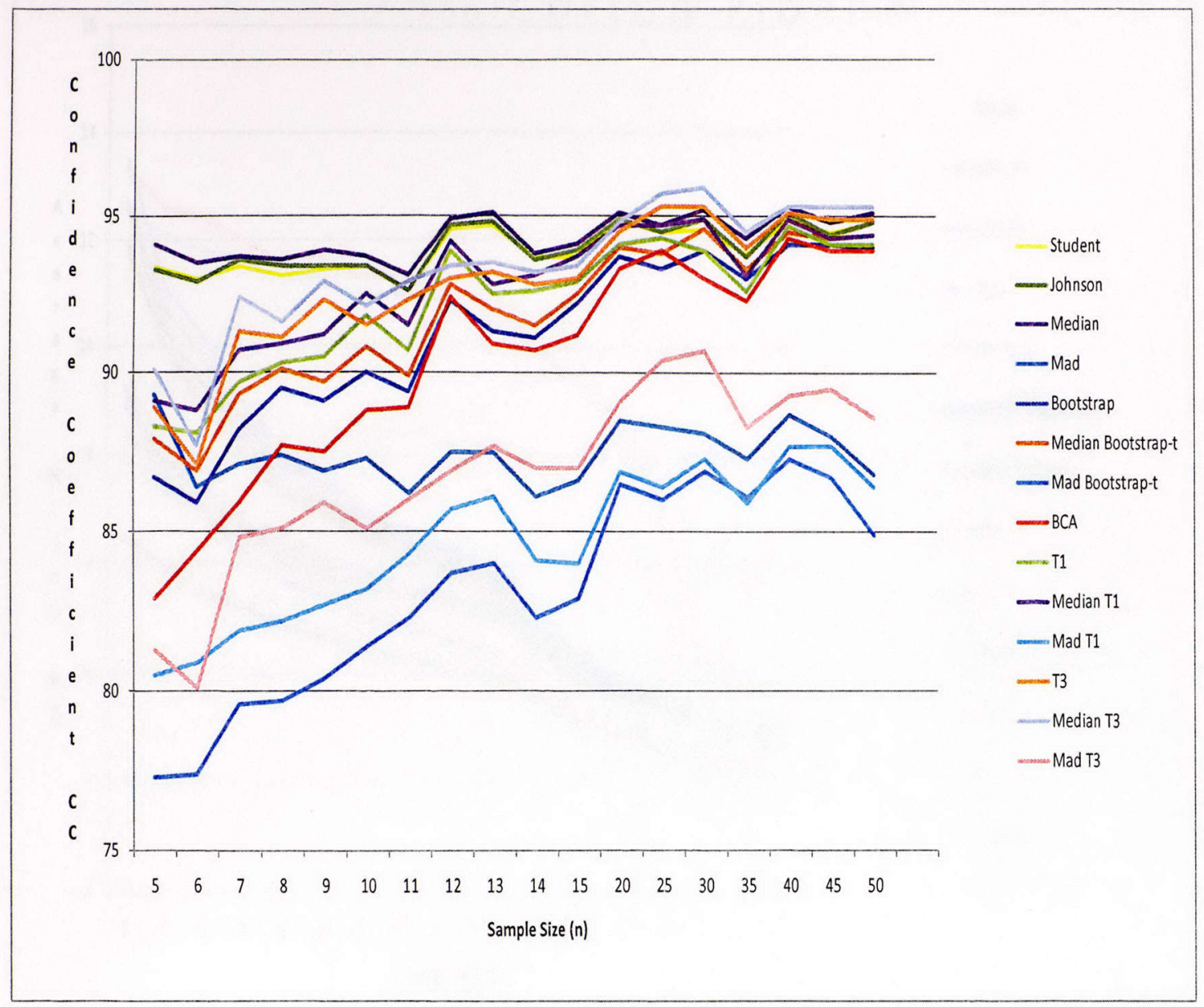

Figure 1: Confidence Coefficients for Gamma $(4,2.5)$ with Skewness $=1$ including Median and Mad Bootstrap- $t$ 


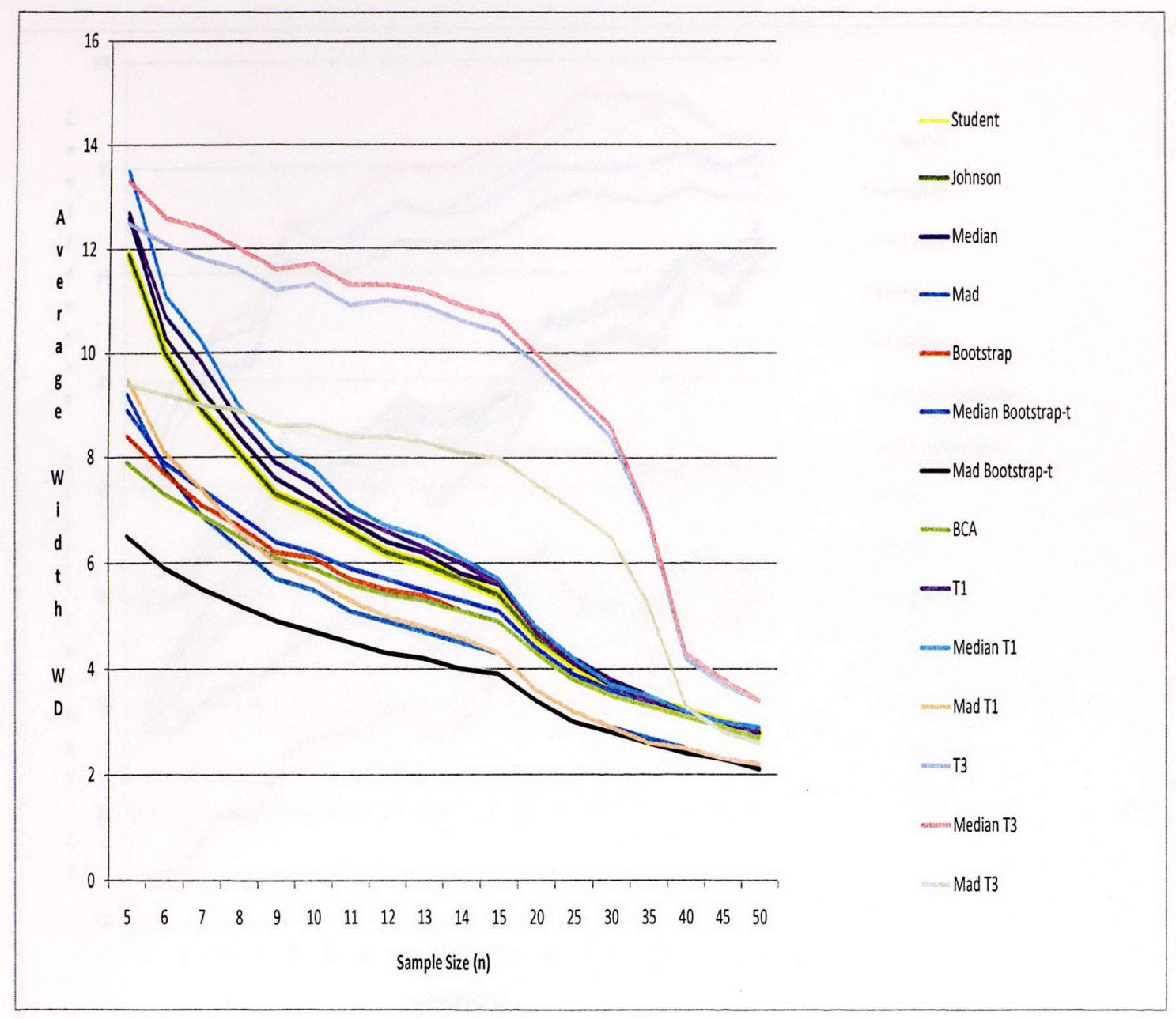

Figure 2: Average Widths for Gamma $(4,2.5)$ with Skewness $=1$ including Median and Mad Bootstrap- $t$ 


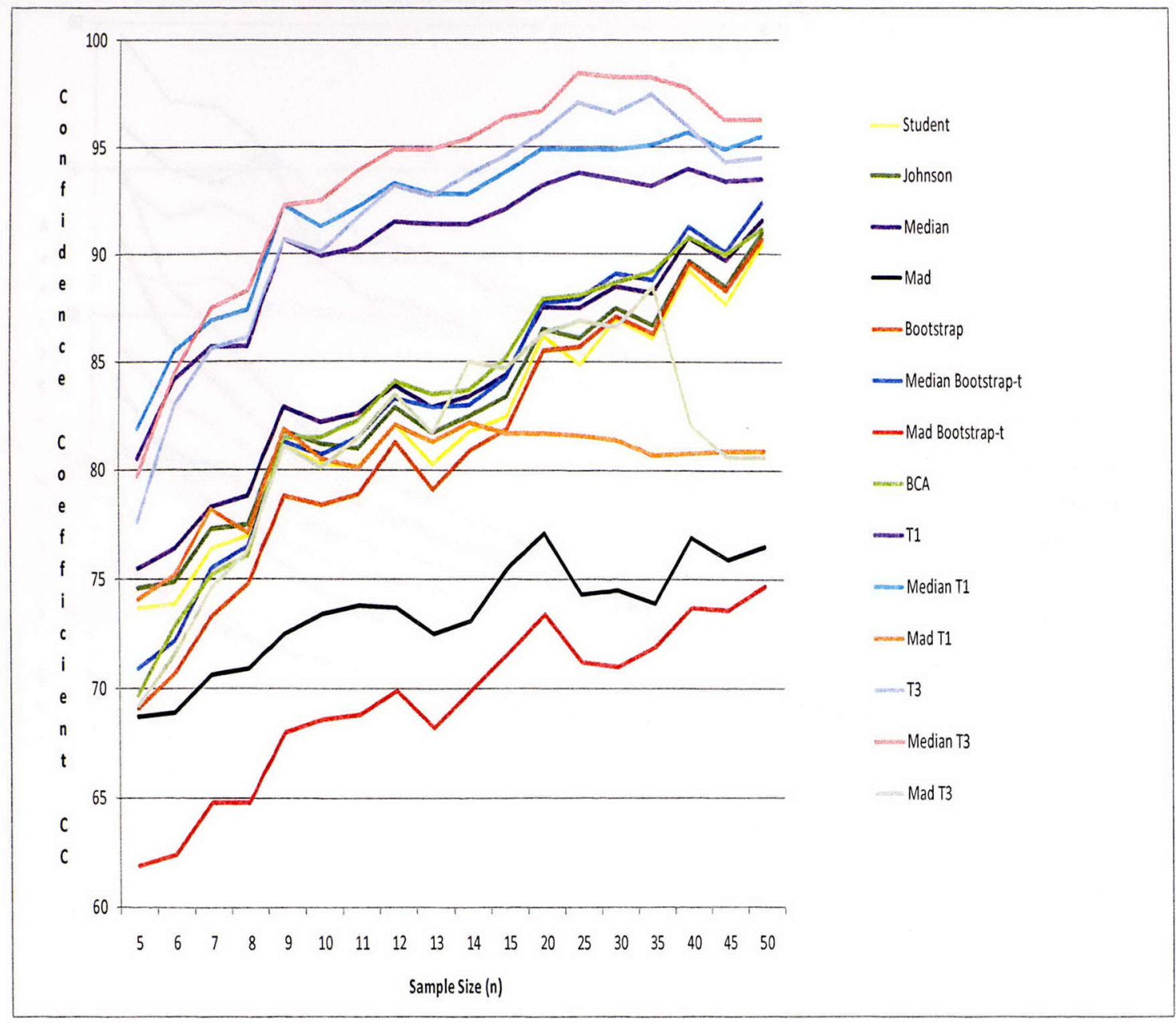

Figure 3: Confidence Coefficients for $\operatorname{Gamma}(.25,40)$ with Skewness $=4$ including Median and Mad Bootstrap- $t$ 


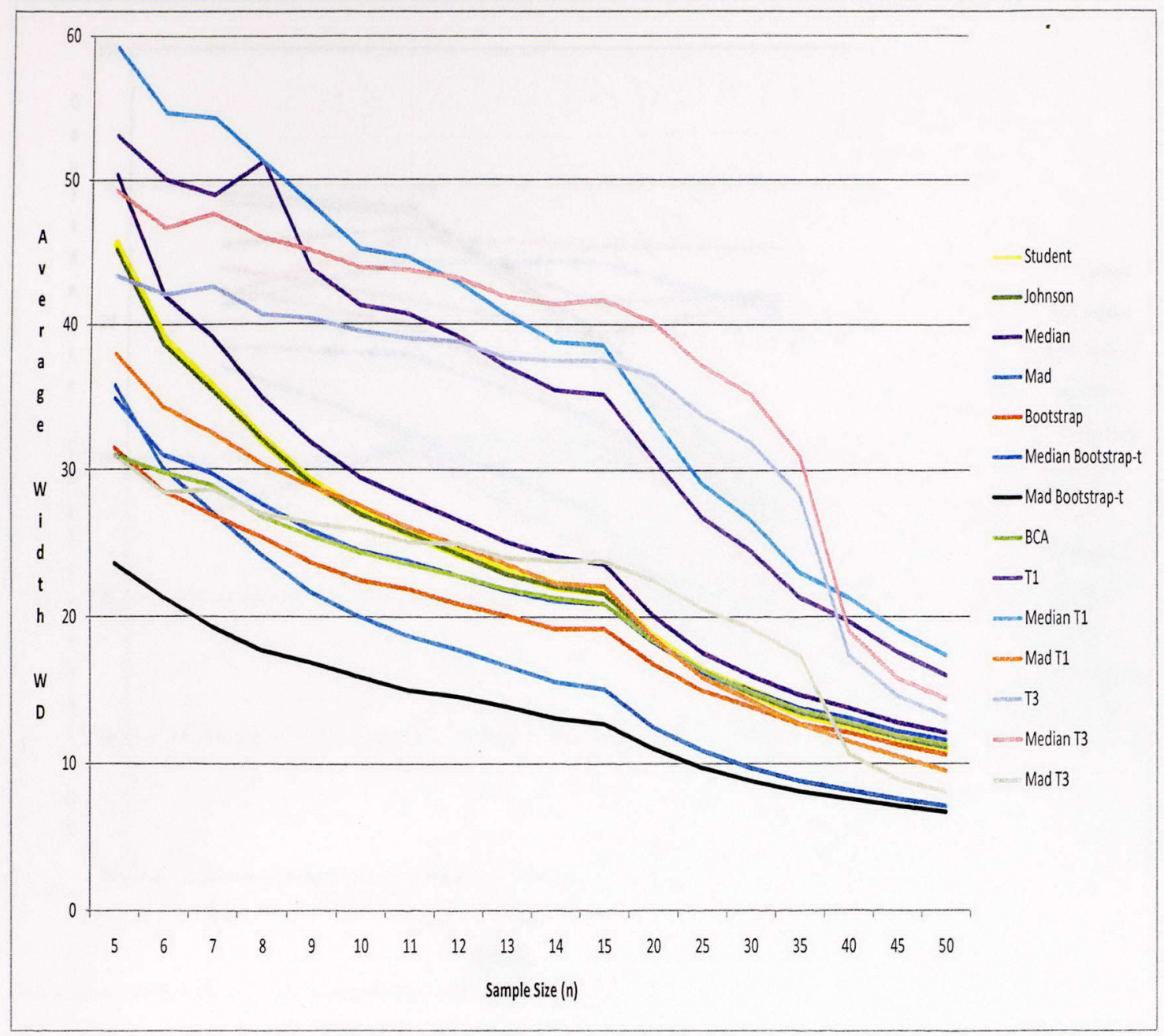

Figure 4: Average Widths for Gamma $(.25,40)$ with Skewness $=4$ including Median and Mad Bootstrap- $t$ 


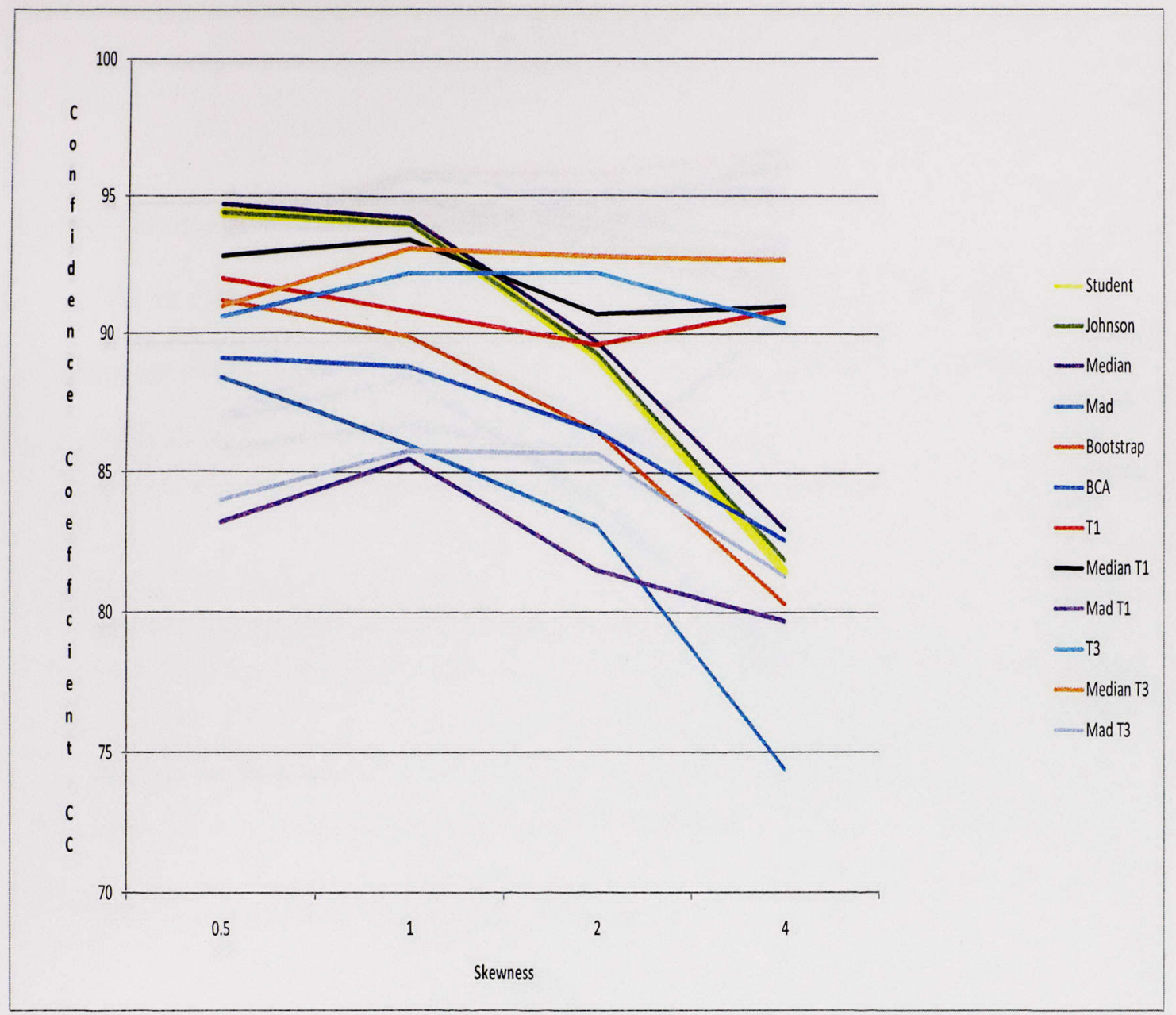

Figure 5: Confidence Coefficients for Gamma with Various Skewness levels and Fixed Sample Size $(n=10)$ 


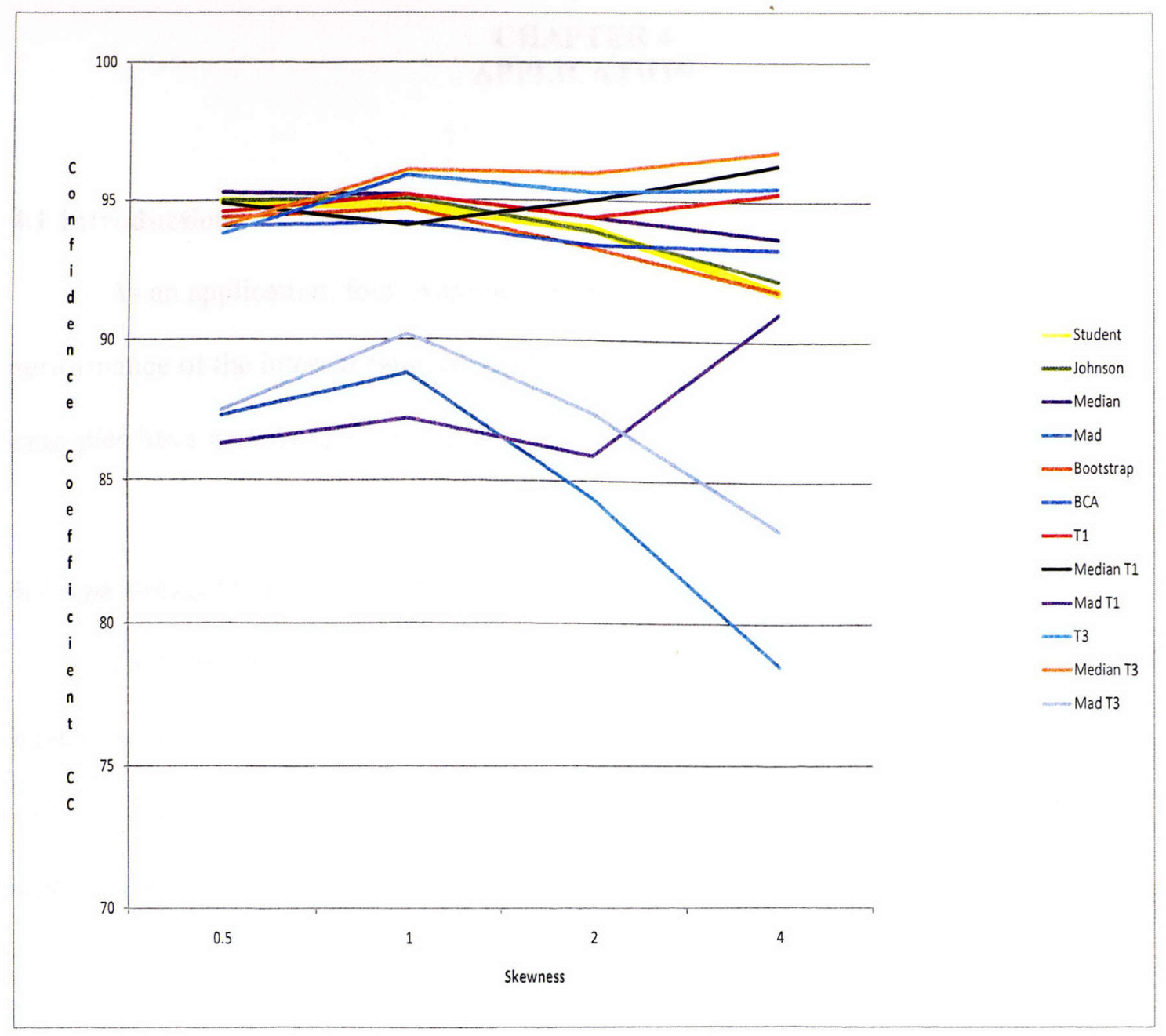

Figure 6: Confidence Coefficients for Gamma with Various Skewness levels and Fixed Sample Size $(n=50)$ 


\section{CHAPTER 4 \\ APPLICATION}

\subsection{Introduction}

As an application, four examples have been considered to illustrate the performance of the interval estimators which have been considered in Chapter 3. These examples have various sample sizes and levels of skewness.

\subsection{Age-Related Cataract Mortality}

Thirty seven different categories of contract lens wearers were analyzed for agerelated cataracts which is associated with accelerated aging (Williams et. al., 2002, pg. 129 , Table 1); the following data represent the number of premature deaths associated with age-related cataracts in each category:

$$
\begin{gathered}
24,54,60,67,82,99,108,111,126,146,166,212,247,262, \\
282,284,319,360,362,390,425,438,438,445,469,478 \\
480,501,517,520,560,767,769,1021,1109,1269,1281
\end{gathered}
$$

We want to find the average number of deaths associated with age-related cataracts among contact lens wearers. The sample is positively skewed with skewness $=1.27$, and mean $=412.11$. A histogram of the data in Figure 7 is showing positive skewness. The proposed confidence intervals and their widths have been given in Table 1. 


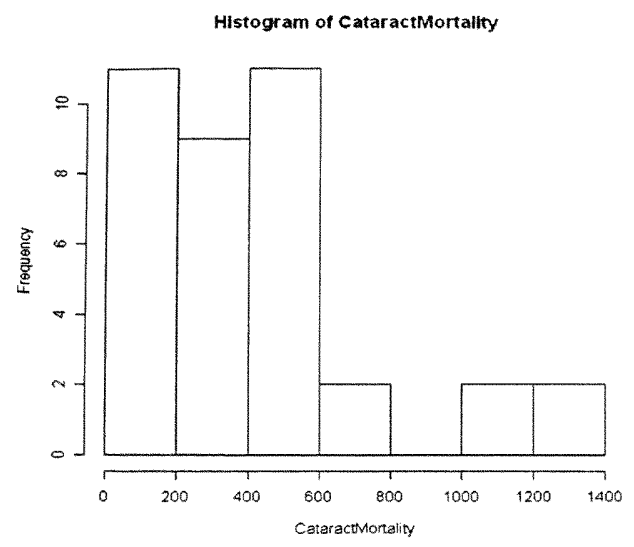

Figure 7: Histogram of Age-Related Cataract Mortality Data

Table 1: 95\% Confidence Intervals for Age-Related Cataract Mortality Data

\begin{tabular}{|c|c|c|}
\hline Method & Confidence Interval & Width \\
\hline Student- $t$ & $(301.96,522.26)$ & 220.30 \\
\hline Johnson- $t$ & $(303.85,524.14)$ & 220.30 \\
\hline Median- $t$ & $(300.66,523.55)$ & 222.89 \\
\hline Mad- $t$ & $(331.35,492.87)$ & 161.52 \\
\hline Bootstrap- $t$ & $(311.73,519.16)$ & 207.44 \\
\hline Median Bootstrap- $t$ & $(310.53,520.41)$ & 209.87 \\
\hline Mad Bootstrap- $t$ & $(338.91,491.01)$ & 152.09 \\
\hline BCA Bootstrap & $(317.47,532.51)$ & 215.04 \\
\hline$T_{1}$ Transformation & $(319.07,540.33)$ & 221.27 \\
\hline Median $T_{1}$ Transformation & $(317.97,514.84)$ & 162.23 \\
\hline Mad $T_{1}$ Transformation & $(343.89,506.84)$ & 329.08 \\
\hline$T_{3}$ Transformation & $(329.77,658.85)$ & 332.95 \\
\hline Median $T_{3}$ Transformation & $(328.80,661.75)$ & 241.28 \\
\hline Mad $T_{3}$ Transformation & $(351.74,593.02)$ & \\
\hline
\end{tabular}


From Table 1, we observe that the mad bootstrap- $t$ has the smallest width followed by mad- $t$ and the median $T_{3}$ transformation has the highest width. Both the proposed median bootstrap- $t$ and mad bootstrap- $t$ have shorter widths compared to the corresponding median- $t$ and mad- $t$ respectively. Student- $t$, Johnson- $t$, median- $t, T_{1}$ transformation and median $T_{1}$ transformation have approximately equal amounts of width and are relatively similar. The example supported the simulation study to some extent.

\subsection{Psychotropic Drug Exposure}

To study the average use of psychotropic drugs from non-antipsychotic drug users, the number of users of psychotropic drugs was reported for twenty different categories of drugs (Johnson and McFarland, 1993, pg. 438, Table 3); the following data represent the number of users:

$$
\begin{gathered}
43.4,24,1.8,0,0.1,170.1,0.4,150.0,31.5,5.2 \\
35.7,27.3,5,64.3,70,94,61.9,9.1,38.8,14.8
\end{gathered}
$$

We want to find the average number of users of psychotropic drugs for non-antipsychotic drug users.

The sample is positively skewed with skewness $=1.57$, and mean $=42.37$ A histogram of the data values showing its positive skewness is given in Figure 8. The proposed confidence intervals and their widths have been given in Table 2 . 


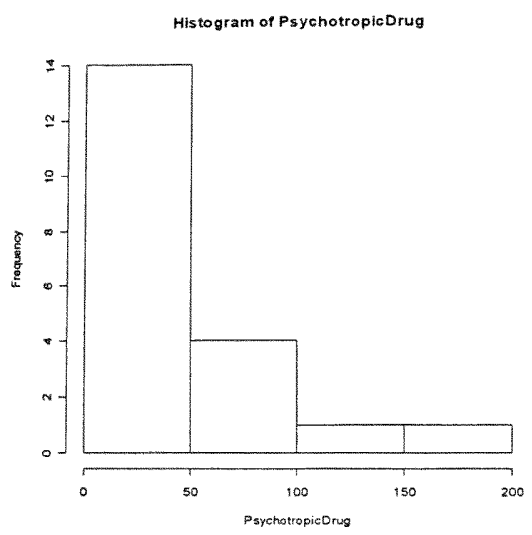

Figure 8: Histogram of Psychotropic Drug Exposure Data

Table 2: 95\% Confidence Intervals for Psychotropic Drug Exposure Data

\begin{tabular}{|c|c|c|}
\hline Method & Confidence Interval & Width \\
\hline Student- $t$ & $(19.70,65.04)$ & 45.33 \\
\hline Johnson- $t$ & $(20.34,65.67)$ & 45.33 \\
\hline Median- $t$ & $(18.86,65.88)$ & 47.01 \\
\hline Mad- $t$ & $(25.66,59.08)$ & 33.43 \\
\hline Bootstrap- $t$ & $(22.53,65.25)$ & 42.72 \\
\hline Median Bootstrap- $t$ & $(21.82,66.12)$ & 44.30 \\
\hline Mad Bootstrap- $t$ & $(27.58,59.08)$ & 31.50 \\
\hline BCA Bootstrap & $(26.67,69.42)$ & 42.75 \\
\hline$T_{1}$ Transformation & $(25.16,73.51)$ & 48.35 \\
\hline Median $T_{1}$ Transformation & $(24.53,74.67)$ & 50.14 \\
\hline Mad $T_{1}$ Transformation & $(29.68,65.33)$ & 35.65 \\
\hline$T_{3}$ Transformation & $(27.10,125.06)$ & 97.96 \\
\hline Median $T_{3}$ Transformation & $(26.53,128.12)$ & 101.59 \\
\hline Mad $T_{3}$ Transformation & $(31.11,103.34)$ & 72.23 \\
\hline
\end{tabular}


From Table 2, we observe that the mad bootstrap- $t$ again has the smallest width followed by mad- $t$ and the median $T_{3}$ transformation again has the widest width. Both the proposed median bootstrap- $t$ and mad bootstrap- $t$ have shorter widths compared to the corresponding median- $t$ and mad- $t$ respectively. Most of the confidence intervals have short widths with the exception of the $T_{3}$ transformation, median $T_{3}$ transformation, and the mad $T_{3}$ transformation which have the widest widths. The example supported the simulation study to some extent.

\subsection{Mosquito Survival Rates}

To study Mosquito survival rates in a wet climate, eight survival times were reported (Charlwood et. al., 1985, pg. 1011, Table 3); the following data represent the time of death:

$$
0.539,0.292,0.090,0.044,0.010,0.010,0.010,0.031
$$

We want to find the average survival time.

The sample is positively skewed with skewness $=1.83$, and mean $=0.13$. A histogram of the data values showing its positive skewness is given in Figure 9. The proposed confidence intervals and their widths have been given in Table 3 . 


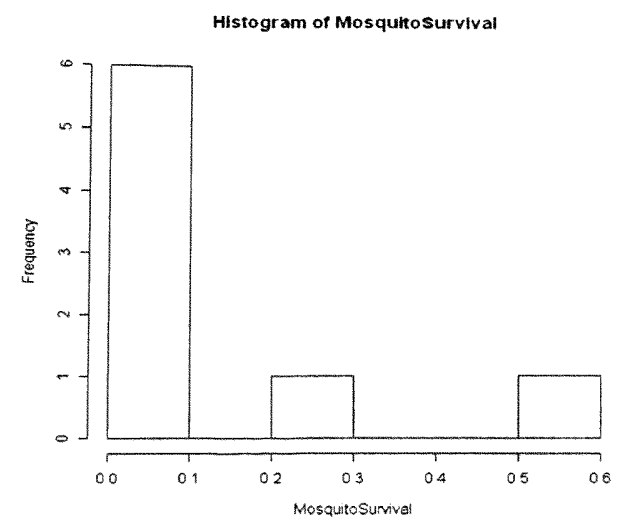

Figure 9: Histogram of Mosquito Survival Rates Data

Table 3: 95\% Confidence Intervals for Mosquito Survival Rates Data

\begin{tabular}{|c|c|c|}
\hline Method & Confidence Interval & Width \\
\hline Student- $t$ & $(-0.0315,0.2880)$ & 0.3194 \\
\hline Johnson- $t$ & $(-0.0242,0.2953)$ & 0.3194 \\
\hline Median- $t$ & $(-0.0509,0.3074)$ & 0.3583 \\
\hline Mad- $t$ & $(0.0082,0.2483)$ & 0.2401 \\
\hline Bootstrap- $t$ & $(0.0176,0.2721)$ & 0.2546 \\
\hline Median Bootstrap- $t$ & $(0.0041,0.2895)$ & 0.2855 \\
\hline Mad Bootstrap- $t$ & $(0.0452,0.2366)$ & 0.1914 \\
\hline BCA Bootstrap & $(0.0369,0.3254)$ & 0.2885 \\
\hline$T_{1}$ Transformation & $(0.0343,0.6590)$ & 0.6248 \\
\hline Median $T_{1}$ Transformation & $(0.0228,0.7235)$ & 0.7007 \\
\hline Mad $T_{1}$ Transformation & $(0.0576,0.5273)$ & 0.4697 \\
\hline$T_{3}$ Transformation & $(0.0449,0.5220)$ & 0.4771 \\
\hline Median $T_{3}$ Transformation & $(0.0347,0.5698)$ & 0.5350 \\
\hline Mad $T_{3}$ Transformation & $(0.0656,0.4242)$ & 0.3587 \\
\hline
\end{tabular}


From Table 3, we observe that the mad bootstrap- $t$ has the smallest width

followed by mad- $t$, however this time the median $T_{1}$ transformation has the highest width. Again, both the proposed median bootstrap- $t$ and mad bootstrap- $t$ have shorter widths compared to the corresponding median- $t$ and mad- $t$ respectively. The widest widths are the median $T_{1}$ transformation, $T_{1}$ transformation, and median $T_{3}$ transformation. The example supported the simulation study to some extent.

\subsection{HIV-1 Prevalence}

The percentages of adults living with HIV-1 for fifteen regions of the world were reported (Hemelaar et. al., 2006, pg. W16, Table 1); the following data represent the HIV-1 prevalence rate for each region:

$$
0.6,2.3,0.6,0.3,0.7,0.9,0.3,0.1,0.2,0.3,4.5,5.7,4.4,4.8,17
$$

We want to find the average percentage of disorders for a region.

The sample is positively skewed with skewness $=2.67$, and mean $=2.8 \mathrm{~A}$ histogram of the data values showing its positive skewness is given in Figure 10. The proposed confidence intervals and their widths have been given in Table 4. 


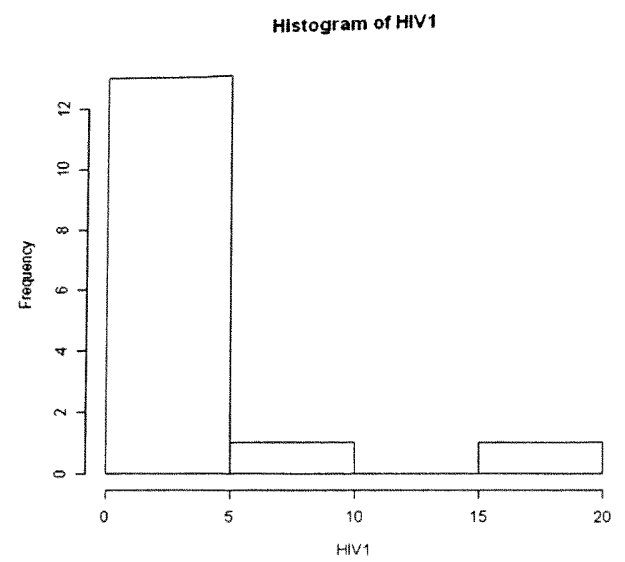

Figure 10: Histogram of HIV-1 Prevalence Data

Table 4: 95\% Confidence Intervals for HIV-1 Prevalence Data

\begin{tabular}{|c|c|c|}
\hline Method & Confidence Interval & Width \\
\hline Student- $t$ & $(0.41,5.28)$ & 4.87 \\
\hline Johnson- $t$ & $(0.54,5.41)$ & 4.87 \\
\hline Median- $t$ & $(0.12,5.57)$ & 5.45 \\
\hline Mad- $t$ & $(1.21,4.48)$ & 3.27 \\
\hline Bootstrap- $t$ & $(1.04,5.35)$ & 4.30 \\
\hline Median Bootstrap- $t$ & $(0.83,5.65)$ & 4.82 \\
\hline Mad Bootstrap- $t$ & $(1.62,4.52)$ & 2.90 \\
\hline BCA Bootstrap & $(1.41,6.50)$ & 5.10 \\
\hline$T_{1}$ Transformation & $(1.29,11.51)$ & 10.22 \\
\hline Median $T_{1}$ Transformation & $(1.11,12.56)$ & 6.88 \\
\hline Mad $T_{1}$ Transformation & $(1.80,8.68)$ & 9.63 \\
\hline$T_{3}$ Transformation & $(1.33,10.96)$ & 10.79 \\
\hline Median $T_{3}$ Transformation & $(1.15,11.94)$ & 6.48 \\
\hline Mad $T_{3}$ Transformation & $(1.83,8.30)$ & \\
\hline
\end{tabular}


From Table 4, we observe that the mad bootstrap- $t$ has the smallest width followed by mad- $t$ and the median $T_{1}$ transformation has the highest width. Both the proposed median bootstrap- $t$ and mad bootstrap- $t$ have shorter widths compared to the corresponding median- $t$ and mad- $t$ respectively. Student- $t$, Johnson- $t$, bootstrap- $t$ and median bootstrap- $t$ have short and approximately equal widths. The example supported the simulation study to some extent. 


\section{CHAPTER 5}

\section{CONCLUDING REMARKS}

This thesis reviews and proposes several confidence intervals for the mean of a positively skewed distribution using the classical, bootstrap and transformation approaches. A simulation study has been conducted to compare the performance of the

interval estimators: Student- $t$, Johnson- $t$, median- $t$, mad- $t$, bootstrap- $t$, BCA, $T_{1}, T_{3}$, and six new transformations which are the median bootstrap-t, median $T_{1}$, median $T_{3}$, mad bootstrap-t, mad $T_{1}$, and mad $T_{3}$. A good confidence interval will have high coverage probability and a small width. However, it is hard to find a confidence interval which satisfies both of these characteristics at the same time. There is an inverse relationship between high coverage probability and low width. Therefore, the experimenters or practitioners should decide whether coverage probability or width is most important to their study then choose a confidence interval which sacrifices very little of the opposing factor.

The simulation study shows that the best confidence interval based on coverage probability for moderately to highly skewed data is the median $T_{3}$ followed by median $T_{1}, T_{1}, T_{3}$, median- $t$, Johnson- $t$, Student- $t$, median bootstrap- $t$, bootstrap- $t$, BCA, mad $T_{1}$, mad- $t$, mad $T_{3}$, and mad bootstrap- $t$. The best confidence interval for moderately to highly skewed data based on width is the mad bootstrap-t, followed by mad- $t$, mad $T_{3}$, bootstrap- $t$, BCA, median bootstrap- $t$, mad $T_{1}$, Student- $t$, Johnson- $t$, median- $t, T_{3}$, 
median $T_{3}, T_{1}$ and median $T_{1}$. Four real life examples are analyzed which supported these results to some extent.

The proposed confidence intervals performed well in the sense that they improved their respective confidence intervals in terms of either coverage probability or width. Specifically, the modified median confidence intervals improved their confidence intervals in terms of coverage probability and the modified mad confidence intervals improved their intervals in terms of width. This improvement applied to all of the modified confidence intervals and gives some evidence that the median and mad confidence intervals can improve other existing intervals. Furthermore, these modifications are very easy to implement compared to other existing intervals.

Even though we have classified the intervals based on the higher coverage probability or shorter width, overall, the following intervals performed well in the sense of both coverage probability and shorter widths: the median- $t$, Johnson- $t$, Student- $t$, median bootstrap- $t$, bootstrap- $t$, and the BCA. Finally, the proposed interval estimation methods performed well compared to some existing estimators. We also believe that the comparison among various confidence intervals helps us to find some good and useful interval estimators. It is also evident from the simulation study that the sample size 30 for the normal or $t$ interval is inadequate for highly skewed data. 


\section{REFERENCES}

Box, G. E. P. and Cox, D. R. (1964). An analysis of transformations, Journal of the Royal Statistical Society Series B, 26, 211-252.

Charlwood, J. D., Birley, M. H., Dagoro, H., Paru, R., and Holmes, P. R. (1985). Assessing survival rates of anopheles farauti (diptera: culicidae) from Papua New Guinea, Journal of Animal Ecology, 54, 1003-1016.

Cornish, E. A. and Fisher, R. A. (1937). Moments and cumulants in the specifications of Distributions, Revue of the International Statistics Instituters, 5, 307-327.

Efron, B. (1979). Bootstrap methods: another look at the Jackknife, Annals of Statistics, 7, 1-26.

Efron, B. (1982). The Jackknife, the Bootstrap, and Other Resampling Plans, SIAM, Philadelphia

Efron, B. (1987). Better Bootstrap confidence intervals, Journal of American Statistical Association, 82, 171-185.

Efron, B. and Tishirani, R. J. (1993). An Introduction to Bootstrap, Chapman and Hall, London, 162.

Hall, P. (1992). On the removal of skewness by transformation, Journal of the Royal Statistical Society, Series B 54, 221-228.

Hemelaar, J., Gouws, E., Ghys, P. D., and Osmanov, S. (2006). Global and regional distribution of HIV-1 genetic subtypes and recombinants in 2004, AIDS, 20, W13W23.

Johnson, N. J. (1978). Modified $t$ tests and confidence intervals for asymmetrical population, Journal of the American Statistical Association, 73, 536-544.

Johnson, R. E., and McFarland, B. H. (1993). Antipsychotic drug exposure in health maintenance organization, Medical Care, 31(5), 432-444.

Kleijnen, J. P. C., Kloppenburg, G. L. J., and Meeuwsen, F. L. (1986). Testing the mean of an asymmetric population: Johnson's modified $t$ test revisited, Communications in Statistics - Simulation and Computation, 15(3), 715-732.

Oakes, M. (1990). Statistical Inference, Epidemiological Resources, Newton Lower Falls, MA. 
Rothman K. J. and Greenland S. (1998). Modern Epidemiology, Lippincott-Raven, Philadelphia, 2, 183-199.

Shao J. and Tu D. (1995). The Jackknife and Bootstrap, Springer, New York, 135-140.

Shi, W. and Kibria, B. M. G. (2007). On some confidence intervals for estimating the mean of a skewed population, International Journal of Mathematical Education in Science and Technology, 38(3), 412-421.

Student. (1908). The probable error of a mean, Biometrika 6, 1-25.

Taylor, J. M. G. (1986). The retransformed mean after a fitted power transformation, Journal of the American Statistical Association, 81(393), 114-118.

Visintainer, P. F. and Tejani, N. (1998). Understanding and using confidence intervals in clinical research, The Journal of Maternal-Fetal Medicine, 7, 201-206.

Wang, F. K. (2001). Confidence interval for the mean of non-normal data, Quality and Reliability Engineering International, 17, 257-267.

Williams, S. L., Ferrigno, L., Mora, P., Rosmini, F., and Maraini, G. (2002). Baseline cataract type and 10-year mortality in the Italian-American case-control study of age-related cataract, American Journal of Epidemiology, 156(2), 127-131.

Zhou, X. and Dinh, P. (2005). Nonparametric confidence intervals for the one- and twosample problems, Biostatistics, 6(2), 187-200. 
Table A1: Estimated Coverage Probabilities using Gamma(16, .625) with Skewness $=.5$ $(\mathrm{CC}=$ Confidence Coefficient, $\mathrm{WD}=$ Average Width, $\mathrm{CV}=$ Coefficient of Variation $)$

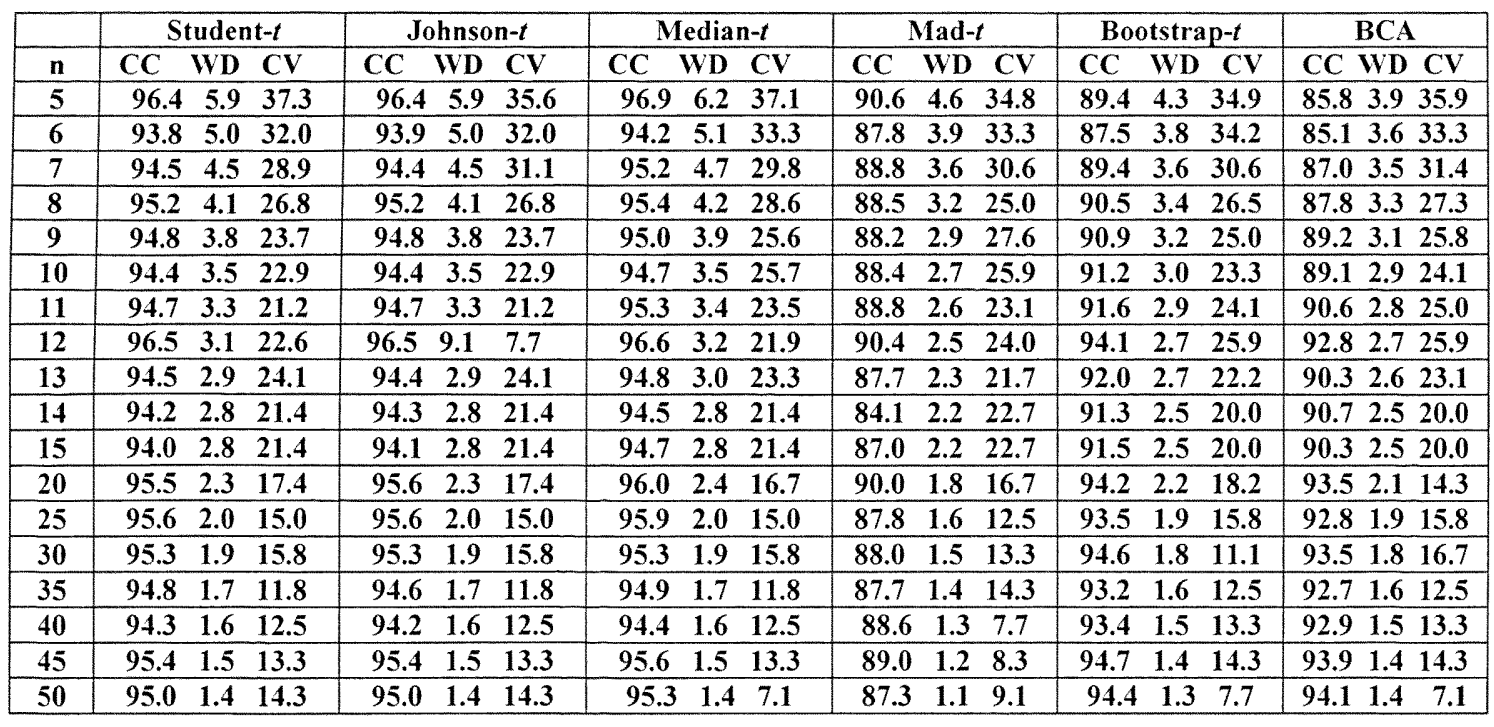

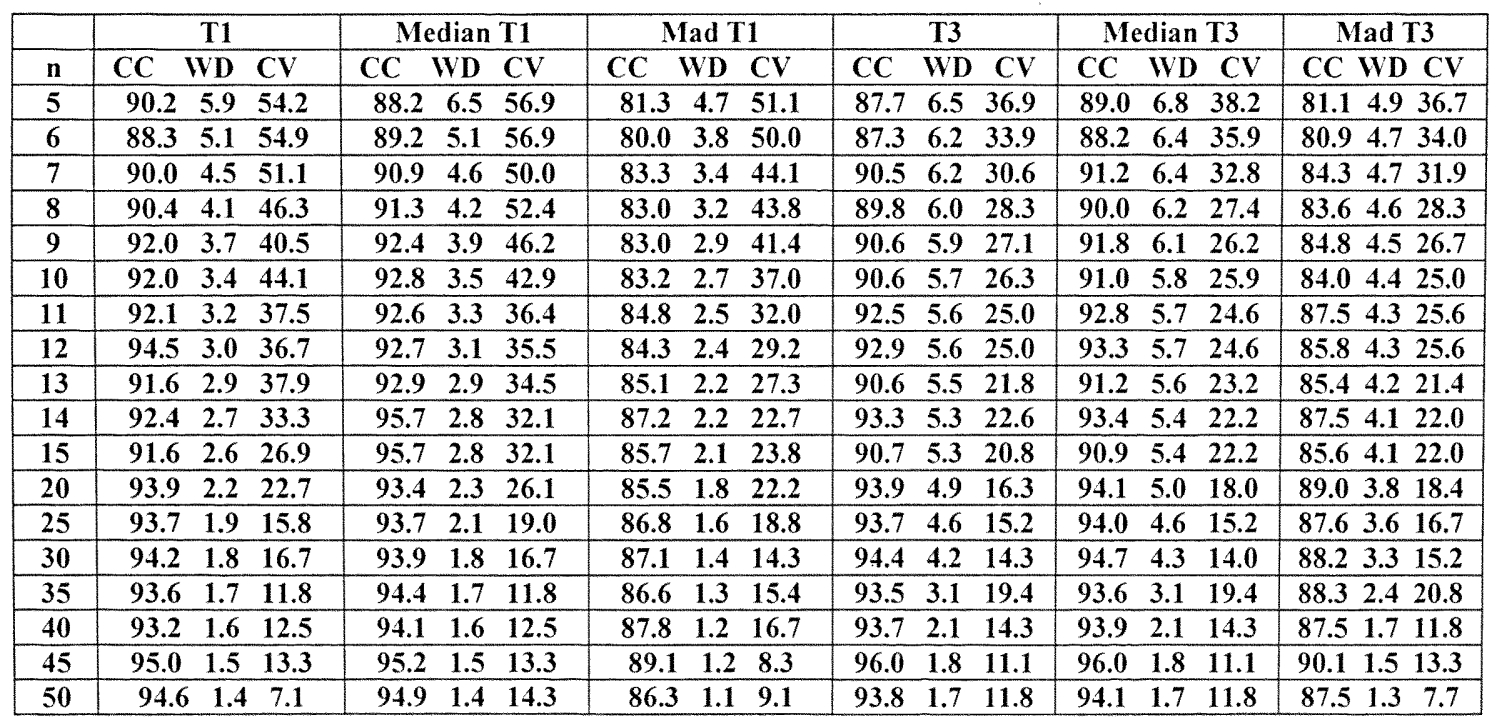


Table A2: Estimated Coverage Probabilities using $\operatorname{Gamma}(4,2.5)$ with Skewness $=1$ $(\mathrm{CC}=$ Confidence Coefficient, $\mathrm{WD}=$ Average Width, $\mathrm{CV}=$ Coefficient of Variation $)$

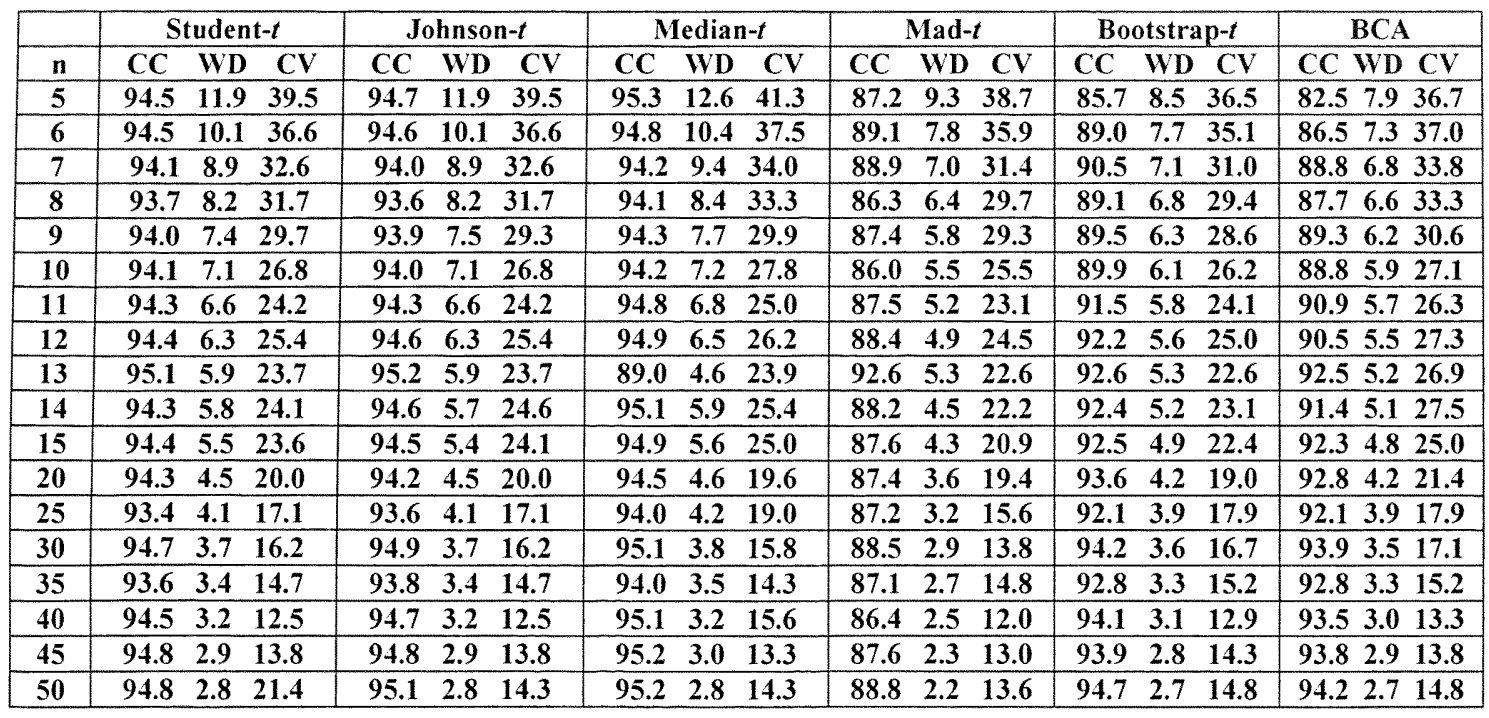

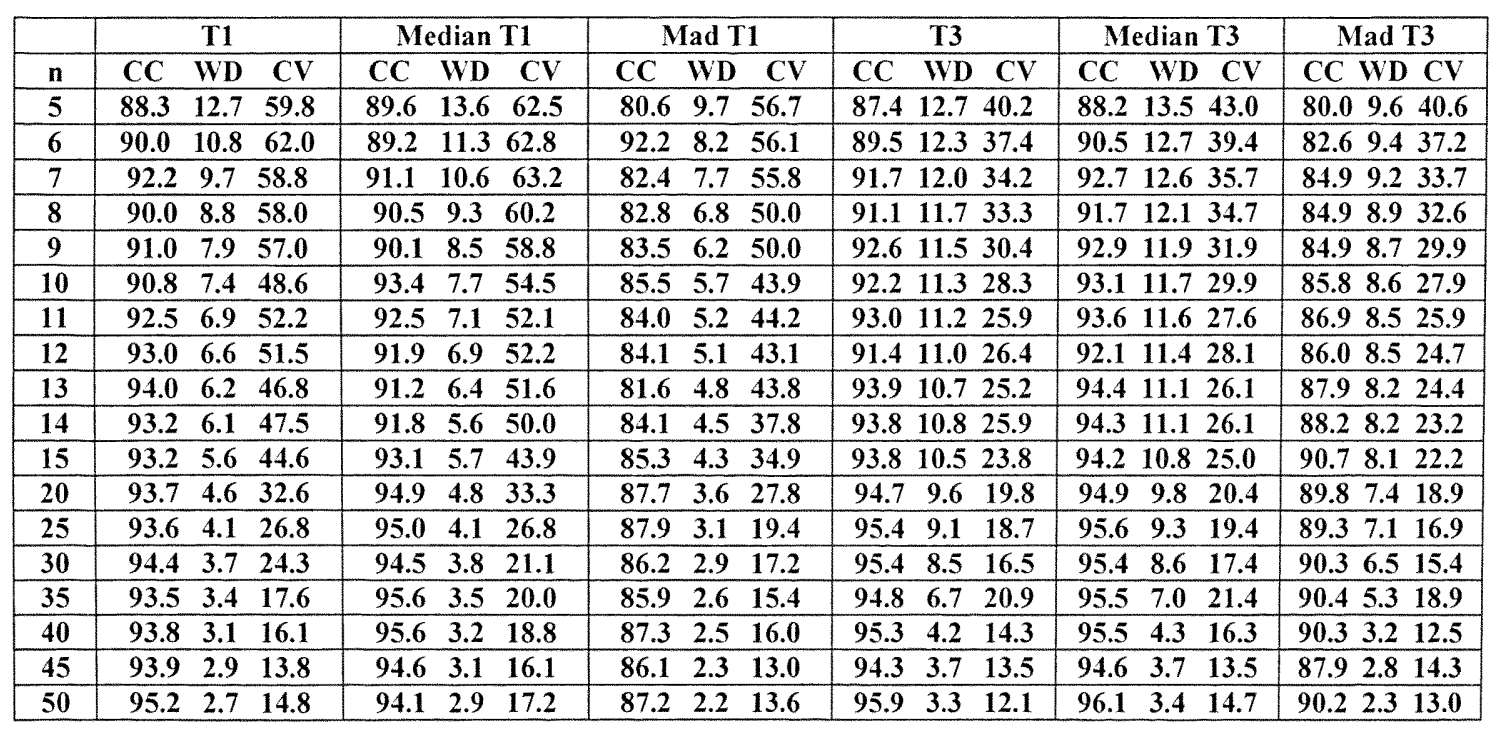


Table A3: Estimated Coverage Probabilities using Gamma(1,10) with Skewness $=2$ ( $\mathrm{CC}=$ Confidence Coefficient, $\mathrm{WD}=$ Average Width, $\mathrm{CV}=$ Coefficient of Variation $)$

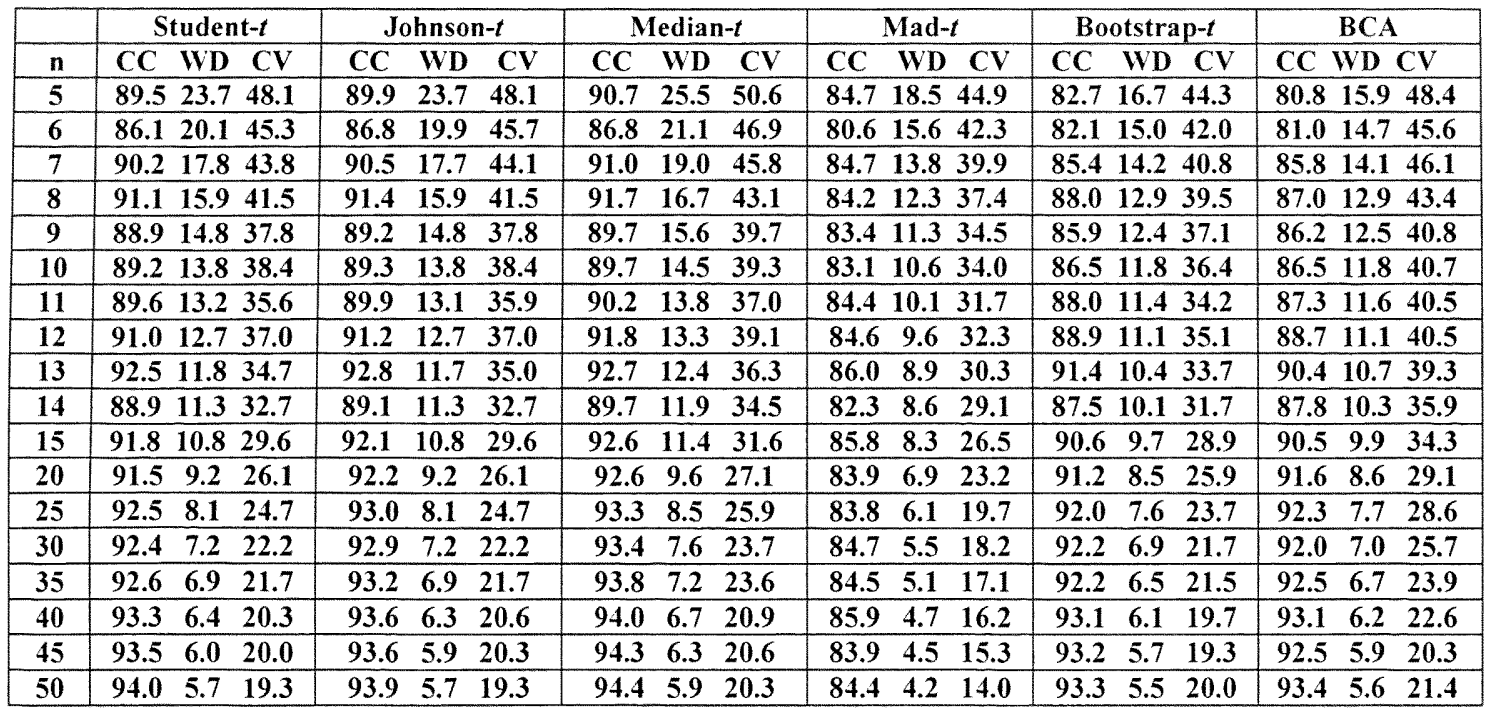

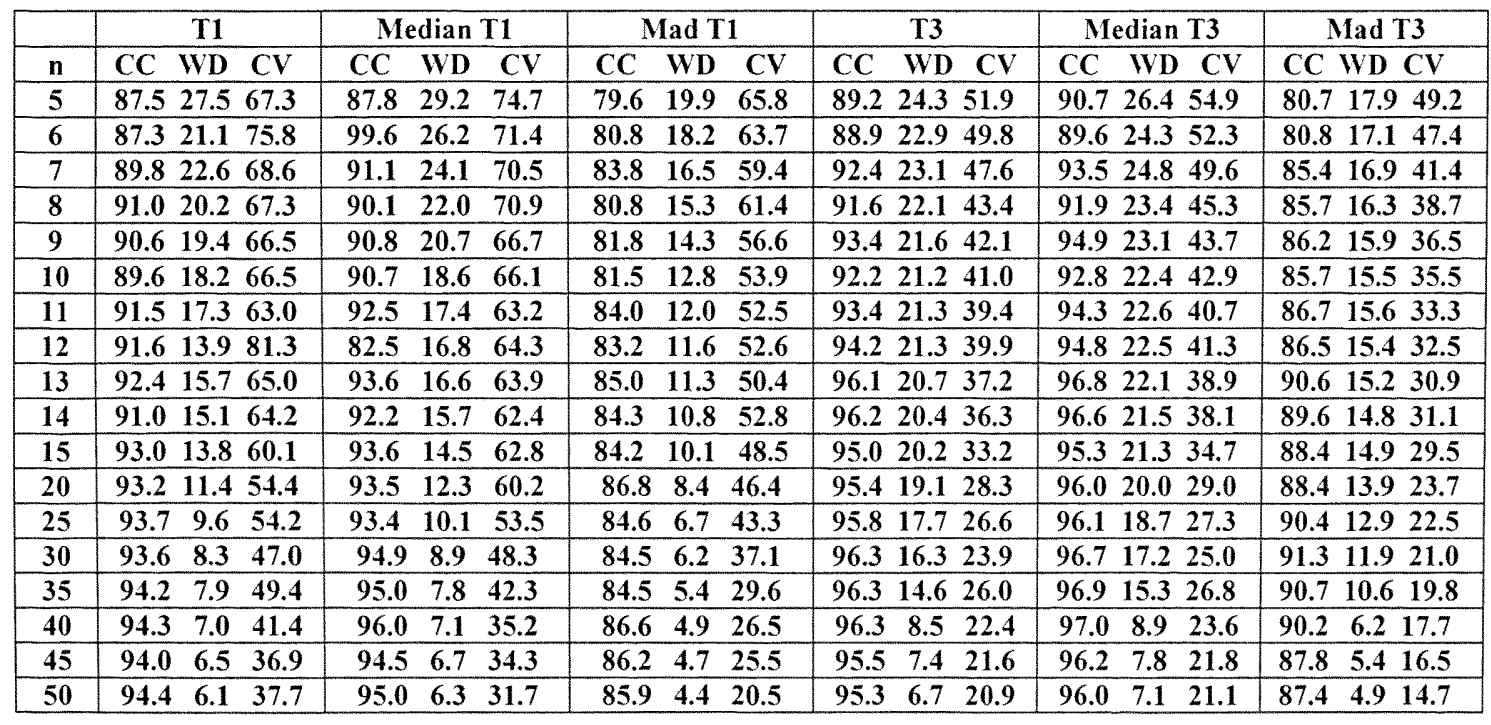


Table A4: Estimated Coverage Probabilities using $\operatorname{Gamma}(.25,40)$ with Skewness $=4$ ( $\mathrm{CC}=$ Confidence Coefficient, $\mathrm{WD}=$ Average Width, $\mathrm{CV}=$ Coefficient of Variation $)$

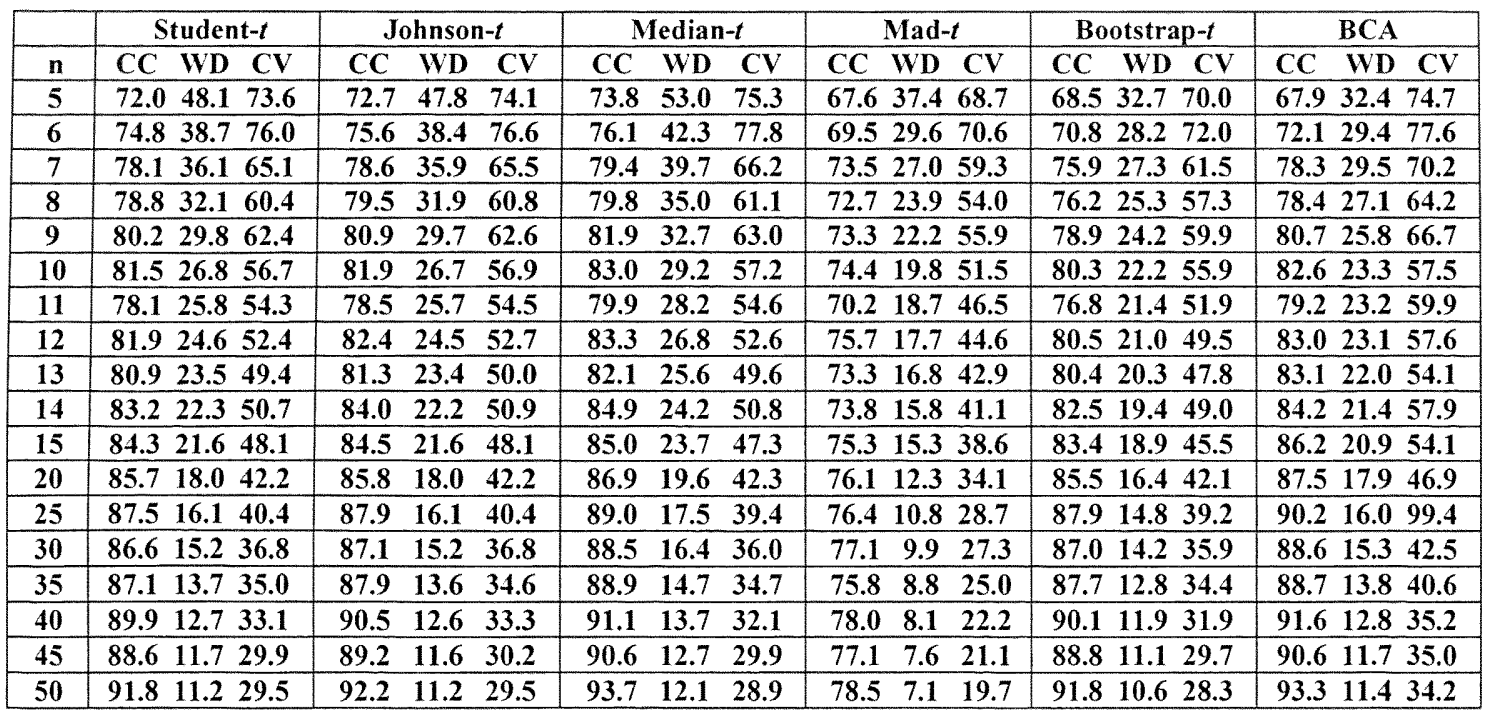

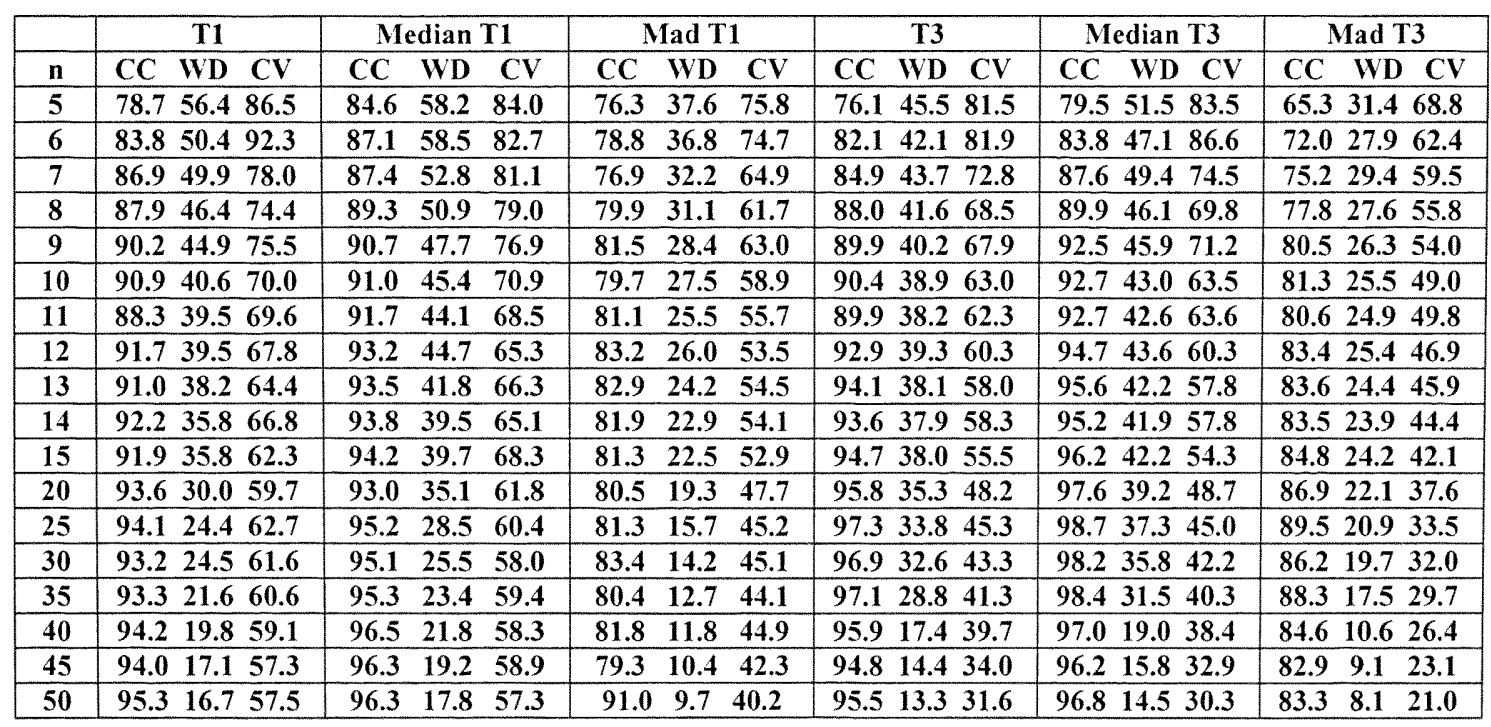


Table A5: Estimated Coverage Probabilities using $\chi^{2}(32)$ with Skewness $=.5(\mathrm{CC}=$ Confidence Coefficient, $\mathrm{WD}=$ Average Width, $\mathrm{CV}=$ Coefficient of Variation)

\begin{tabular}{|c|c|c|c|c|c|c|}
\hline & Student- $t$ & Johnson-t & Median- $t$ & Mad- $t$ & Bootstrap- $t$ & BCA \\
\hline $\mathbf{n}$ & $\mathrm{CC}$ WD $\mathrm{CV}$ & CC WD CV & $\mathrm{CC}$ WD $\mathrm{CV}$ & $\mathrm{CC}$ WD $\mathrm{CV}$ & CC WD CV & $\mathrm{CC}$ WD $\mathrm{CV}$ \\
\hline 5 & \begin{tabular}{|llll}
93.7 & 18.6 & 36.5 \\
\end{tabular} & $\begin{array}{llll}93.7 & 18.6 & 36.4 \\
\end{array}$ & 19.636 .9 & $\begin{array}{llll}86.9 & 14.4 & 5.8 \\
\end{array}$ & $84.7 \quad 13.3 \quad 35.0$ & $\begin{array}{lllll}82.2 & 12.4 & 35.4 \\
\end{array}$ \\
\hline 6 & $\begin{array}{llll}.5 & 16.1 & 31.7 \\
\end{array}$ & $\begin{array}{llll}94.3 & 16.1 & 31.6 \\
\end{array}$ & $\begin{array}{llll}94.7 & 16.6 & 32.2 \\
\end{array}$ & $\begin{array}{lllll}88.6 & 12.6 & 31.7\end{array}$ & $\begin{array}{llll}87.9 & 12.3 & 30.9\end{array}$ & $\begin{array}{llll}86.1 & 11.7 & 3\end{array}$ \\
\hline 7 & $14.5 \quad 29.7$ & \begin{tabular}{|lll}
5.1 & 14.5 & 29.7 \\
\end{tabular} & $\begin{array}{llll}95.6 & 15.1 & 30.4 \\
\end{array}$ & \begin{tabular}{|llll}
89.8 & 11.3 & 30.4 \\
\end{tabular} & $90.9 \quad 11.6 \quad 29.5$ & $88.8 \quad 11.13$ \\
\hline 8 & 13.126 .6 & $\begin{array}{ll}94.9 & 13.1 \\
\end{array}$ & $\begin{array}{llll}95.0 & 13.5 & 27.0 \\
\end{array}$ & $\begin{array}{llll}88.6 & 10.3 & 26.8 \\
\end{array}$ & $\begin{array}{llll}90.5 & 10.9 & 26.1 \\
\end{array}$ & $88.3 \quad 10.42$ \\
\hline 9 & 12.025 .8 & $\begin{array}{llll}94.7 & 12.0 & 25.8 \\
\end{array}$ & $\begin{array}{llll}95.0 & 12.4 & 26.4 \\
\end{array}$ & $\begin{array}{llll}88.7 & 9.5 & 26.3 \\
\end{array}$ & $90.5 \quad 10.2 \quad 25.5$ & $\begin{array}{lll}89.1 & 9.9 & 26.0 \\
\end{array}$ \\
\hline 10 & $11.5 \quad 23.8$ & $\begin{array}{llll}93.7 & 11.5 & 23.9 \\
\end{array}$ & $\begin{array}{llll}93.9 & 11.7 & 24 \\
\end{array}$ & $9.0 \quad 24.1$ & $\begin{array}{llll}90.3 & 9.9 & 23 \\
\end{array}$ & 9.6 \\
\hline 11 & 10.723 .7 & $\begin{array}{lll}94.5 & 10.7 & 23.7 \\
\end{array}$ & $\begin{array}{llll}94.9 & 11.0 & 24 . \\
\end{array}$ & $8.4 \quad 23.8$ & $\begin{array}{lll}90.7 & 9.4 & 23.5 \\
\end{array}$ & 90.4 \\
\hline 12 & 10.022 .0 & $\begin{array}{llll}94.4 & 10.0 & 22.0 \\
\end{array}$ & $\begin{array}{lll}94.7 & 10.2 & 22.4 \\
\end{array}$ & 87.0 & $90.9 \quad 8.9 \quad 21.9$ & 90.0 \\
\hline 13 & $\begin{array}{lll}95.1 & 9.6 & 21.4 \\
\end{array}$ & $\begin{array}{llll}95.0 & 9.6 & 21.3 \\
\end{array}$ & $\begin{array}{llll}95.8 & 9.9 & 21.8 \\
\end{array}$ & 88.3 & $\begin{array}{llll}92.5 & 8.6 & 21.2 \\
\end{array}$ & 91.0 \\
\hline 14 & $\begin{array}{lll}95.3 & 9.2 & 20.0 \\
\end{array}$ & $\begin{array}{lll}95.3 & 9.2 & 20.0 \\
\end{array}$ & $\begin{array}{lll}95.5 & 9.3 & 20 .\end{array}$ & $7.2 \quad 20.5$ & \begin{tabular}{|lll}
92.9 & 8.3 & 19.8 \\
\end{tabular} & $\begin{array}{lll}91.7 & 8.1 & 20.5 \\
\end{array}$ \\
\hline 15 & \begin{tabular}{|lll}
93.9 & 8.8 & 19.3 \\
\end{tabular} & $\begin{array}{lll}93.8 & 8.8 & 19.3 \\
\end{array}$ & $\begin{array}{lll}94.4 & 9.0 & 19 .\end{array}$ & 6.919 .6 & $\begin{array}{lll}91.1 & 8.0 & 19.1 \\
\end{array}$ & $\begin{array}{lll}90.6 & 7.9 & 19.6 \\
\end{array}$ \\
\hline 20 & $\begin{array}{llll}94.4 & 7.4 & 17.0 \\
\end{array}$ & $\begin{array}{llll}94.5 & 7.4 & 17.0 \\
\end{array}$ & $\begin{array}{lll}94.7 & 7.5 & 17.3 \\
\end{array}$ & $\begin{array}{lll}87.5 & 5.9 & 17.3 \\
\end{array}$ & $\begin{array}{llll}92.5 & 6.9 & 17.1 \\
\end{array}$ & $\begin{array}{llll}91.7 & 6.8 & 17.6 \\
\end{array}$ \\
\hline 25 & $\begin{array}{llll}95.5 & 6.5 & 14.9 \\
\end{array}$ & $\begin{array}{lll}95.4 & 6.5 & 14.9 \\
\end{array}$ & $\begin{array}{lll}95.8 & 6.6 & 15 .\end{array}$ & $\begin{array}{|lll|}88.0 & 5.2 & 15.2 \\
\end{array}$ & \begin{tabular}{|lll}
93.9 & 6.2 & 14.8 \\
\end{tabular} & $\begin{array}{lll}93.3 & 6.1 & 15.1 \\
\end{array}$ \\
\hline 30 & $\begin{array}{lll}95.2 & 5.9 & 13.9 \\
\end{array}$ & $\begin{array}{lll}95.2 & 5.9 & 14.0 \\
\end{array}$ & $95.3 \quad 6.0$ & $4.7 \quad 14.0$ & $\begin{array}{llll}94.2 & 5.7 & 14.0 \\
\end{array}$ & $\begin{array}{lll}93.1 & 5.6 & 14.5 \\
\end{array}$ \\
\hline 35 & $\begin{array}{lll}95.7 & 5.5 & 12.8 \\
\end{array}$ & $\begin{array}{llll}95.8 & 5.5 & 12.8 \\
\end{array}$ & $95.7 \quad 5.6 \quad 13$. & $4.4 \quad 13.2$ & $\begin{array}{lll}95.1 & 5.3 & 12.9 \\
\end{array}$ & $\begin{array}{llll}94.3 & 5.2 & 13.2 \\
\end{array}$ \\
\hline 40 & $\begin{array}{lll}94.1 & 5.1 & 12.1 \\
\end{array}$ & $\begin{array}{lll}94.1 & 5.1 & 12.1 \\
\end{array}$ & $94.3 \quad 5.2$ & $4.1 \quad 12.6$ & $\begin{array}{lll}93.2 & 4.9 & 12.1\end{array}$ & $\begin{array}{llll}92.7 & 4.9 & 12.4 \\
\end{array}$ \\
\hline 45 & $\begin{array}{llll}94.4 & 4.8 & 11.6 \\
\end{array}$ & $\begin{array}{llll}94.5 & 4.8 & 11.6 \\
\end{array}$ & $\begin{array}{llll}94.6 & 4.8 & 11.8 \\
\end{array}$ & $\begin{array}{llll}87.7 & 3.8 & 11.7 \\
\end{array}$ & $\begin{array}{lll}93.9 & 4.6 & 11.7 \\
\end{array}$ & $\begin{array}{llll}93.7 & 4.6 & 12.1 \\
\end{array}$ \\
\hline 50 & $\begin{array}{llll}95.1 & 4.5 & 10.6 \\
\end{array}$ & $\begin{array}{llll}95.1 & 4.5 & 10.6 \\
\end{array}$ & $\begin{array}{lll}95.3 & 4.6 & 10.9 \\
\end{array}$ & $\begin{array}{lll}88.2 & 3.6 & 10.9 \\
\end{array}$ & $\begin{array}{lll}94.7 & 4.4 & 10.7 \\
\end{array}$ & $\begin{array}{llll}93.9 & 4.4 & 11.0 \\
\end{array}$ \\
\hline
\end{tabular}

\begin{tabular}{|c|c|c|c|c|c|c|}
\hline & T1 & Median T1 & Mad T1 & T3 & Median T3 & Mad T3 \\
\hline n & CC WD CV & CC WD CV & $\mathrm{CC}$ WD $\mathrm{CV}$ & $\mathrm{CC}$ WD $\mathrm{CV}$ & $\mathrm{CC}$ WD $\mathrm{CV}$ & CC WD CV \\
\hline 5 & $\begin{array}{llll}87.0 & 19.1 & 56.7 \\
\end{array}$ & $20.2 \quad 57.4$ & $\begin{array}{llll}79.5 & 14.4 & 51.7 \\
\end{array}$ & $86.7 \quad 20.1 \quad 37.5$ & $87.621 .2 \quad 38.0$ & $\begin{array}{llll}79.9 & 15.2 & 37.3 \\
\end{array}$ \\
\hline 6 & \begin{tabular}{|lll}
89.2 & 16.3 & 51.8 \\
\end{tabular} & $16.8 \quad 53.2$ & $\begin{array}{llll}81.5 & 12.4 & 46.3 \\
\end{array}$ & $\begin{array}{llll}86.5 & 19.9 & 32.7 \\
\end{array}$ & $\begin{array}{llll}87.3 & 20.5 & 33.3 \\
\end{array}$ & $\begin{array}{llll}80.5 & 15.3 & 33.0\end{array}$ \\
\hline 7 & $\begin{array}{llll}91.8 & 14.4 & 49.0 \\
\end{array}$ & $15.0 \quad 49.8$ & $\begin{array}{llll}84.6 & 11.0 & 43.2 \\
\end{array}$ & $\begin{array}{llll}89.9 & 19.8 & 31.2 \\
\end{array}$ & $90.920 .6 \quad 31.8$ & $\begin{array}{llll}84.0 & 15.2 & 32.1 \\
\end{array}$ \\
\hline 8 & $\begin{array}{llll}90.2 & 13.0 & 45.4 \\
\end{array}$ & $13.4 \quad 46.7$ & $\begin{array}{llll}84.1 & 10.0 & 39.7 \\
\end{array}$ & $\begin{array}{llll}90.1 & 19.2 & 27.7 \\
\end{array}$ & $\begin{array}{llll}90.7 & 19.8 & 28.2 \\
\end{array}$ & $84.6 \quad 14.9 \quad 28.3$ \\
\hline 9 & $\begin{array}{llll}91.9 & 11.7 & 41.1 \\
\end{array}$ & $\begin{array}{llll}92.8 & 12.1 & 42.1 \\
\end{array}$ & $\begin{array}{lll}84.3 & 9.1 & 35.6 \\
\end{array}$ & $\begin{array}{llll}90.9 & 18.7 & 26.8 \\
\end{array}$ & $\begin{array}{llll}91.5 & 19.3 & 27.4 \\
\end{array}$ & $\begin{array}{llll}84.1 & 14.6 & 27.6 \\
\end{array}$ \\
\hline 10 & $90.4 \quad 11.2 \quad 38.4$ & $\begin{array}{llll}91.1 & 11.5 & 39.2\end{array}$ & 8.733 .0 & $90.7 \quad 18.7 \quad 24.8$ & $\begin{array}{llll}91.2 & 19.2 & 25.3\end{array}$ & $\begin{array}{lllll}85.0 & 14.5 & 25.7\end{array}$ \\
\hline 11 & \begin{tabular}{|llll}
92.1 & 10.5 & 39.1 \\
\end{tabular} & $\begin{array}{ll}10.8 & 39.8 \\
\end{array}$ & 8.133 .0 & $90.6 \quad 18.3 \quad 24.2$ & $\begin{array}{llll}90.9 & 18.8 & 24.7 \\
\end{array}$ & $84.9 \quad 14.1 \quad 24.6$ \\
\hline 12 & $\begin{array}{lll}91.9 & 9.8 & 37.3 \\
\end{array}$ & $\begin{array}{lll}92.2 & 10.0 & 37.9\end{array}$ & $\begin{array}{ll}7.6 & 31.4\end{array}$ & $\begin{array}{llll}90.9 & 17.8 & 22.9\end{array}$ & $\begin{array}{llll}91.4 & 18.2 & 23.3\end{array}$ & $\begin{array}{lllll}85.0 & 13.8 & 23.5 \\
\end{array}$ \\
\hline 13 & $9.3 \quad 32.4$ & $\begin{array}{llll}93.5 & 9.5 & 32.9 \\
\end{array}$ & 85.3 & $\begin{array}{llll}92.1 & 17.7 & 22.2 \\
\end{array}$ & $92.7 \quad 18.1 \quad 22.5$ & $\begin{array}{llll}86.1 & 13.7 & 22.7 \\
\end{array}$ \\
\hline 14 & $\begin{array}{|lll|}93.3 & 8.7 & 28.3 \\
\end{array}$ & \begin{tabular}{|lll}
93.5 & 8.9 & 29.1
\end{tabular} & 85.7 & $\begin{array}{llll}92.6 & 17.3 & 20.8\end{array}$ & $\begin{array}{llll}92.9 & 17.6 & 21.2\end{array}$ & $\begin{array}{llll}86.6 & 13.5 & 21.5\end{array}$ \\
\hline 15 & 91.8 & $\begin{array}{llll}92.3 & 8.6 & 27.4 \\
\end{array}$ & 85.1 & $\begin{array}{llll}91.6 & 17.0 & 19.7 \\
\end{array}$ & $\begin{array}{llll}92.1 & 17.4 & 20.2 \\
\end{array}$ & \begin{tabular}{|llll}
85.9 & 13.3 & 20.2 \\
\end{tabular} \\
\hline 20 & $\begin{array}{lll}92.8 & 7.1 & 20.0 \\
\end{array}$ & \begin{tabular}{|lll}
93.1 & 7.2 & 20.4 \\
\end{tabular} & 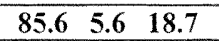 & $\begin{array}{llll}92.5 & 15.8 & 17.5 \\
\end{array}$ & $\begin{array}{llll}92.5 & 16.0 & 17.8 \\
\end{array}$ & \begin{tabular}{|l|l|l|}
86.5 & 12.4 & 17.8 \\
\end{tabular} \\
\hline 25 & 94.1 & $\begin{array}{|lll|}94.3 & 6.4 & 16.8 \\
\end{array}$ & $\begin{array}{ll}5.0 & 15.9 \\
\end{array}$ & $93.6 \quad 14.7 \quad 15.4$ & $\begin{array}{llll}93.7 & 14.9 & 15.7 \\
\end{array}$ & $\begin{array}{llll}88.8 & 11.5 & 15.9 \\
\end{array}$ \\
\hline 30 & $94.1 \quad 5.8$ & $\begin{array}{llll}94.5 & 5.8 & 15.8 \\
\end{array}$ & $\begin{array}{ll}4.5 & 14.1 \\
\end{array}$ & $\begin{array}{llll}93.4 & 13.5 & 14.3 \\
\end{array}$ & $\begin{array}{lllll}93.5 & 13.6 & 14.6\end{array}$ & $\begin{array}{llll}88.5 & 10.6 & 14.3\end{array}$ \\
\hline 35 & 94.8 & $5.4 \quad 13.6$ & $\begin{array}{ll}4.2 & 13.3 \\
\end{array}$ & $94.1 \quad 10.1 \quad 21.0$ & $94.5 \quad 10.2 \quad 21.4$ & $\begin{array}{lll}89.3 & 7.9 & 19.8 \\
\end{array}$ \\
\hline 40 & 93.5 & $\begin{array}{ll}5.0 & 13.0 \\
\end{array}$ & 85.1 & $\begin{array}{lll}94.3 & 6.7 & 12.8 \\
\end{array}$ & $\begin{array}{llll}94.4 & 6.8 & 13.1 \\
\end{array}$ & $\begin{array}{lll}89.3 & 5.3 & 13.0 \\
\end{array}$ \\
\hline 45 & 93.7 & $\begin{array}{ll}4.7 & 12.2 \\
\end{array}$ & 87.1 & $\begin{array}{lll}94.6 & 5.9 & 12.0 \\
\end{array}$ & $\begin{array}{lll}94.6 & 5.9 & 12.3\end{array}$ & $\begin{array}{lll}89.3 & 4.6 & 11.8\end{array}$ \\
\hline 50 & $\begin{array}{lll}94.5 & 4.4 \\
\end{array}$ & $\begin{array}{llll}94.5 & 4.5 & 11.1 \\
\end{array}$ & $3.5 \quad 11.0$ & $\begin{array}{lll}94.7 & 5.4 & 11.0\end{array}$ & $\begin{array}{llll}94.9 & 5.4 & 11.3\end{array}$ & $\begin{array}{lll}89.4 & 4.2 & 11.2 \\
\end{array}$ \\
\hline
\end{tabular}


Table A6: Estimated Coverage Probabilities using $\chi^{2}(8)$ with Skewness $=1(\mathrm{CC}=$ Confidence Coefficient, $\mathrm{WD}=$ Average Width, $\mathrm{CV}=$ Coefficient of Variation)

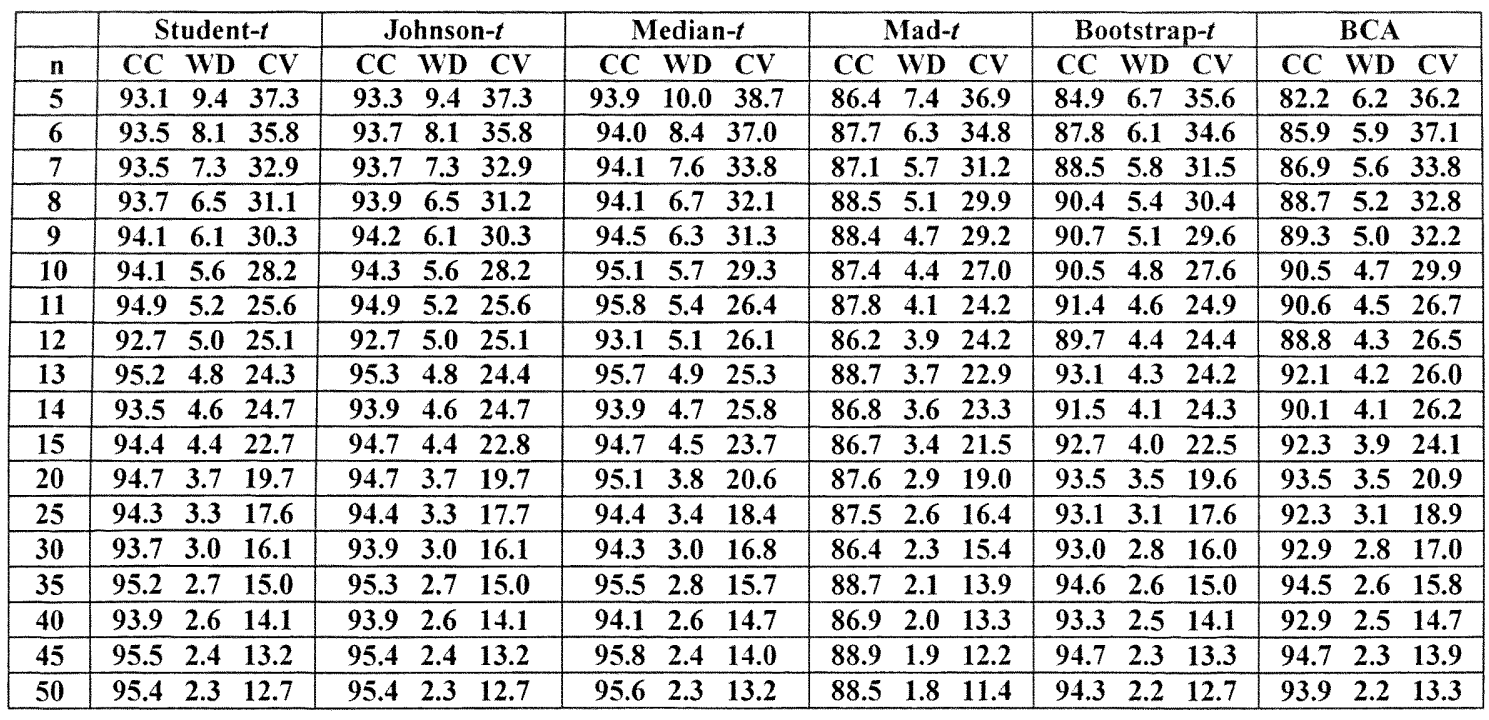

\begin{tabular}{|c|c|c|c|c|c|c|c|c|c|c|c|c|c|c|c|c|}
\hline & \multicolumn{2}{|c|}{$\mathrm{T} 1$} & \multicolumn{2}{|c|}{ Median T1 } & \multicolumn{3}{|c|}{ Mad T1 } & \multicolumn{3}{|c|}{ T3 } & \multicolumn{3}{|c|}{ Median T3 } & \multicolumn{3}{|c|}{ Mad T3 } \\
\hline n & $\mathrm{CC} \mathrm{W}$ & WD CV & $\mathrm{CC} \mathrm{V}$ & WD CV & $\mathrm{CC}$ & WD & $\mathrm{CV}$ & $\mathrm{CC}$ & WD & $\mathrm{CV}$ & $\mathrm{CC}$ & WD & $\mathrm{CV}$ & $\mathrm{CC}$ & WVD & $\mathrm{CV}$ \\
\hline 5 & 87.79. & $\begin{array}{ll}9.8 & 56.5\end{array}$ & $\begin{array}{ll}88.8 & 1 \\
\end{array}$ & $10.4 \quad 57.7$ & 80.1 & 7.4 & 51.4 & 87.3 & 9.9 & 39.2 & 88.9 & 10.5 & 40.6 & 80.1 & 7.6 & 39.4 \\
\hline 6 & 89.7 & 61.5 & 90.58 & \begin{tabular}{|l|l|}
8.9 & 63.3 \\
\end{tabular} & 82.3 & 6.5 & 55.6 & 89.1 & 9.8 & 37.5 & 89.3 & 10.2 & 38.7 & 81.8 & 7.4 & 36.0 \\
\hline 7 & 90.6 & $8.0 \quad 61.4$ & 91.5 & $8.4 \quad 62.4$ & 83.3 & 6.0 & 53.6 & 90.9 & 9.7 & 34.8 & 91.9 & 10.2 & 35.7 & 85.1 & 7.4 & 32.9 \\
\hline 8 & 91.3 & 59.0 & 91.5 & 60.3 & 83.7 & 5.3 & 50.1 & 90.8 & 9.4 & 32.9 & 91.1 & 9.7 & 34.0 & 83.7 & 7.2 & 31.6 \\
\hline 9 & 91.7 & $6.5 \quad 56.5$ & 92.1 & 57.5 & 83.9 & 4.9 & 48.5 & 91.9 & 9.3 & 32.0 & 92.6 & 9.7 & 33.1 & 85.8 & 7.1 & 30.9 \\
\hline 10 & 92.2 & 56.7 & 92.5 & 58.2 & 84.3 & 4.5 & 46.4 & 92.4 & 9.0 & 29.3 & 92.9 & 9.3 & 30.8 & 86.8 & 6.9 & 28.3 \\
\hline 11 & 92.9 & 52.1 & 93.3 & 53.1 & 85.9 & 4.3 & 43.9 & 92.7 & 8.9 & 26.4 & 93.1 & 9.2 & 27.1 & 86.9 & 6.8 & 25.5 \\
\hline 12 & 91.7 & 48.1 & 92.1 & 49.5 & 83.6 & 4.0 & 40.1 & 92.5 & 8.6 & 26.3 & 93.2 & 8.9 & 27.6 & 87.3 & 6.7 & 25.7 \\
\hline 13 & 93.9 & 49.1 & 94.2 & 5.150 .2 & 86.2 & 3.8 & 41.3 & 93.1 & 8.7 & 25.8 & 93.4 & 8.9 & 26.6 & 88.2 & 6.7 & 24.8 \\
\hline 14 & 91.9 & $4.7 \quad 47.2$ & 92.7 & 48.3 & 83.9 & 3.6 & 37.5 & 93.1 & 8.5 & 26.1 & 93.5 & 8.7 & 27.2 & 87.6 & 6.5 & 24.8 \\
\hline 15 & 93.5 & $4.5 \quad 42.3$ & 93.9 & $4.6 \quad 43.0$ & 85.5 & 3.4 & 34.3 & 92.9 & 8.3 & 23.7 & 93.5 & 8.6 & 24.7 & 87.1 & 6.4 & 23.1 \\
\hline 20 & 94.4 & $3.8 \quad 33.5$ & 94.5 & 34.2 & 85.9 & 2.9 & 27.4 & 93.3 & 7.9 & 20.4 & 94.1 & 8.1 & 21.5 & 88.1 & 6.1 & 19.9 \\
\hline 25 & 93.6 & $\begin{array}{ll}3.3 & 27.0\end{array}$ & 94.0 & 3.427 .6 & 86.3 & 2.5 & 20.7 & 94.3 & 7.3 & 18.4 & 94.9 & 7.5 & 19.3 & 89.0 & 5.7 & 17.3 \\
\hline 30 & 93.6 & $2.9 \quad 21.2$ & 94.3 & 21.8 & 85.6 & 2.3 & 17.9 & 95.4 & 6.7 & 17.2 & 95.5 & 6.8 & 17.9 & 90.3 & 5.2 & 16.5 \\
\hline 35 & 94.9 & $\begin{array}{ll}2.7 & 19.6\end{array}$ & 95.2 & $2.8 \quad 20.1$ & 88.5 & 2.1 & 15.4 & 95.7 & 5.5 & 21.0 & 96.1 & 5.6 & 21.7 & 91.1 & 4.2 & 19.0 \\
\hline 40 & 93.9 & $\begin{array}{ll}2.5 & 17.1 \\
\end{array}$ & 94.0 & 17.6 & 86.7 & 2.0 & 14.4 & 95.3 & 3.4 & 15.1 & 95.5 & 3.4 & 15.8 & 89.7 & 2.6 & 13.8 \\
\hline 45 & 95.1 & $2.4 \quad 15.6$ & 95.52 & 16.2 & 88.7 & 1.9 & 13.4 & 95.1 & 3.0 & 13.9 & 95.3 & 3.0 & 14.6 & 90.8 & 2.3 & 12.7 \\
\hline 50 & $94.8 \quad 2$ & $2.3 \quad 14.0$ & 95.12 & $2.3 \quad 14.5$ & 87.5 & 1.8 & 11.9 & 95.1 & 2.7 & 13.3 & 95.3 & 2.7 & 13.9 & 89.3 & 2.1 & 11.9 \\
\hline
\end{tabular}


Table A7: Estimated Coverage Probabilities using $\chi^{2}(2)$ with Skewness $=2(\mathrm{CC}=$ Confidence Coefficient, $\mathrm{WD}=$ Average Width, $\mathrm{CV}=$ Coefficient of Variation)

\begin{tabular}{|c|c|c|c|c|c|c|c|c|c|c|c|c|c|c|c|c|}
\hline & Student- $t$ & \multicolumn{3}{|c|}{ Johnson-t } & \multicolumn{3}{|c|}{ Median- $t$} & \multicolumn{3}{|c|}{ Mad- $t$} & \multicolumn{3}{|c|}{ Bootstrap-t } & \multicolumn{3}{|c|}{$\mathrm{BCA}$} \\
\hline n & CC WD CV & $\mathrm{CC}$ & WD & $\mathrm{CV}$ & $\mathrm{CC}$ & WD & $\mathrm{CV}$ & $\mathrm{CC}$ & WD & $\mathrm{CV}$ & $\mathrm{CC}$ & WD & $\mathrm{CV}$ & $\mathrm{CC}$ & WD & $\mathrm{CV}$ \\
\hline 5 & $\begin{array}{|lll|}88.8 & 4.7 & 52.7 \\
\end{array}$ & 89.0 & 4.7 & 52.8 & 89.9 & 5.0 & 55.0 & 81.9 & 3.7 & 49.3 & 81.3 & 3.4 & 49.2 & 79.9 & 3.2 & 51.8 \\
\hline 6 & $\begin{array}{lll}89.5 & 4.0 & 46.6 \\
\end{array}$ & 89.7 & 4.0 & 46.7 & 90.2 & 4.2 & 48.8 & 83.2 & 3.1 & 43.3 & 83.9 & 3.0 & 43.7 & 82.7 & 3.0 & 48.4 \\
\hline 7 & $\begin{array}{|lll|}89.3 & 3.6 & 43.9 \\
\end{array}$ & 89.7 & 3.6 & 44.0 & 90.1 & 3.8 & 45.5 & 83.5 & 2.8 & 40.3 & 85.1 & 2.8 & 41.4 & 85.0 & 2.8 & 46.2 \\
\hline 8 & $\begin{array}{lll}90.2 & 3.3 & 43.2 \\
\end{array}$ & 90.3 & 3.3 & 43.3 & 90.9 & 3.4 & 44.9 & 83.1 & 2.5 & 39.0 & 86.6 & 2.7 & 41.3 & 85.9 & 2.7 & 46.2 \\
\hline 9 & $\begin{array}{lll}89.4 & 3.0 & 39.7 \\
\end{array}$ & 89.9 & 3.0 & 39.8 & 90.7 & 3.2 & 41.3 & 81.9 & 2.3 & 35.2 & 85.6 & 2.5 & 37.5 & 86.2 & 2.5 & 42.5 \\
\hline 10 & $\begin{array}{lll}89.6 & 2.8 & 37.2 \\
\end{array}$ & 89.9 & 2.8 & 37.3 & 90.3 & 3.0 & 38.9 & 83.7 & 2.2 & 33.5 & 87.6 & 2.4 & 35.7 & 88.3 & 2.4 & 41.1 \\
\hline 11 & $2.6 \quad 34.6$ & 90.7 & 2.6 & 34.8 & 91.1 & 2.7 & 36.1 & 83.9 & 2.0 & 31.0 & 88.4 & 2.3 & 33.7 & 88.4 & 2.3 & 38.7 \\
\hline 12 & 34.8 & 90.3 & 2.5 & 34.9 & 90.7 & 2.6 & 36.4 & 83.5 & 1.9 & 30.1 & 88.6 & 2.2 & 33.8 & 88.7 & 2.2 & 38.6 \\
\hline 13 & $2.4 \quad 32.8$ & 91.3 & 2.4 & 32.8 & 91.9 & 2.5 & 34.0 & 84.1 & 1.8 & 28.9 & 89.2 & 2.1 & 31.8 & 89.1 & 2.1 & 36.7 \\
\hline 14 & 91.2 & 91.3 & 2.3 & 32.8 & 91.7 & 2.4 & 34.3 & 84.3 & 1.7 & 28.3 & 89.6 & 2.0 & 31.6 & 88.4 & 2.1 & 36.0 \\
\hline 15 & 91.7 & 92.2 & 2.2 & 31.5 & 92.6 & 2.3 & 32.8 & 85.7 & 1.7 & 27.4 & 90.5 & 2.0 & 30.6 & 90.8 & 2.0 & 34.0 \\
\hline 20 & 91.3 & 91.7 & 1.8 & 27.9 & 92.3 & 1.9 & 29.3 & 83.5 & 1.4 & 23.7 & 90.6 & 1.7 & 27.3 & 91.1 & 1.7 & 30.7 \\
\hline 25 & 92.2 & 92.7 & 1.6 & 25.3 & 93.2 & 1.7 & 26.2 & 84.0 & 1.2 & 20.2 & 91.5 & 1.5 & 24.9 & 91.7 & 1.6 & 28.3 \\
\hline 30 & 92.0 & 92.5 & 1.5 & 23.6 & 93.3 & 1.6 & 24.4 & 84.1 & 1.1 & 18.3 & 91.4 & 1.4 & 23.0 & 91.7 & 1.4 & 26.0 \\
\hline 35 & 93.8 & 93.9 & 1.3 & 21.6 & 94.7 & 1.4 & 22.4 & 84.5 & 1.0 & 17.0 & 93.0 & 1.3 & 21.4 & 93.2 & 1.3 & 23.3 \\
\hline 40 & 93.9 & 94.0 & 1.3 & 19.7 & 94.7 & 1.3 & 20.4 & 85.5 & 1.0 & 15.6 & 93.5 & 1.2 & 19.6 & 92.7 & 1.2 & 21.4 \\
\hline 45 & $94.3 \quad 1.2$ & 94.5 & 1.2 & 19.5 & 95.1 & 1.3 & 20.2 & 85.3 & 0.9 & 14.9 & 93.9 & 1.2 & 19.4 & 94.3 & 1.2 & 21.4 \\
\hline 50 & $\begin{array}{lll}93.1 & 1.1 & 17.8 \\
\end{array}$ & 93.1 & 1.1 & 17.8 & 94.1 & 1.2 & 18.5 & 84.6 & 0.8 & 14.3 & 92.3 & 1.1 & 17.7 & 92.4 & 1.1 & 19.2 \\
\hline
\end{tabular}

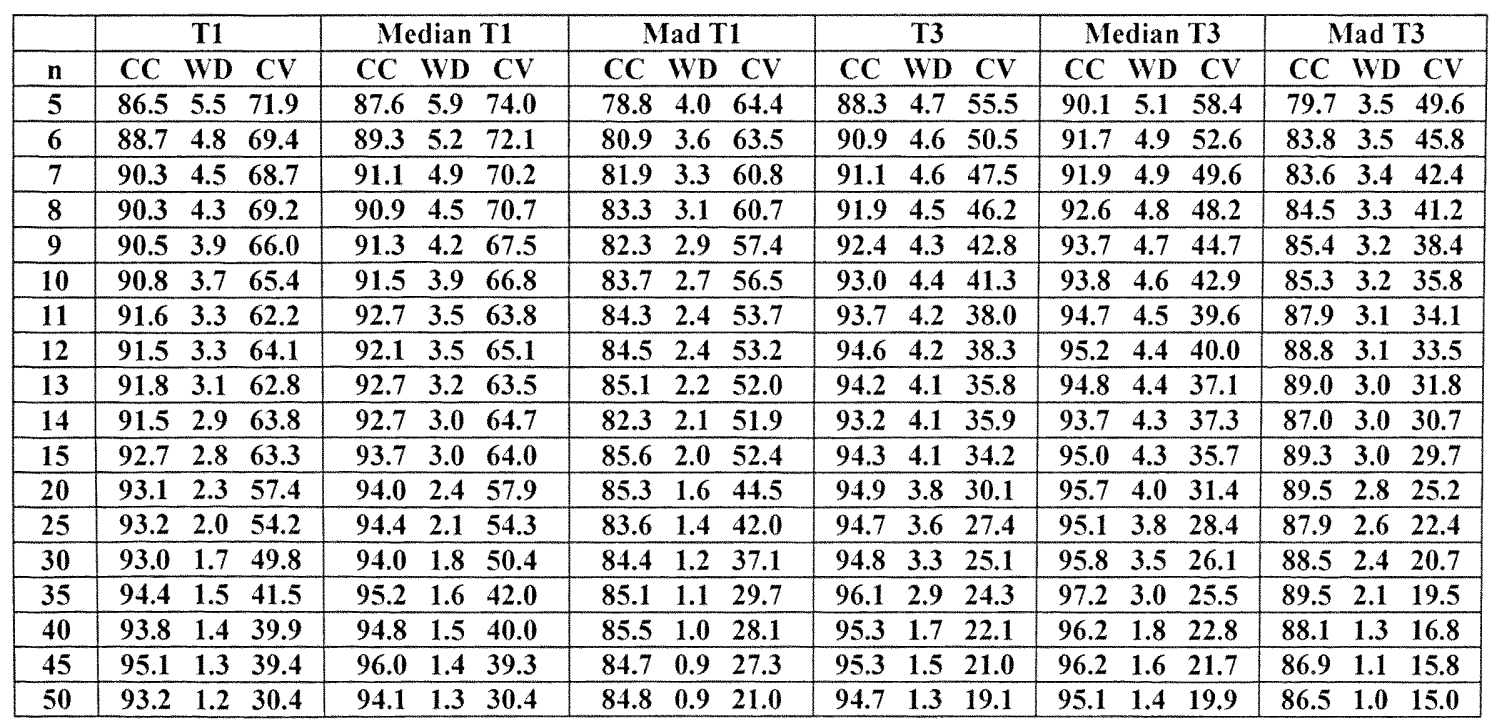


Table A8: Estimated Coverage Probabilities using $\chi^{2}(.5)$ with Skewness $=4(\mathrm{CC}=$ Confidence Coefficient, WD $=$ Average Width, $\mathrm{CV}=$ Coefficient of Variation)

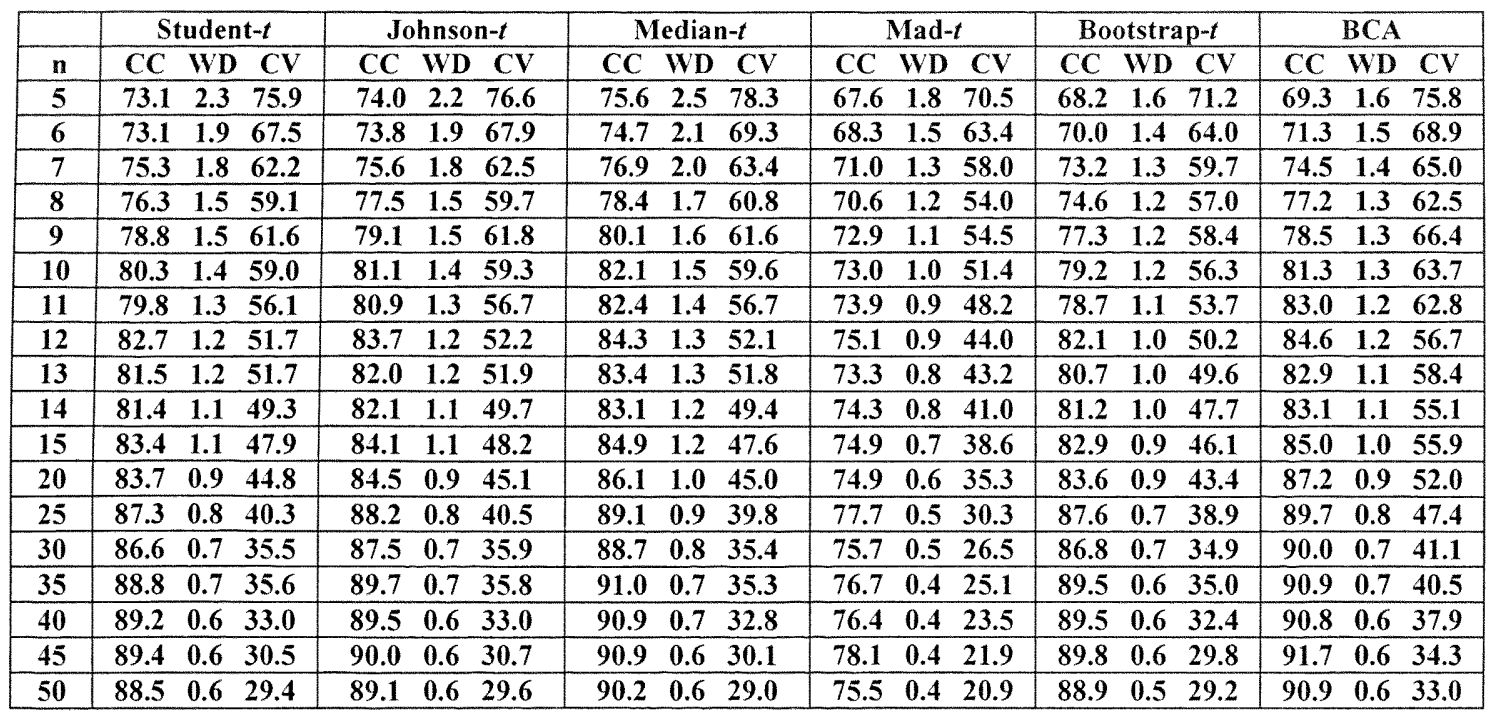

\begin{tabular}{|c|c|c|c|c|c|c|c|c|c|c|c|c|c|c|c|c|c|}
\hline & \multicolumn{2}{|c|}{$\mathrm{T} 1$} & \multicolumn{3}{|c|}{ Median T1 } & \multicolumn{3}{|c|}{ Mad T1 } & \multicolumn{3}{|c|}{ T3 } & \multicolumn{3}{|c|}{ Median T3 } & \multicolumn{3}{|c|}{ Mad T3 } \\
\hline n & $\mathrm{CC} \mathrm{V}$ & WD CV & $\mathrm{CC} \mathrm{W}$ & WD & $\mathrm{CV}$ & $\mathrm{CC}$ & WD & $\mathrm{CV}$ & $\mathrm{CC}$ & WD & $\mathrm{CV}$ & $\mathrm{CC}$ & WD & $\mathrm{CV}$ & $\mathrm{CC}$ & WD & $\mathrm{CV}$ \\
\hline 5 & 80.62 & 2.789 .6 & 82.33 & 3.09 & 91.7 & 72.9 & 1.9 & 79.4 & 78.1 & 2.2 & 81.7 & 80.5 & 2.5 & 85.3 & 69.3 & 1.5 & 69.8 \\
\hline 6 & 82.6 & $2.5 \quad 82.2$ & 4.1 & 2.7 & 84.1 & 74.3 & 1.7 & 70.5 & 80.1 & 2.1 & 74.8 & 82.1 & 2.3 & 77.7 & 70.3 & 1.4 & 64.4 \\
\hline 7 & 84.4 & 2.475 .8 & 86.3 & 2.7 & 77.5 & 76.1 & 1.7 & 66.0 & 84.6 & 2.1 & 70.6 & 87.3 & 2.3 & 72.6 & 74.3 & 1.4 & 58.9 \\
\hline 8 & 87.7 & $\begin{array}{ll}2.2 & 72.8 \\
\end{array}$ & 89.3 & 2.4 & 74.9 & 79.9 & 1.5 & 62.5 & 88.3 & 2.0 & 67.2 & 90.1 & 2.2 & 69.5 & 78.1 & 1.3 & 54.0 \\
\hline 9 & 87.9 & 75.0 & 89.1 & 2.4 & 75.2 & 77.3 & 1.4 & 59.4 & 87.6 & 2.0 & 69.6 & 89.5 & 2.3 & 70.0 & 75.9 & 1.3 & 50.2 \\
\hline 10 & 90.5 & 73.4 & 91.9 & 2.4 & 74.6 & 80.7 & 1.4 & 60.5 & 90.8 & 2.0 & 65.9 & 93.3 & 2.3 & 67.4 & 80.7 & 1.3 & 52.2 \\
\hline 11 & 90.5 & 71.0 & 92.1 & 2.3 & 71.3 & 81.3 & 1.3 & 57.2 & 91.9 & 2.0 & 64.6 & 94.5 & 2.2 & 65.0 & 82.5 & 1.3 & 49.0 \\
\hline 12 & 91.3 & 65.7 & 92.2 & 2.2 & 66.4 & 81.6 & 1.3 & 53.6 & 93.4 & 2.0 & 59.0 & 94.8 & 2.2 & 59.6 & 83.7 & 1.3 & 46.2 \\
\hline 13 & 91.1 & $1.8 \quad 67.5$ & 92.9 & 2.0 & 67.8 & 80.3 & 1.2 & 53.8 & 93.9 & 1.9 & 60.0 & 95.8 & 2.1 & 59.8 & 83.5 & 1.2 & 44.9 \\
\hline 14 & 91.3 & 64.1 & 93.2 & 2.0 & 65.2 & 81.1 & 1.2 & 52.1 & 93.1 & 1.9 & 56.3 & 94.9 & 2.1 & 56.7 & 83.5 & 1.2 & 44.7 \\
\hline 15 & 92.2 & $1.7 \quad 64.1$ & 94.11 & 1.9 & 64.1 & 81.8 & 1.1 & 50.9 & 94.8 & 1.9 & 56.0 & 96.6 & 2.1 & 55.7 & 84.3 & 1.2 & 41.8 \\
\hline 20 & 93.3 & $1.5 \quad 63.8$ & 94.8 & 1.7 & 63.7 & 80.1 & 0.9 & 47.6 & 95.7 & 1.8 & 51.8 & 97.6 & 2.0 & 51.4 & 85.1 & 1.1 & 38.6 \\
\hline 25 & 93.8 & $1.3 \quad 60.1$ & 95.5 & 1.5 & 59.0 & 83.4 & 0.8 & 46.1 & 96.9 & 1.7 & 45.8 & 97.9 & 1.9 & 44.7 & 88.3 & 1.0 & 34.0 \\
\hline 30 & 94.1 & 1.258 .0 & 96.1 & 1.3 & 57.5 & 82.4 & 0.7 & 44.8 & 97.2 & 1.6 & 41.5 & 98.4 & 1.7 & 40.8 & 87.6 & 1.0 & 31.9 \\
\hline 35 & 93.7 & 1.160 .8 & 95.8 & 1.1 & 60.0 & 83.1 & 0.6 & 45.7 & 97.2 & 1.4 & 40.4 & 98.3 & 1.6 & 39.5 & 88.9 & 0.9 & 29.6 \\
\hline 40 & 93.5 & $1.0 \quad 59.3$ & 95.9 & 1.0 & 57.9 & 80.5 & 0.6 & 43.7 & 94.5 & 0.9 & 38.6 & 96.5 & 0.9 & 37.5 & 82.5 & 0.5 & 25.6 \\
\hline 45 & 94.8 & \begin{tabular}{|ll}
0.9 & 57.3 \\
\end{tabular} & 96.7 & 1.0 & 56.4 & 81.7 & 0.5 & 43.1 & 95.9 & 0.7 & 33.9 & 97.3 & 0.8 & 33.3 & 81.7 & 0.5 & 22.4 \\
\hline 50 & 94.10 & $\begin{array}{ll}0.8 \quad 57.2 \\
\end{array}$ & 95.70 & 0.95 & 55.9 & 79.7 & 0.5 & 41.5 & 94.5 & 0.7 & 31.8 & 96.3 & 0.7 & 31.5 & 79.7 & 0.4 & 21.3 \\
\hline
\end{tabular}


Table A9: Estimated Coverage Probabilities using Gamma(4, 2.5) with Skewness $=1$ including Median and Mad Bootstrap- $t(\mathrm{CC}=$ Confidence Coefficient, $\mathrm{WD}=$ Average Width, $\mathrm{CV}=$ Coefficient of Variation)

\begin{tabular}{|c|c|c|c|c|c|c|c|c|c|c|c|}
\hline & \multicolumn{2}{|c|}{ Student- $t$} & \multicolumn{3}{|c|}{ Johnson- $t$} & \multicolumn{3}{|c|}{ Median- $t$} & \multicolumn{3}{|c|}{ Mad- $t$} \\
\hline n & $\mathrm{CC} \quad \mathrm{WD}$ & $\mathrm{CV}$ & $\mathrm{CC}$ & WD & $\mathrm{CV}$ & $\mathrm{CC}$ & WD & $\mathrm{CV}$ & $\mathrm{CC}$ & WD & $\mathrm{CV}$ \\
\hline 5 & 93.411 .9 & 39.5 & 93.3 & 11.9 & 39.5 & 94.1 & 12.6 & 41.0 & 89.3 & 9.2 & 38.7 \\
\hline 6 & $93.0 \quad 10.0$ & 35.7 & 92.9 & 10.0 & 35.7 & 93.5 & 10.3 & 36.8 & 86.4 & 7.8 & 35.1 \\
\hline 7 & $93.4 \quad 8.9$ & 33.7 & 93.6 & 8.9 & 33.8 & 93.7 & 9.3 & 35.1 & 87.1 & 6.9 & 33.1 \\
\hline 8 & $\begin{array}{lll}93.1 & 8.1 \\
\end{array}$ & 31.6 & 93.4 & 8.1 & 31.7 & 93.6 & 8.4 & 32.8 & 87.4 & 6.3 & 30.6 \\
\hline 9 & $\begin{array}{ll}93.3 & 7.3 \\
\end{array}$ & 29.6 & 93.4 & 7.3 & 29.6 & 93.9 & 7.6 & 30.6 & 86.9 & 5.7 & 28.7 \\
\hline 10 & $93.4 \quad 7.0$ & 27.4 & 93.4 & 7.0 & 27.4 & 93.7 & 7.2 & 28.4 & 87.3 & 5.5 & 26.3 \\
\hline 11 & $92.6 \quad 6.6$ & 26.7 & 92.6 & 6.6 & 26.7 & 93.1 & 6.8 & 27.7 & 86.2 & 5.1 & 25.8 \\
\hline 12 & $94.6 \quad 6.2$ & 26.1 & 94.7 & 6.2 & 26.1 & 94.9 & 6.4 & 27.0 & 87.5 & 4.9 & 24.7 \\
\hline 13 & $94.7 \quad 6.0$ & 24.5 & 94.8 & 6.0 & 24.6 & 95.1 & 6.2 & 25.5 & 87.5 & 4.7 & 23.2 \\
\hline 14 & $\begin{array}{ll}93.7 & 5.7 \\
\end{array}$ & 23.7 & 93.6 & 5.7 & 23.7 & 93.8 & 5.8 & 24.7 & 86.1 & 4.5 & 22.7 \\
\hline 15 & $93.7 \quad 5.4$ & 23.0 & 93.9 & 5.4 & 23.0 & 94.1 & 5.6 & 23.8 & 86.6 & 4.3 & 21.6 \\
\hline 20 & $94.9 \quad 4.6$ & 19.6 & 94.9 & 4.6 & 19.6 & 95.1 & 4.7 & 20.5 & 88.5 & 3.6 & 18.3 \\
\hline 25 & $94.5 \quad 4.1$ & 17.8 & 94.5 & 4.1 & 17.9 & 94.7 & 4.2 & 18.6 & 88.3 & 3.2 & 16.9 \\
\hline 30 & $94.6 \quad 3.7$ & 15.7 & 94.9 & 3.7 & 15.7 & 95.2 & 3.8 & 16.5 & 88.1 & 2.9 & 15.2 \\
\hline 35 & 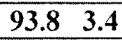 & 15.1 & 93.7 & 3.4 & 15.1 & 94.3 & 3.5 & 15.8 & 87.3 & 2.7 & 14.0 \\
\hline 40 & $94.9 \quad 3.2$ & 14.1 & 95.1 & 3.2 & 14.1 & 95.3 & 3.2 & 14.7 & 88.7 & 2.5 & 13.1 \\
\hline 45 & $94.5 \quad 3.0$ & 13.7 & 94.4 & 3.0 & 13.7 & 94.8 & 3.0 & 14.2 & 88.0 & 2.3 & 12.4 \\
\hline 50 & $94.8 \quad 2.8$ & 12.7 & 94.8 & 2.8 & 12.7 & 95.1 & 2.9 & 13.2 & 86.8 & 2.2 & 11.6 \\
\hline
\end{tabular}

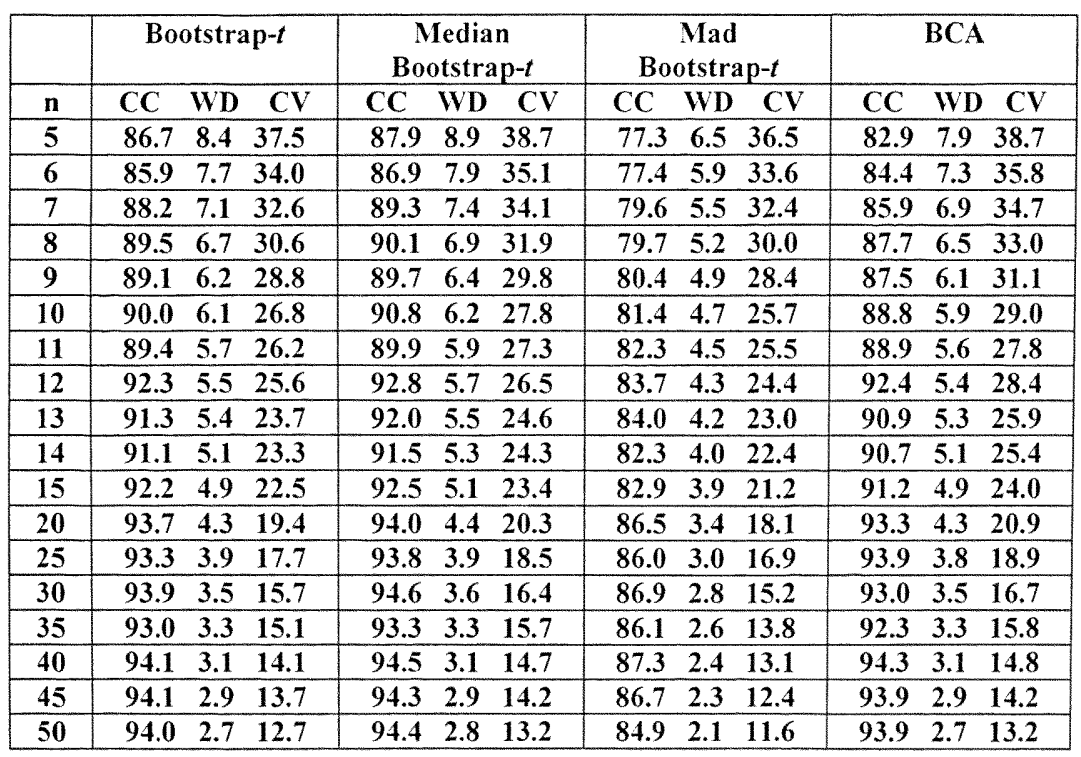




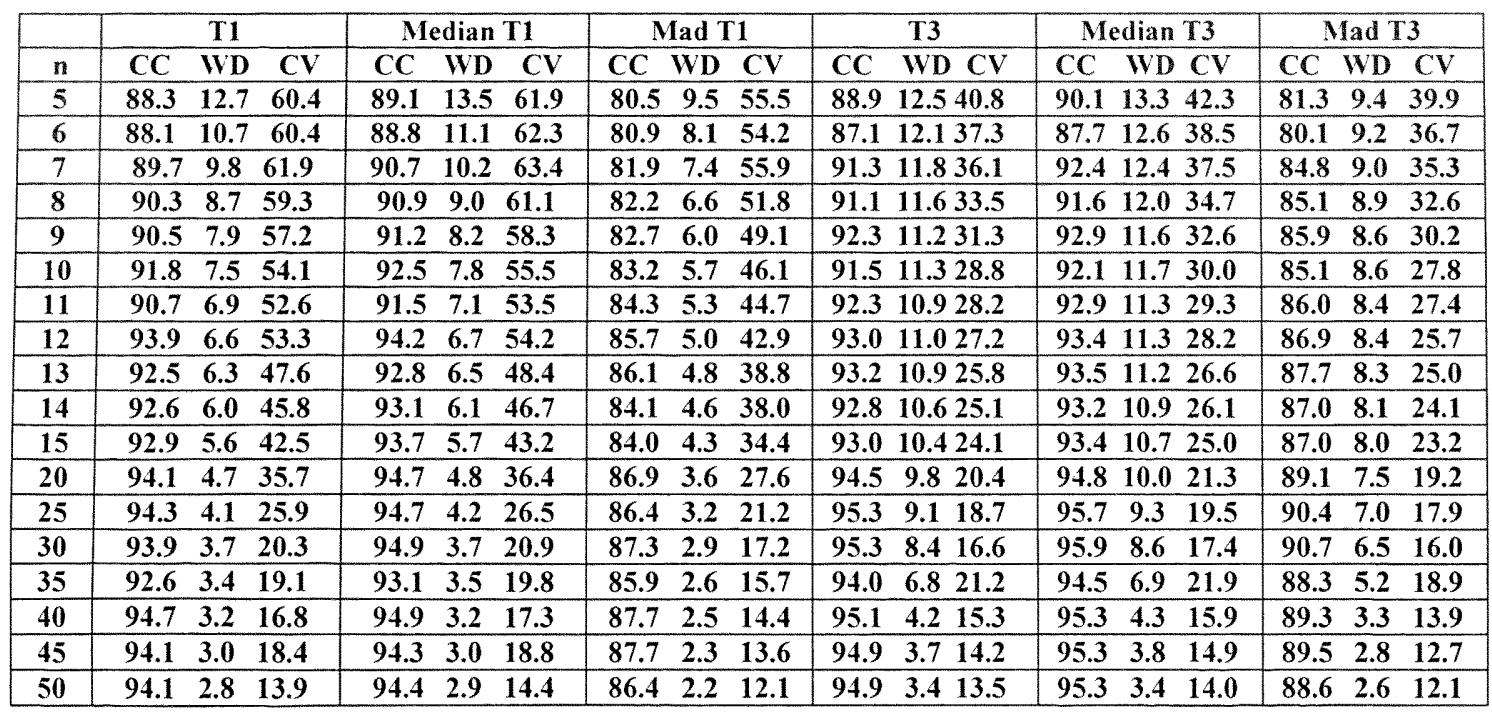


Table A10: Estimated Coverage Probabilities using Gamma $(.25,40)$ with Skewness $=4$ including Median and Mad Bootstrap- $t(\mathrm{CC}=$ Confidence Coefficient, $\mathrm{WD}=$ Average Width, $\mathrm{CV}=$ Coefficient of Variation)

\begin{tabular}{|c|ccc|ccc|ccc|ccc|}
\hline & \multicolumn{3}{|c|}{ Student- } & \multicolumn{3}{|c|}{ Johnson- $t$} & \multicolumn{3}{c|}{ Median- $t$} & \multicolumn{4}{c|}{ Mad- $t$} \\
\hline$n$ & CC & WD & CV & CC & WD & CV & CC & WD & CV & CC & WD & $C V$ \\
\hline 5 & 73.7 & 45.6 & 72.1 & 74.6 & 45.2 & 72.8 & 75.5 & 50.4 & 73.6 & 68.7 & 35.8 & 67.3 \\
\hline 6 & 73.9 & 39.0 & 70.0 & 74.9 & 38.6 & 70.6 & 76.4 & 42.0 & 72.9 & 68.9 & 30.1 & 65.1 \\
\hline 7 & 76.4 & 35.7 & 66.2 & 77.3 & 35.4 & 66.8 & 78.3 & 39.1 & 67.3 & 70.6 & 27.1 & 67.5 \\
\hline 8 & 77.0 & 32.2 & 66.8 & 77.5 & 32.1 & 67.0 & 78.8 & 35.0 & 38.3 & 70.9 & 24.2 & 60.3 \\
\hline 9 & 81.1 & 29.2 & 57.5 & 81.8 & 29.1 & 57.9 & 82.9 & 31.9 & 58.2 & 72.5 & 21.7 & 50.3 \\
\hline 10 & 80.3 & 27.2 & 55.5 & 81.2 & 27.0 & 55.9 & 82.2 & 29.5 & 56.3 & 73.4 & 20.0 & 47.5 \\
\hline 11 & 80.2 & 25.8 & 52.9 & 81.0 & 25.7 & 53.3 & 82.6 & 28.0 & 53.4 & 73.8 & 18.7 & 46.2 \\
\hline 12 & 82.1 & 24.4 & 51.2 & 82.9 & 24.3 & 51.5 & 83.9 & 26.6 & 51.8 & 73.7 & 17.8 & 44.0 \\
\hline 13 & 80.3 & 23.2 & 49.7 & 81.7 & 22.9 & 50.3 & 82.9 & 25.1 & 50.7 & 72.5 & 16.7 & 43.4 \\
\hline 14 & 81.8 & 22.2 & 50.6 & 82.5 & 22.1 & 50.9 & 83.4 & 24.1 & 50.4 & 73.1 & 15.6 & 40.7 \\
\hline 15 & 82.5 & 21.8 & 49.5 & 83.4 & 21.6 & 50.0 & 84.4 & 23.6 & 49.6 & 75.5 & 15.1 & 41.1 \\
\hline 20 & 86.2 & 18.6 & 43.5 & 86.5 & 18.5 & 43.8 & 87.5 & 20.1 & 43.3 & 77.1 & 12.5 & 33.6 \\
\hline 25 & 84.9 & 16.3 & 39.9 & 86.1 & 16.2 & 40.1 & 87.5 & 17.6 & 40.3 & 74.3 & 10.9 & 30.3 \\
\hline 30 & 87.0 & 14.9 & 37.6 & 87.5 & 14.8 & 37.8 & 88.5 & 16.1 & 37.3 & 74.5 & 9.7 & 26.8 \\
\hline 35 & 86.1 & 13.5 & 34.8 & 86.7 & 13.5 & 34.8 & 88.2 & 14.7 & 34.0 & 73.9 & 8.8 & 23.9 \\
\hline 40 & 89.3 & 12.7 & 33.1 & 89.7 & 12.7 & 33.1 & 90.8 & 13.8 & 32.6 & 76.9 & 8.2 & 23.2 \\
\hline 45 & 87.7 & 11.8 & 31.4 & 88.5 & 11.8 & 31.4 & 89.7 & 12.8 & 30.5 & 75.9 & 7.6 & 22.4 \\
\hline 50 & 90.5 & 11.1 & 29.7 & 91.0 & 11.1 & 29.7 & 91.6 & 12.1 & 28.9 & 76.5 & 7.1 & 19.7 \\
\hline
\end{tabular}

\begin{tabular}{|c|c|c|c|c|c|c|c|c|c|c|c|}
\hline & \multicolumn{2}{|c|}{ Bootstrap- $t$} & \multicolumn{3}{|c|}{$\begin{array}{c}\text { Median } \\
\text { Bootstrap- } t\end{array}$} & \multicolumn{3}{|c|}{$\begin{array}{c}\text { Mad } \\
\text { Bootstrap- } t\end{array}$} & \multicolumn{3}{|c|}{ BCA } \\
\hline $\mathbf{n}$ & $\mathrm{CC}$ & WD CV & $\mathrm{CC}$ & WD & $\mathrm{CV}$ & $\mathrm{CC}$ & WD & CV & $\mathrm{CC}$ & IVD & $\mathrm{CV}$ \\
\hline 5 & 69.1 & $31.5 \quad 67.3$ & 70.9 & 34.9 & 68.8 & 61.9 & 23.6 & 60.6 & 69.7 & 31.0 & 71.6 \\
\hline 6 & 70.7 & \begin{tabular}{|ll}
28.5 & 66.7 \\
\end{tabular} & 72.2 & 31.0 & 68.9 & 62.4 & 21.3 & 59.9 & 72.9 & 29.8 & 73.0 \\
\hline 7 & 73.3 & $26.9 \quad 60.1$ & 75.5 & 29.7 & 63.9 & 64.8 & 19.3 & 53.5 & 75.2 & 28.9 & 70.0 \\
\hline 8 & 74.8 & \begin{tabular}{|l|l|}
25.4 & 63.9 \\
\end{tabular} & 76.5 & 27.7 & 65.3 & 64.8 & 17.7 & 47.5 & 76.1 & 26.8 & 65.8 \\
\hline 9 & 78.8 & $23.7 \quad 54.9$ & 81.3 & 25.9 & 56.3 & 68.0 & 16.9 & 45.8 & 81.5 & 25.5 & 62.5 \\
\hline 10 & 78.4 & $22.5 \quad 52.4$ & 80.7 & 24.5 & 54.3 & 68.6 & 15.9 & 43.4 & 81.5 & 24.4 & 61.1 \\
\hline 11 & 78.9 & $21.9 \quad 50.7$ & 81.5 & 23.8 & 51.5 & 68.8 & 15.0 & 41.7 & 82.3 & 23.6 & 59.0 \\
\hline 12 & 81.3 & $20.9 \quad 49.8$ & 83.3 & 22.8 & 50.6 & 69.9 & 14.6 & 40.1 & 84.1 & 22.8 & 57.3 \\
\hline 13 & 79.1 & $20.1 \quad 48.5$ & 82.9 & 21.8 & 49.9 & 68.2 & 13.9 & 39.2 & 83.5 & 21.9 & 55.9 \\
\hline 14 & 80.9 & $19.2 \quad 47.5$ & 83.0 & 21.1 & 48.6 & 69.9 & 13.1 & 36.2 & 83.7 & 21.3 & 57.8 \\
\hline 15 & 81.9 & $19.2 \quad 47.9$ & 84.3 & 20.9 & 48.3 & 71.6 & 12.7 & 35.4 & 85.2 & 20.9 & 56.9 \\
\hline 20 & 85.5 & $16.8 \quad 42.3$ & 87.7 & 18.3 & 42.6 & 73.4 & 11.0 & 30.0 & 87.9 & 18.4 & 50.5 \\
\hline 25 & 85.7 & $15.0 \quad 39.3$ & 87.9 & 16.3 & 39.3 & 71.2 & 9.7 & 27.8 & 88.1 & 16.4 & 45.7 \\
\hline 30 & 87.1 & $13.9 \quad 36.7$ & 89.1 & 15.0 & 37.3 & 71.0 & 8.8 & 25.0 & 88.7 & 14.9 & 41.6 \\
\hline 35 & 86.3 & $12.7 \quad 33.9$ & 88.8 & 13.8 & 34.1 & 71.9 & 8.1 & 22.2 & 89.2 & 13.7 & 39.4 \\
\hline 40 & 89.6 & $12.1 \quad 32.2$ & 91.3 & 13.1 & 32.1 & 73.7 & 7.6 & 21.1 & 90.8 & 12.8 & 37.5 \\
\hline 45 & 88.3 & $11.3 \quad 31.0$ & 90.1 & 12.2 & 30.3 & 73.6 & 7.1 & 21.1 & 90.0 & 11.9 & 36.1 \\
\hline 50 & 90.7 & $10.6 \quad 29.2$ & 92.4 & 11.6 & 28.4 & 74.7 & 6.7 & 19.4 & 91.2 & 11.3 & 31.9 \\
\hline
\end{tabular}




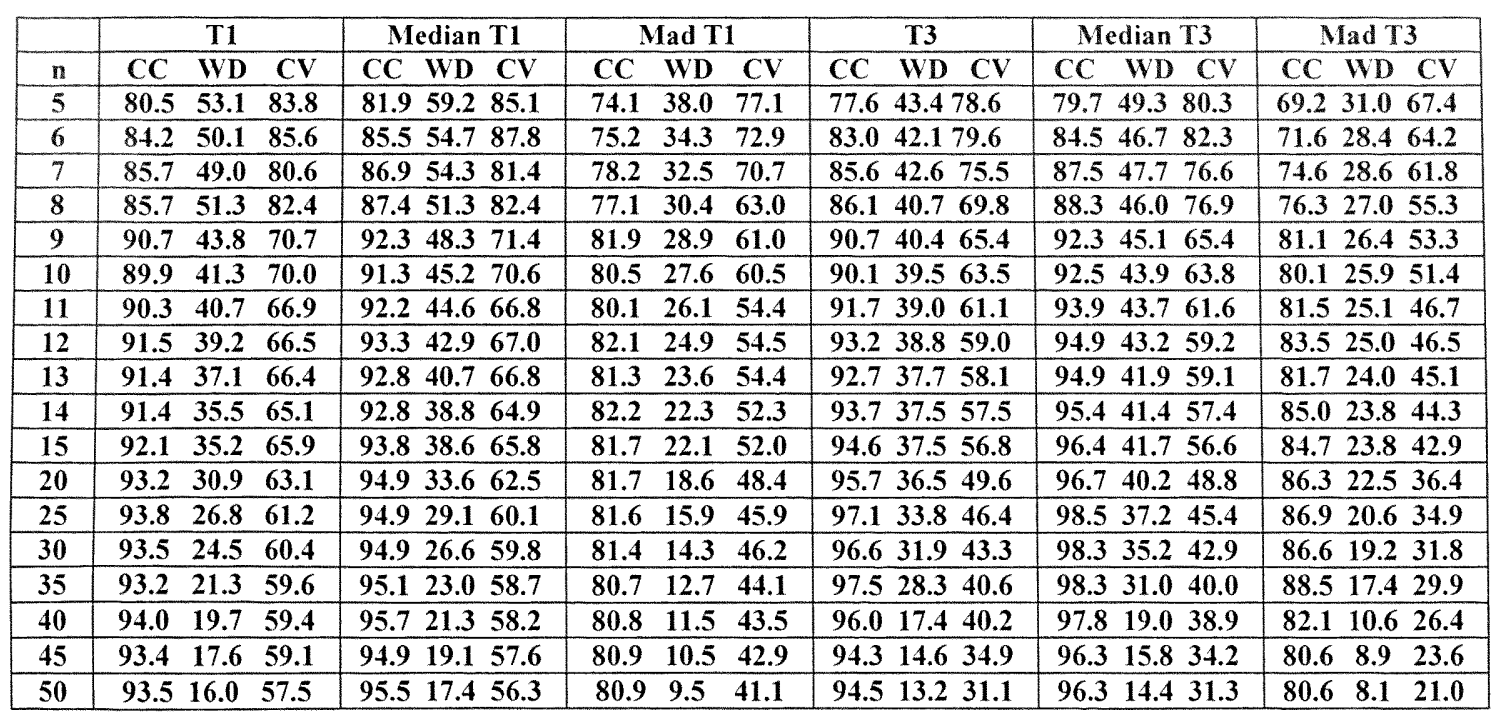


Table A11: Estimated Coverage Probabilities using $\log$ Normal $(2.25, .314)$ with Skewness $=1$ including Median and Mad Bootstrap- $t(\mathrm{CC}=$ Confidence Coefficient, $\mathrm{WD}=$ Average Width, $\mathrm{CV}=$ Coefficient of Variation)

\begin{tabular}{|c|ccc|ccc|ccc|ccc|}
\hline & \multicolumn{3}{|c|}{ Student- } & \multicolumn{3}{|c|}{ Johnson- } & \multicolumn{3}{|c|}{ Median- } & \multicolumn{4}{c|}{ Mad- $t$} \\
\hline$n$ & CC & WD & CV & \multicolumn{2}{|c|}{ CC } & WD & CV & \multicolumn{2}{|c|}{ CC } & WD & CV & \multicolumn{3}{|c|}{ CC } & WD & CV \\
\hline 5 & 93.3 & 7.7 & 41.8 & 93.3 & 7.7 & 41.8 & 93.9 & 8.1 & 43.3 & 88.6 & 5.9 & 41.2 \\
\hline 6 & 93.3 & 6.6 & 36.9 & 93.5 & 6.5 & 36.9 & 93.9 & 6.8 & 38.0 & 88.1 & 5.1 & 35.6 \\
\hline 7 & 93.9 & 5.7 & 35.2 & 93.9 & 5.7 & 35.2 & 94.5 & 5.9 & 36.2 & 88.1 & 4.4 & 33.9 \\
\hline 8 & 93.6 & 5.3 & 33.7 & 93.9 & 5.3 & 33.7 & 94.0 & 5.4 & 35.0 & 87.7 & 4.1 & 32.1 \\
\hline 9 & 93.7 & 4.8 & 30.5 & 93.7 & 4.8 & 30.5 & 94.3 & 5.0 & 31.5 & 87.5 & 3.8 & 28.9 \\
\hline 10 & 93.8 & 4.5 & 28.8 & 93.7 & 4.5 & 28.8 & 94.3 & 4.6 & 29.7 & 86.7 & 3.5 & 27.4 \\
\hline 11 & 93.4 & 4.3 & 27.0 & 93.5 & 4.3 & 27.0 & 93.7 & 4.4 & 27.6 & 86.1 & 3.3 & 25.5 \\
\hline 12 & 93.3 & 4.0 & 26.8 & 93.5 & 4.0 & 26.8 & 93.7 & 4.1 & 27.7 & 85.9 & 3.1 & 25.3 \\
\hline 13 & 92.9 & 3.8 & 24.4 & 93.3 & 3.8 & 24.5 & 93.7 & 3.9 & 25.3 & 85.4 & 3.0 & 23.2 \\
\hline 14 & 93.0 & 3.7 & 23.9 & 93.1 & 3.7 & 23.9 & 93.4 & 3.8 & 24.8 & 85.1 & 2.9 & 22.5 \\
\hline 15 & 92.7 & 3.5 & 23.3 & 92.8 & 3.5 & 23.3 & 93.0 & 3.6 & 24.1 & 86.8 & 2.7 & 21.9 \\
\hline 20 & 93.5 & 3.0 & 20.2 & 93.5 & 3.0 & 20.2 & 93.7 & 3.0 & 20.9 & 86.7 & 2.3 & 18.8 \\
\hline 25 & 94.4 & 2.6 & 17.7 & 94.5 & 2.6 & 17.7 & 94.6 & 2.7 & 18.3 & 87.0 & 2.0 & 16.5 \\
\hline 30 & 93.7 & 2.4 & 17.0 & 93.7 & 2.4 & 17.0 & 93.9 & 2.4 & 17.6 & 85.7 & 1.8 & 15.3 \\
\hline 35 & 94.7 & 2.2 & 15.6 & 94.7 & 2.2 & 15.6 & 94.9 & 2.2 & 16.2 & 87.6 & 1.7 & 14.3 \\
\hline 40 & 94.1 & 2.0 & 14.7 & 94.2 & 2.0 & 14.7 & 94.3 & 2.1 & 15.2 & 88.1 & 1.6 & 13.4 \\
\hline 45 & 95.0 & 1.9 & 13.7 & 95.1 & 1.9 & 13.7 & 95.3 & 2.0 & 14.3 & 88.0 & 1.5 & 12.9 \\
\hline 50 & 95.1 & 1.8 & 13.1 & 95.1 & 1.8 & 13.1 & 95.3 & 1.8 & 13.6 & 87.9 & 1.4 & 11.8 \\
\hline
\end{tabular}

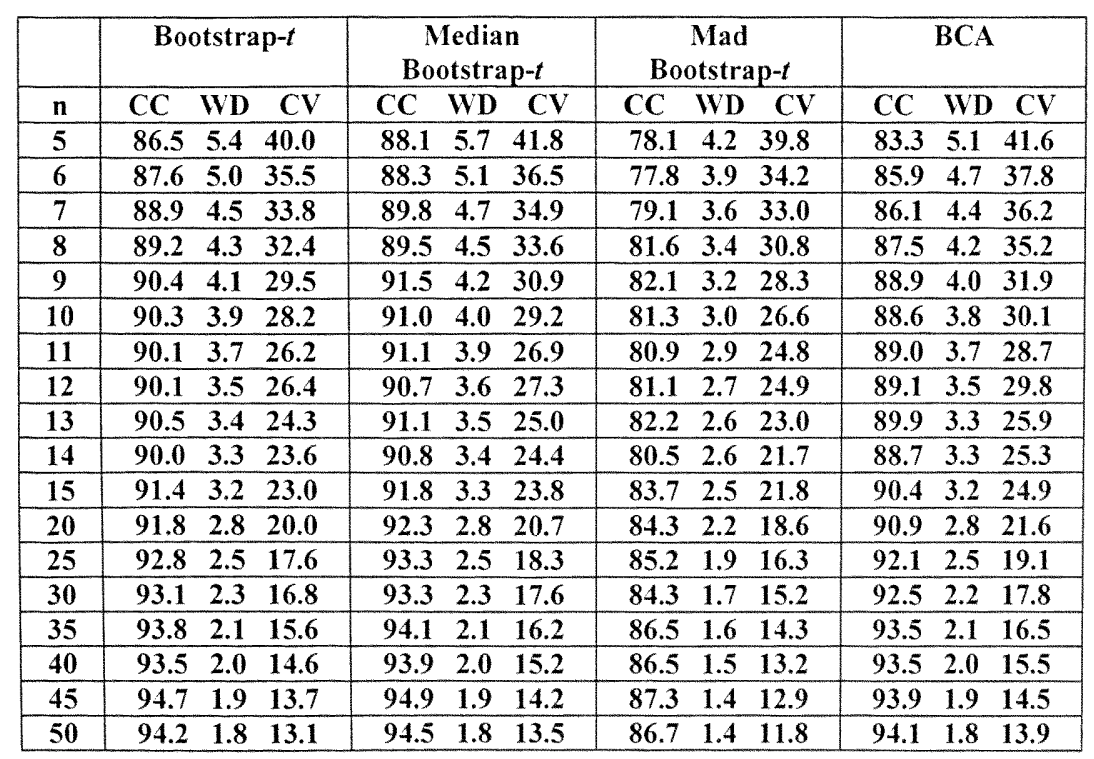




\begin{tabular}{|c|c|c|c|c|c|c|c|c|c|c|c|c|c|c|c|c|c|}
\hline \multirow[b]{2}{*}{$\mathrm{n}$} & \multicolumn{3}{|c|}{ T1 } & \multicolumn{3}{|c|}{ Median T1 } & \multicolumn{3}{|c|}{ Mad T1 } & \multicolumn{3}{|c|}{$\mathrm{T3}$} & \multicolumn{3}{|c|}{ Median T3 } & \multicolumn{2}{|c|}{ Mad T3 } \\
\hline & $\mathrm{CC}$ & WD & $\mathrm{CV}$ & $\mathrm{CC}$ & WD & $\mathrm{CV}$ & $\mathrm{CC}$ & WD & $\mathrm{CV}$ & $\mathrm{CC}$ & WD & $\mathrm{CV}$ & $\mathrm{CC}$ & WD & $\mathrm{CV}$ & $\mathrm{CC} W \mathrm{WD}$ & $\mathrm{CV}$ \\
\hline 5 & 88.7 & 8.1 & 63.5 & 89.5 & 8.6 & 65.3 & 81.1 & 6.1 & 59.8 & 90.3 & 8.1 & 43.6 & 91.3 & 8.6 & 45.3 & $81.9 \quad 6.1$ & 42.7 \\
\hline 6 & 88.3 & 7.0 & 62.6 & 89.0 & 7.3 & 64.4 & 79.9 & 5.3 & 56.1 & 89.5 & 7.9 & 39.0 & 90.1 & 8.2 & 40.3 & $81.5 \quad 5.9$ & 36.6 \\
\hline 7 & 89.3 & 6.0 & 62.6 & 90.1 & 6.3 & 63.9 & 80.3 & 4.6 & 55.0 & 91.0 & 7.6 & 37.2 & 91.5 & 7.9 & 38.2 & $84.5 \quad 5.7$ & 35.4 \\
\hline 8 & 89.7 & 5.7 & 61.0 & 90.5 & 5.9 & 63.5 & 82.9 & 4.3 & 52.6 & 91.5 & 7.5 & 35.2 & 91.9 & 7.8 & 36,4 & $85.8 \quad 5.7$ & 33.4 \\
\hline 9 & 91.1 & 5.1 & 58.0 & 92.0 & 5.3 & 60.0 & 83.9 & 3.8 & 49.3 & 91.3 & 7.4 & 31.9 & 91.9 & 7.7 & 32.8 & $84.1 \quad 5.6$ & 30.8 \\
\hline 10 & 91.3 & 4.8 & 55.9 & 91.9 & 4.9 & 57.5 & 83.3 & 3.7 & 47.4 & 92.4 & 7.3 & 30.4 & 93.0 & 7.5 & 31.4 & $86.7 \quad 5.5$ & 29.0 \\
\hline 11 & 91.1 & 4.6 & 53.8 & 91.5 & 4.7 & 54.7 & 81.6 & 3.5 & 45.0 & 91.1 & 7.2 & 28.2 & 91.7 & 7.4 & 29.0 & $85.3 \quad 5.5$ & 26.8 \\
\hline 12 & 91.0 & 4.2 & 53.6 & 91.7 & 4.3 & 54.7 & 82.7 & 3.2 & 43.9 & 93.5 & 7.0 & 28.2 & 93.7 & 7.2 & 29.1 & $88.7 \quad 5.3$ & 26.8 \\
\hline 13 & 91.2 & 3.9 & 49.0 & 91.7 & 4.0 & 49.6 & 83.3 & 3.0 & 41.3 & 92.7 & 6.8 & 25.8 & 93.2 & 7.0 & 26.5 & $\begin{array}{ll}87.5 & 5.2\end{array}$ & 25.3 \\
\hline 14 & 89.9 & 3.8 & 46.9 & 90.5 & 3.9 & 48.4 & 81.3 & 2.9 & 37.1 & 92.1 & 6.8 & 25.3 & 92.3 & 7.0 & 26.0 & $86.9 \quad 5.2$ & 24.0 \\
\hline 15 & 91.6 & 3.6 & 46.2 & 92.0 & 3.7 & 46.7 & 84.6 & 2.8 & 37.8 & 93.8 & 6.7 & 25.0 & 94.4 & 6.9 & 25.7 & $\begin{array}{lll}88.5 & 5.1 \\
\end{array}$ & 24.3 \\
\hline 20 & 92.2 & 3.0 & 35.1 & 92.4 & 3.1 & 35.6 & 84.3 & 2.3 & 28.0 & 93.5 & 6.3 & 21.4 & 93.8 & 6.4 & 22.1 & $\begin{array}{ll}88.3 & 4.8 \\
\end{array}$ & 19.9 \\
\hline 25 & 93.4 & 2.6 & 31.0 & 93.9 & 2.7 & 31.5 & 86.1 & 2.0 & 23.9 & 94.9 & 5.9 & 18.5 & 94.9 & 6.0 & 19.1 & $\begin{array}{lll}89.3 & 4.5 \\
\end{array}$ & 17.2 \\
\hline 30 & 93.7 & 2.3 & 24.0 & 94.1 & 2.4 & 24.5 & 85.3 & 1.8 & 17.9 & 95.9 & 5.3 & 17.7 & 96.1 & 5.4 & 18.4 & $\begin{array}{lll}90.7 & 4.1 \\
\end{array}$ & 16.2 \\
\hline 35 & 94.5 & 2.2 & 19.2 & 94.8 & 2.2 & 19.7 & 86.7 & 1.7 & 16.1 & 95.4 & 4.4 & 22.8 & 95.5 & 4.4 & 23.4 & $90.3 \quad 3.3$ & 20.4 \\
\hline 40 & 94.0 & 2.0 & 22.0 & 94.4 & 2.1 & 22.5 & 87.5 & 1.6 & 17.4 & 96.0 & 2.7 & 16.0 & 96.3 & 2.7 & 16.5 & $91.2 \quad 2.1$ & 14.2 \\
\hline 45 & 95.0 & 1.9 & 17.6 & 95.3 & 1.9 & 18.1 & 87.5 & 1.5 & 14.4 & 95.3 & 2.4 & 14.4 & 95.9 & 2.4 & 15.0 & $\begin{array}{lll}89.7 & 1.8 \\
\end{array}$ & 13.4 \\
\hline 50 & 94.6 & 1.8 & 17.7 & 94.8 & 1.8 & 18.2 & 86.3 & 1.4 & 13.8 & 96.3 & 2.2 & 13.8 & 96.5 & 2.2 & 14.3 & $89.8 \quad 1.7$ & 12.2 \\
\hline
\end{tabular}


Table A12: Estimated Coverage Probabilities using Log Normal $(1.96, .833)$ with Skewness $=4$ including Median and Mad Bootstrap $-t(\mathrm{CC}=$ Confidence Coefficient, $\mathrm{WD}=$ Average Width, $\mathrm{CV}=$ Coefficient of Variation)

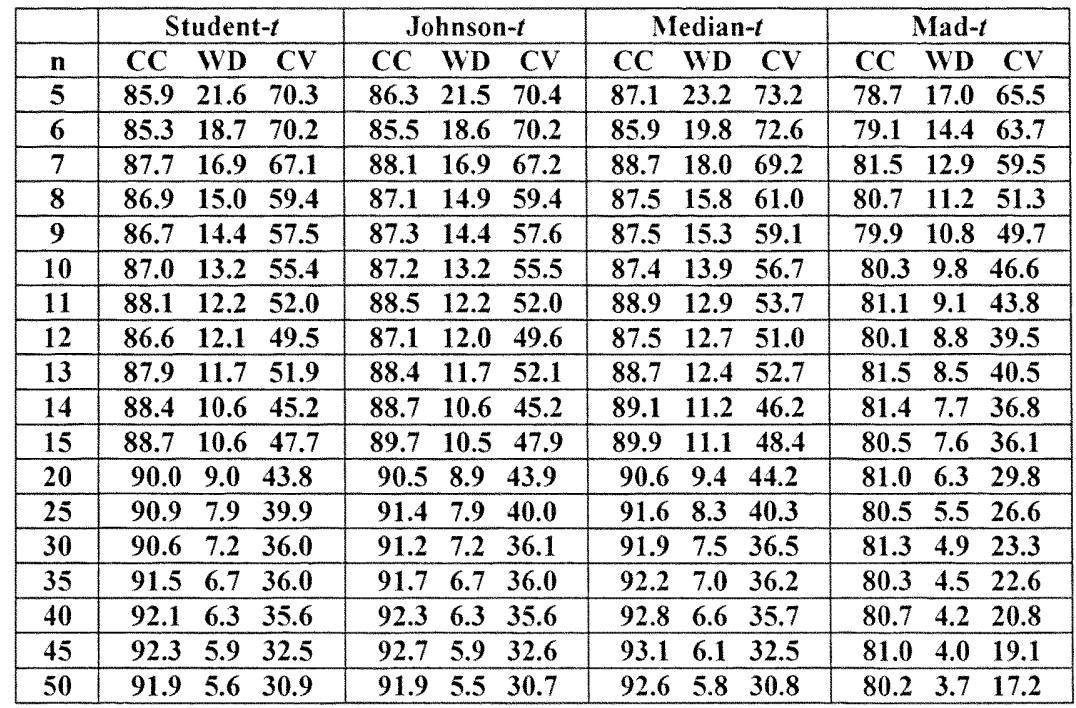

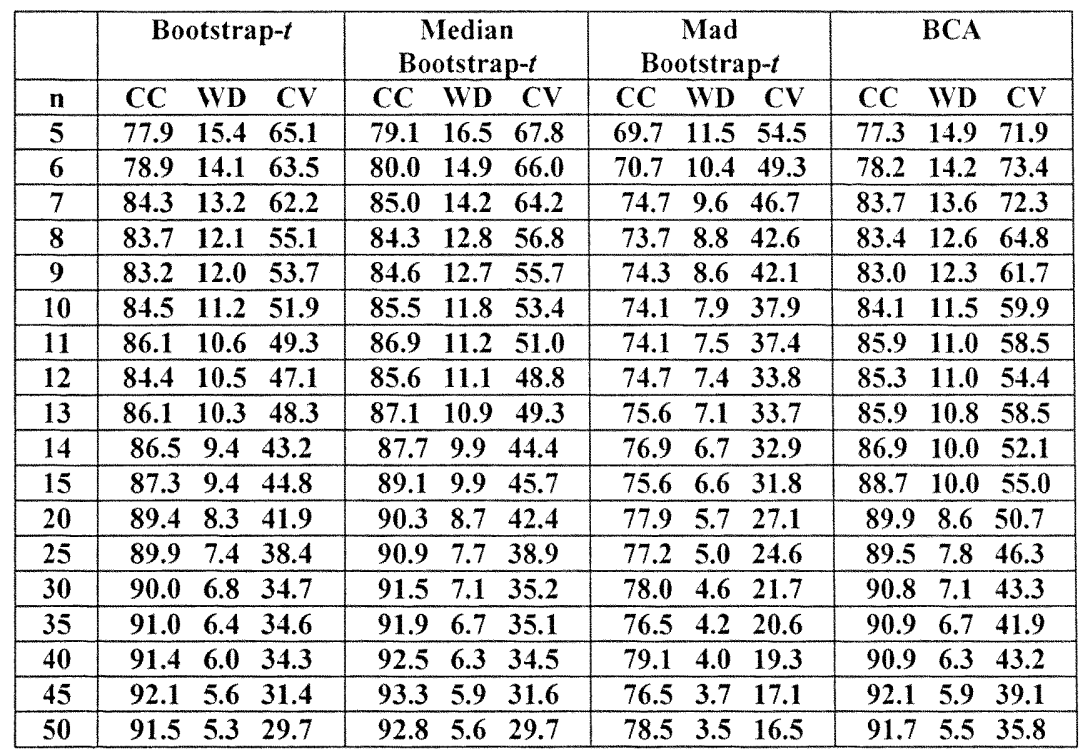




\begin{tabular}{|c|c|c|c|c|c|c|c|c|c|c|c|c|c|c|c|}
\hline & \multicolumn{3}{|c|}{$\mathrm{T} 1$} & \multicolumn{3}{|c|}{ Median T1 } & \multicolumn{2}{|c|}{ Mad T1 } & \multicolumn{3}{|c|}{ T3 } & \multicolumn{3}{|c|}{ Median T3 } & Mad T3 \\
\hline $\mathbf{n}$ & $\mathrm{CC}$ & WD & $\mathrm{CV}$ & $\mathrm{CC}$ & WD & $\mathrm{CV}$ & CC WD & $\mathrm{CV}$ & $\mathrm{CC}$ & WD & $\mathrm{CV}$ & $\mathrm{CC}$ & WD & $\mathrm{CV}$ & $\mathrm{CC}$ WD $\mathrm{CV}$ \\
\hline 5 & 84.4 & 25.9 & 87.3 & 85.5 & 28.0 & 90.5 & 76.918 .4 & 74.8 & 89.1 & 21.5 & 75.5 & 89.9 & 23.4 & 78.2 & $\begin{array}{llll}79.1 & 15.1 & 59.9\end{array}$ \\
\hline 6 & 4.5 & 24.1 & 87.9 & 85.0 & 25.7 & 90.4 & $76.7 \quad 17.1$ & 75.7 & 90.3 & 20.9 & 75.4 & 91.6 & 22.5 & 78.8 & $81.2 \quad 14.9 \quad 60.6$ \\
\hline 7 & 8.4 & 22.9 & 87.4 & 89.3 & 24.6 & 89.5 & $\begin{array}{ll}79.6 & 15.3\end{array}$ & 67.5 & 92.2 & 21.4 & 72.2 & 93.7 & 23.2 & 74.7 & $83.1 \quad 14.7 \quad 50.6$ \\
\hline 8 & 88.5 & 20.9 & 81.0 & 89.3 & 22.2 & 83.1 & $79.6 \quad 14.2$ & 66.4 & 93.7 & 20.3 & 64.5 & 94.5 & 21.6 & 66.1 & $\begin{array}{llll}84.3 & 13.8 & 46.9 \\
\end{array}$ \\
\hline 9 & 7.6 & 20.4 & 80.3 & 88.3 & 21.8 & 82.0 & $78.5 \quad 13.6$ & 65.3 & 93.2 & 20.4 & 61.9 & 94.7 & 22.1 & 64.3 & $\begin{array}{llll}44.1 & 13.9 & 46.6 \\
\end{array}$ \\
\hline 10 & 88.7 & 19.0 & 79.2 & 89.5 & 20.3 & 80.7 & $79.1 \quad 12.5$ & 61.7 & 95.1 & 19.8 & 59.9 & 96.0 & 21.1 & 62.0 & $86.6 \quad 13.5 \quad 43.5$ \\
\hline 11 & 90.1 & 17.8 & 77.5 & 91.3 & 19.0 & 79.8 & $79.0 \quad 11.6$ & 61.0 & 94.5 & 19.5 & 56.1 & 95.7 & 20.9 & 58.1 & $86.0 \quad 13.2 \quad 41.1$ \\
\hline 12 & 88.3 & 17.6 & 75.0 & 89.3 & 18.8 & 77.2 & $78.5 \quad 11.5$ & 59.8 & 95.2 & 19.7 & 52.8 & 96.2 & 21.0 & 55.2 & $\begin{array}{lllll}86.0 & 13.3 & 39.0 \\
\end{array}$ \\
\hline 13 & 88.9 & 17.0 & 76.8 & 90.3 & 18.1 & 78.2 & $78.5 \quad 11.0$ & 60.5 & 95.2 & 19.7 & 55.7 & 96.1 & 21.1 & 57.3 & $85.7 \quad 13.3 \quad 38.9$ \\
\hline 14 & 89.8 & 15.4 & 73.2 & 90.9 & 16.3 & 74.2 & $80.3 \quad 10.3$ & 57.7 & 95.5 & 18.7 & 49.4 & 96.4 & 19.9 & 50.9 & $\begin{array}{llll}87.7 & 12.8 & 36.3 \\
\end{array}$ \\
\hline 15 & 91.9 & 15.5 & 74.5 & 92.6 & 16.4 & 74.8 & $80.6 \quad 10.0$ & 57.3 & 95.9 & 19.1 & 51.1 & 97.2 & 20.4 & 52.4 & $\begin{array}{llll}87.6 & 12.8 & 36.7\end{array}$ \\
\hline 20 & 92.6 & 13.1 & 74.7 & 93.4 & 13.9 & 74.8 & $81.4 \quad 8.3$ & 54.0 & 97.3 & 18.2 & 47.0 & 97.7 & 19.3 & 47.5 & $\begin{array}{llll}89.2 & 12.0 & 30.7\end{array}$ \\
\hline 25 & 91.3 & 11.4 & 72.3 & 92.5 & 12.0 & 72.6 & $80.5 \quad 7.1$ & 51.5 & 95.7 & 17.0 & 42.7 & 96.9 & 18.0 & 43.5 & $86.7 \quad 11.2 \quad 27.3$ \\
\hline 30 & 92.3 & 10.0 & 70.8 & 93.3 & 10.5 & 70.7 & $81.1 \quad 6.3$ & 49.2 & 96.7 & 15.9 & 39.3 & 97.3 & 16.8 & 39.7 & $\begin{array}{llll}88.9 & 10.5 & 25.3 \\
\end{array}$ \\
\hline 35 & 92.9 & 9.2 & 71.5 & 94.1 & 9.6 & 71.3 & $78.4 \quad 5.6$ & 46.2 & 96.2 & 14.3 & 41.2 & 97.1 & 15.1 & 41.4 & $\begin{array}{llll}86.1 & 9.2 & 24.5 \\
\end{array}$ \\
\hline 40 & 92.8 & 8.3 & 71.6 & 94.0 & 8.8 & 71.4 & $81.2 \quad 5.2$ & 45.3 & 96.1 & 8.5 & 43.2 & 96.9 & 8.9 & 43.0 & $86.1 \quad 5.5$ \\
\hline 45 & 93.7 & 7.7 & 68.4 & 94.8 & 8.1 & 67.9 & $\begin{array}{lll}78.8 & 4.7 \\
\end{array}$ & 43.0 & 95.7 & 7.3 & 35.9 & 96.5 & 7.7 & 35.9 & $82.4 \quad 4.7$ \\
\hline 50 & 92.7 & 7.1 & 65.7 & 93.7 & 7.4 & 65.7 & 80.0 & 42.3 & 95.1 & 6.6 & 33.4 & 95.9 & 6.9 & 33.4 & $\begin{array}{llll}83.5 & 4.3 & 18.0 \\
\end{array}$ \\
\hline
\end{tabular}

